\title{
Functional Anatomy of Macaque Striate Cortex. II. Retinotopic Organization
}

\author{
Roger B. H. Tootell, ${ }^{1}$ Eugene Switkes, ${ }^{2}$ Martin S. Silverman, ${ }^{3, a}$ and Susan L. Hamilton ${ }^{1}$ \\ 'Department of Psychology, University of California, Berkeley, California 94720, ${ }^{2}$ Committee on Psychobiology, Division of \\ Natural Sciences, University of California, Santa Cruz, California 95064, and ${ }^{3}$ Department of Physiology, University of \\ California, San Francisco, California 94143
}

\begin{abstract}
Macaque monkeys were shown retinotopically-specific visual stimuli during ${ }^{14} \mathrm{C}$-2-deoxy-d-glucose (DG) infusion in a study of the retinotopic organization of primary visual cortex (V1). In the central half of $\mathrm{V}_{1}$, the cortical magnification was found to be greater along the vertical than along the horizontal meridian, and overall magnification factors appeared to be scaled proportionate to brain size across different species. The cortical magnification factor (CMF) was found to reach a maximum of about $15 \mathrm{~mm} / \mathrm{deg}$ at the representation of the fovea, at a point of acute curvature in the V1V2 border. We find neither a duplication nor an overrepresentation of the vertical meridian. The magnification factor did not appear to be doubled in a direction perpendicular to the ocular dominance strips; it may not be increased at all. The DG borders in parvorecipient layer $4 \mathrm{Cb}$ were found to be as sharp as $140 \mu \mathrm{m}$ (half-amplitude, half width), corresponding to a visual angle of less than 2 ' of arc at the eccentricity measured. In other layers (including magnorecipient layer $4 \mathrm{Ca}$ ), the retinotopic borders are broader. The retinotopic spread of activity is greater when produced by a low-spatial-frequency grating than when produced by a high-spatial-frequency grating. Orientation-specific stimuli produced a pattern of activation that spread further than 1 $\mathrm{mm}$ across cortex in some layers. Some DG evidence suggests that the spread of functional activity is greater near the foveal representation than near $5^{\circ}$ eccentricity.
\end{abstract}

The striate cortex is the locus of a major reorganization of visual information. It is here that a center-surround representation from each of the 2 retinal images undergoes a significant reorganization in terms of a more specialized coding (e.g., along the dimensions of orientation, periodicity, direction, binocularity, etc.). Presumably these latter receptive-field specializations provide a basis for even greater abstraction at higher visual centers. Thus, an understanding of the striate retinotopic map and its

\footnotetext{
Received July 2, 1986; revised Sept. 10, 1987; accepted Sept. 10, 1987.

We thank Russell L. De Valois for his generosity in supporting this long series of experiments. This work was supported by United States Public Health Service Grants EY-00014 and EY-02050 and National Science Foundation Grants BNS 82-02275 and BNS 78-86171.

Correspondence should be addressed to Roger B. Tootell at his present address: Department of Neurobiology, Harvard Medical School, 25 Shattuck St., Boston, MA 02115.

a Present address: Central Institute for the Deaf, $818 \mathrm{~S}$. Euclid Ave., and the Department of Anatomy and Neurobiology, Washington University, St. Louis, MO 63310.

Copyright (C) 1988 Society for Neuroscience $0270-6474 / 88 / 051531-38 \$ 02.00 / 0$
}

relationship to topographical feature maps (e.g., orientation columns, etc.) is of considerable interest.

Retinotopic organization has typically been measured by making multiple electrophysiological recordings (Talbot and Marshall, 1941; Daniel and Whitteridge, 1961; Hubel and Wiesel, 1974; Guld and Bertulis, 1976; Dow et al., 1981; Van Essen et al., 1984). In this approach, receptive-field location in the visual field is plotted for a large number of recording sites within a given visual area. After histological reconstruction of the recording sites, the receptive-field position at each recording site is plotted in a summary diagram, and an idealized map is interpolated between sampled sites.

In the present study we have instead used the ${ }^{14} \mathrm{C}$-2-deoxy$d$-glucose (DG) technique to study the retinotopic organization. In this alternative approach, an animal views an appropriate stimulus, and DG is used to mark regions of brain that are activated by the stimulus. Although the electrophysiological mapping approach offers some on-line feedback that is lacking in the DG approach, the DG mapping approach offers some complementary advantages that make it possible to study aspects of the retinotopic organization that are very difficult to measure with electrophysiological techniques.

According to one recent electrophysiological report, "[DG mapping] is inherently more precise than physiological methods. .." (Van Essen et al., 1984). The increased precision of the DG technique arises from the fact that, with DG mapping, the complete map is produced in the tissue itself: large-scale interpolations between sampled points are not required (as in electrophysiological mapping) and this eliminates a major source of ambiguity. Along with these interpolation problems, electrophysiological mapping precision is hampered by ambiguities due to minor eye movements, uncertainties in the correspondence of various retinal landmarks to positions in the visual field, uncertainty about exact laminar position, etc. For a number of reasons, these problems are less of a problem with the DG mapping technique we describe here: DG stimulation times arc short cnough so that cyc drift is not a problcm, the exact alignment of the stimulus on the cortex can be demonstrated from the autoradiographic result, and the retinotopic maps can be easily discriminated within each of the various striate cortical laminae.

In a preliminary report, we briefly described aspects of the overall retinotopic organization in the central half of the striate cortex (Tootell et al., 1982a); here we present full details from that study. Included in the present account is a detailed discussion of the magnification factor at various eccentricities and along various meridia. We also examine the central half of striate 
cortex for the possibility of a relative underrepresentation of the superior visual field, as has been reported in peripheral striate cortex by Van Essen et al. (1984).

In addition to these global aspects of the retinotopic organization, we present data on the finer aspects of the retinotopic organization that are particularly amenable to study with the DG technique. For instance, we devote some effort to defining the position of (and the cortical magnification at) the foveal representation. Such an issue can be addressed directly with DG because the greater magnification in the foveal representation causes a characteristic distortion in the representation of visual stimuli in striate cortex.

Another subject that we address is the relationship between ocular dominance strips and retinotopic organization. One constraint on the striate retinotopic organization is that 2 complete maps of the visual field feed into this area, one into each set of ocular dominance strips (Hubel and Wiesel, 1974; Blasdel and Fitzpatrick, 1984). Within striate cortex, the information in these 2 monocular input maps may be combined to some extent into a single output map because most cells in the extragranular striate layers (and the great majority of cells in extrastriate cortex) respond to input from either eye. It has been suggested that the 2 monocular input maps are effectively "squeezed" together in striate cortex (presumably to obtain a singular retinotopic representation in the binocular layers) by halving the magnification factor perpendicular to the ocular dominance strips (Hubel et al., 1974; Hubel and Wiesel, 1977). In this study we attempt to resolve some of these issues concerning the ocular dominance-retinotopic interaction with a fine-grained analysis of the DG mapping patterns.

Wc also analyze the DG maps for evidence on the horizontal spread of activity within striate cortex. In electrophysiological studies, it has been reported that the receptive fields of cells in a given striate region overlap those of nearby cells within a distance of about $2 \mathrm{~mm}$ across cortex (Hubel et al., 1974; Hubel and Wiesel, 1977). That is, one would have to move a microelectrode at least $2 \mathrm{~mm}$ in a direction parallel to the cortical surface in order to record receptive fields that did not overlap those from the primary recording site. We refer to this as the "minimum non-overlap" distance. It is closely related to measures of the "cortical point image" (for review, see McIlwain, 1986), which describes the horizontal spread of activity in striate cortex from a single point of activation in the visual field.

Though the idea of a single distance for the minimum nonoverlap distance is appealing, a number of complications have arisen. For one thing, it is widely reported that the average receptivc-ficld size varies in different striate layers. To the extent that this is true, the horizontal distance between nonoverlapping receptive fields must likewise vary with laminae. Such a discrepancy between non-overlap distances (correlated with differences in receptive-field size) has been shown directly in electrophysiological comparisons of cells in layers $4 \mathrm{Ca}$ and $4 \mathrm{Cb}$ (Blasdel and Fitzpatrick, 1984). However, such a comparison is not easy to imagine across all striate layers, using electrophysiological techniques, because of the technical limitations described above. In the present report it proved trivial to quantify a DG measure of the minimum non-overlap distance in different layers by simply examining the borders of DG maps in each layer. The issue of laminar differences in horizontal spread is important to analyze, because each striate layer projects to (and receives input from) different visual areas, each with a different magnification factor and retinotopic resolution.
Even within a given layer, the minimum non-overlap distances must be very rough approximations. Receptive-field size at any one eccentricity varies widely (by a factor of about 10 in area; Van Essen et al., 1984), and a large proportion of this variability range can be seen within a given layer (e.g., Gilbert, 1977). A wide variability in receptive-field size within a given layer can also be assumed from the columnar component of spatial frequency organization (Tootell et al., 1981, 1982b, 1988d; Silverman, 1984) and from the wide range of preferred spatial frequencies found at a given eccentricity (Movshon et al., 1978; De Valois et al., 1982). Cells tuned to a high spatial frequency should have smaller receptive fields than do those tuned to a low spatial frequency, if we assume an equal number of subregions. Obviously, then, the minimum non-overlap distance will vary, depending on whether one is recording from low- or highspatial-frequency units (small or large receptive fields) within a given layer. Again, such an issue would be difficult to study with electrophysiological techniques, but proved easy to study by simply measuring the horizontal spread of DG uptake produced by gratings of high or low spatial frequency.

A similar complication arises in considering the use of orientation-specific activity. In cross-correlation studies, it has been reported that cells with common orientation sensitivities communicate over fairly large distances (Ts'o et al., 1983). So it is possible that the minimum non-overlap distance depends on the relative orientation preference of the cells in question. This was also an easy question to address by using DG to label the activity produced by a visual stimulus that was both retinotopically and orientation-specific.

As a final complication, it has been reported that the minimum non-overlap distance in striate cortex also varies with eccentricity in a distinctly nonlinear manner (Dow et al., 1981; Van Essen et al., 1984). The most prominent variation is a steep increase in the extent of overlap from a minimum near $5^{\circ}$ towards a maximum near the fovea. Such a claim is important to verify because the reported variations with eccentricity signal a striking discrepancy between the architecture underlying the minimum non-overlap distance and the architecture underlying the cytochrome oxidase blobs and functional hypercolumns: the latter vary only slightly with eccentricity (Livingstone and $\mathrm{Hu}$ bel, 1984a), while it has been reported that the former vary greatly (Dow et al., 1981; Van Essen et al., 1984).

At this juncture, some terminological issues should be clarified. According to some authors, the minimum non-overlap distance (cortical point image) is "the basis of the modular concept" (Livingstone and Hubel, 1984a). Other authors have pointed out that the cytochromc oxidase blobs and the functional hypercolumns in striate cortex furnish 2 alternative definitions of a striate cortical "module" (Horton, 1984; Van Essen et al., 1984). At this point in history, it would appear that a "module" has become a multidefined term, meaning different things to different people. Since, in this paper, we analyze a number of aspects of the retinotopic micro-organization (including "modules"), it is useful to clarify what we mean by the various terms.

The minimum non-overlap distance and cortical point image are measurements of the physiological spread of activation. As we understand it, a "hypercolumn" refers to a complete cycle of stimulus specificities across cortex, such as an orientation "hypercolumn" or an ocular dominance "hypercolumn" (e.g., Hubel and Wiesel, 1977). Hypercolumns for other stimulus parameters (such as color, spatial frequency, or direction) have 
also been suggested in striate cortex (e.g., Michael, 1981; Tootell et al., 1981). A cytochrome oxidase (cytox) "blob" (also known as a puff, a patch, or a spot) is an anatomical entity found prominently in the upper striate layers. Because it has been shown that the cytox blobs are intimately linked to so many aspects of the striate cortical architecture, we refer to one cytochrome oxidase blob plus half the surrounding interblob area as a "blob domain." It has been shown that the spacing and geometry of various hypercolumns are rigidly linked to the cytox blobs (Horton and Hubel, 1981; Tootell et al., 1982b; Horton, 1984), so the hypercolumns are probably related more closely to the dimensions of a blob domain than to measurements of physiological spread.

In a final set of analyses we relate (1) the sharpness of borders in the DG maps to the psychophysically measured acuity limits of the Old World primate; (2) the magnification factor seen in our DG maps to the distance between cytochrome oxidase blobs (a blob domain) in the same tissue, and (3) the sharpness of DG retinotopic borders to the reported size of single-unit receptive fields at the same eccentricity.

\section{Materials and Methods}

Surgical and histological details in this study are similar to those described in the accompanying studies (see Tootell et al., 1988a). Eight macaque monkeys ( $M$. arctoides, $M$. nemestrina, $M$. fascicularis, and $M$. assamensis) were used in experiments that were designed to directly assess retinotopic organization; these we refer to as "detailed" mapping cases (cases 9, 13, 18, 23, 26, 30, 44, 52). Some retinotopic information was also available in results from a larger number of monkeys (used in related DG studics) that wcre shown split-field stimuli. In these latter animals, the representation of the horizontal and vertical meridian was often distinguishable from surrounding DG patterns, and these DG borders also furnished some retinotopic information. In an even larger pool of cases, a circular mask was often used to delimit the stimulus; in these cases the representation of a single iso-eccentric line was clearly mapped across the edge of the operculum in the resultant autoradiographs. A summary of all experimental stimulation conditions can be found in Table 1 of the preceding paper (Tootell et al., 1988a).

In previous studies, retinotopic information has normally been derived from macaques of a single species. In the present study, macaque monkeys of 3 different species (and correspondingly different brain sizes) were used so that differences as well as similarities in "the" retinotopic maps could be assessed.

Expcriments were carried out on lightly anesthetized, pharmacologically paralyzed monkeys. In the direct retinotopic tests, doses of paralytic were kept high (20-25 mg/ $\mathrm{kg} / \mathrm{hr}$ gallamine triethiodide, $1-3 \mathrm{mg} /$ $\mathrm{kg} / \mathrm{hr}$ tubocurarine) in order to minimize residual eye movements. In most cases, the foveal projection was defined by mapping the receptive fields of foveal striate cells in microelectrode recordings and the eyes were centered on the stimulus screen accordingly. General stimulus parameters are described below, and specific stimulus details are given for each case in Results. The monkeys were injected with $12.5-50 \mu \mathrm{Ci} /$ $\mathrm{kg}$ DG while they viewed each stimulus pattern. In about half of the detailed retinotopic tests, a full cycle of each stimulus variation took place within a second or less. In these cases, DG was injected in a single bolus, rather than in protracted injections. In all of the detailed mapping cases, the animal was euthanized 25-30 (rather than 45) min after the end of the DG infusion in order to reduce the effects of residual cyc movements on the DG patterns. Subsequent histological processing was as described in the preceding paper (Tootell et al., 1988a).

Measurements of DG retinotopic borders were straightforward in the flattened sections, except when a portion of the map was buried in the ectocalcarine fissure. Usually, no attempt was made to recover distances in the DG maps across the fissure. Since the fissure is largely confined to the inferior half of the operculum, distances along some meridia (e.g., the representation of the lower vertical meridian) were ncver obscurcd by the fissure. For most quantitative analyses, optical densities in the autoradiographs were converted to relative levels of DG uptake by calibration against ${ }^{14} \mathrm{C}$ radiographic standards. Relative levels of uptake were usually measured in 1 -dimensional scans across a given retinotopic border within a single layer. Resolution along the long axis of the scans was $25 \mu \mathrm{m}$; densities perpendicular to this axis were usually sampled over a larger distance $(50-100 \mu \mathrm{m})$ in order to reduce noise.

In all but one of the detailed retinotopic tests, stimuli were presented monocularly in order to prevent double (diplopic) images in the resultant autoradiographs. The stimuli in all of the detailed retinotopic tests included 3-5 rings (separated by equal-log intervals) and 4-8 rays, converging at the foveal projection. In half of the cases, the ring-and-ray configuration was either an achromatic solid $(n=1)$ or checked $(n=3)$ pattern of $100 \%$ contrast, counterphased on a gray background at the mean luminance. In 4 other cases, the background pattern varied in orientation, spatial frequency, and/or color, and the ring-and-ray pattern was superimposed on this more complicated background as an unvarying gray of mean luminance equal to that of the background. Since the stimuli were presented on a relatively flat screen, a tangent correction was included in all calculations of stimulus eccentricity. The maximum angular subtense of stimuli used in this study was $22^{\circ}$, so that retinotopic data in the present study are available only from the representation of the central $11^{\circ}$ (or less) of each hemisphere.

Distortion controls. The lateral striate operculum (where almost all of our data were sampled) is fairly flat in vivo, and in modeling studies it has been shown that this area can (theoretically) be unfolded without much distortion, as if one were unfolding a cylinder (Merker and Schwartz, 1985). In order to assess the magnitude and type of distortion in our actual procedure, we made some more formal measurements. In 7 hemispheres from 4 animals, we made 4 marks in the surface of striate cortex in situ with an India ink pen. The marks were laid out in each operculum in a roughly triangular grid, with the apex of the triangle at the fovea and 3 marks along the medial edge of the operculum forming the base of the triangle. Distances between the marks in situ were carefully measured by using thread and a ruler. After flat-mounting, 4 very small fiducial holes were drilled through the India ink marks, perpendicular to the cortical surface. (These fiducial holes can be seen, for instance, in the 2 autoradiographs from different layers in Fig. 4.) Thus, distances across the cortical surface in situ could be compared with distances between corresponding points in the autoradiographs, after histological procedures were complete.

In many of the hemispheres, there was either a shrinkage or an expansion of distances from the fovea to the medial edge of the operculum. The average change in distance between any 2 points in each hemisphere amounted to $10.8 \%$ of the distances measured in situ. However, this distortion sometimes took the form of an expansion, and, at other times, a compression. Averaged across all hemispheres, the overall distortion was only about $1 \%$.

Although it has been suggested that distances along horizontal and vertical meridians might be distorted differently within a single animal (Schwartz, 1985), this is not borne out by our data. In each of the 7 hemispheres on which direct measurements were made, distortions along the vertical meridian were compared to the distortion along the horizontal meridian of the same hemisphere. The average of the absolute values of this difference between vertical and horizontal meridia was only $2.5 \%$, with a maximum of $6 \%$. These minor differences are close to the average error in measurement. Averaged across all 7 hemispheres, distortion along the horizontal meridian differed from that along the vertical by only $0.6 \%$, which is certainly not significant. Section-tosection variability in the distance between fiducial holes was essentially negligible when care was taken to reflatten the cryostat sections on the knife blade before apposition to coverslips. No effort was made to correct for the possibility of brain shrinkage due to perfusion with a mildly hypertonic solution, since during dissection the hrain appeared to completely fill the cranial cavity.

\section{Results}

\section{Overall retinotopic organization}

\section{General description}

In our first retinotopic experiments $(n=2)$, one eye was occluded, while the other eye was shown a pattern of black and white checks, in a ring-and-ray configuration. The checks were presented in counterphase (white going to black and vice versa) against a gray background of luminance equal to the time-averaged mean of the black and white checks. The length of each check (parallel to the rings and rays) was randomized around a 


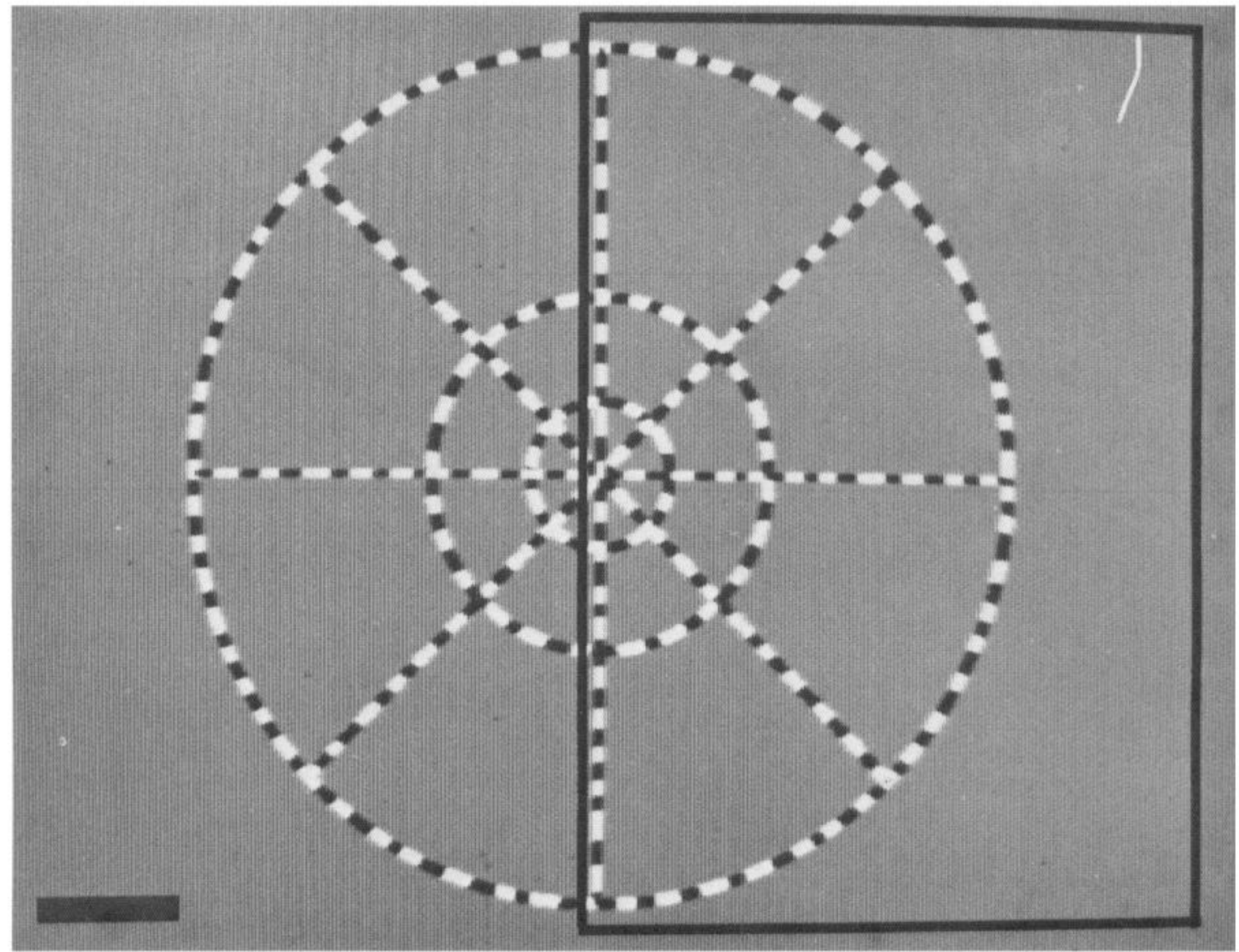

Figure 1. One of the stimuli used in the DG mapping study. During the actual experiment, the black and white checks reversed in contrast (that is, changed from black to white and back) in a temporal square wave at $3 \mathrm{~Hz}$. The luminance of the unvarying gray background was equal to the time-averaged mean luminance of the flickering black and white checks $\left(85.7 \mathrm{~cd} / \mathrm{m}^{2}\right)$. The stimulus was centered on the animal's fovea. The first ring is at $1^{\circ}$, the second ring at $2.3^{\circ}$, and the third at $5.4^{\circ}$. The solid black rectangle enclosing slightly more than half of the stimulus was not in fact part of the original stimulus; instead, it indicates that portion of the visual field projecting to one hemisphere (see Fig. 2). Calibration bar, $2^{\circ}$ in the visual field.

mean of $0.27^{\circ}$ (range, $0.18^{\circ}-0.4^{\circ}$ ), in order to minimize the possibility of selective uptake in a subpopulation of size-specific neurons. The checks were counterphased at $3 \mathrm{~Hz}$, which is near the average peak temporal tuning of striate cells.

In one case (13), the width of the ring-and-ray strips was kept constant $\left(0.13^{\circ}\right)$ across the stimulus screen. In this case, there were 3 rings (at $1^{\circ}, 2.3^{\circ}$, and $5.4^{\circ}$ ) and 8 rays (at $45^{\circ}$ intervals). This stimulus is shown in Figure 1. Two of the resultant autoradiographic sections are shown in Figure 2, $A, B$. Figure $2 A$ is taken almost entirely from layer 3 , and Figure $2 B$ is taken largely from layer 4 of the same hemisphere (reproduced from Tootell et al., 1982a). The correspondence of stimulus to its autoradiographic representation is diagrammed in Figure 3.

There was an initial concern that the retinotopic map might be confounded by uptake differences due to a higher metabolic activity in the blobs. In fact, there is a very faint dotted pattern in Figure $2 A$ (between the lines of intense, stimulus-specific activity) that is coextensive with the cytox blobs in stained sections (not shown). However, this cytox-related uptake is quantitatively so negligible that it can essentially be ignored (Tootell et al., 1982a; see also Tootell et al., 1988a).

From a comparison of Figure 2, $A$ and $B$, it is apparent that there is a certain amount of variation between layer 4 and layers $2+3$ in the resolution and contrast of DG results. This variation is accentuated in parafoveal regions, where the fixed size of stimulus checks is small relative to their representation in striate cortex. In parafoveal layer 4 , the small checks produce a crisp pattern of DG uptake, but in parafoveal layer 2, DG uptake is blurrier and low in contrast (see Fig. $2 A$ ). Differences between these and other laminae are described in full below.

The flickering black and white checks in the stimulus pattern produce a representation on striate cortex (see Fig. 2) that appears similarly "checkered." However, in this respect, the stimulus and autoradiograph are not actually isomorphic. The segmentation of the rays and rings in striate cortex is due to the monocular stimulation conditions. Because the visual field is duplicated within each set of ocular dominance columns, only half of the double-striate representation of a given retinal region was stimulated during the monocular stimulation conditions. The duplication of the visual field in each eye is illustrated quite graphically in a later section.

Although the width of the rings and rays was constant in the stimulus, their width in the DG map increases towards the foveal representation. This, of course, is due to variation in the striate magnification factor with eccentricity. By measuring the size of various aspects of the ring-and-ray representation, one can derive an exact measurement of the cortical magnification factor (CMF; mm cortex/deg visual angle). Measurements of 

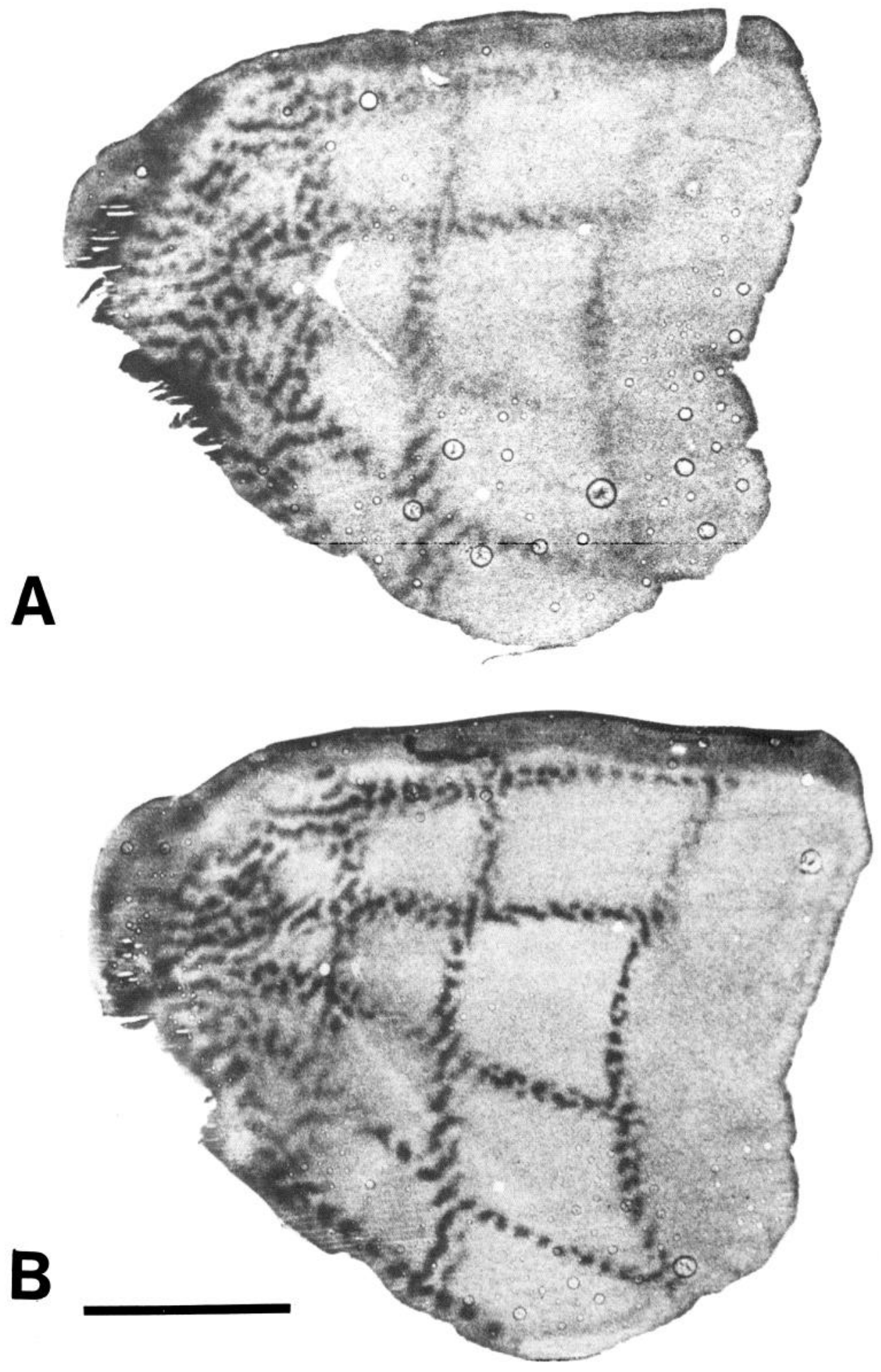

Figure 2. DG autoradiographs showing the effect of the retinotopic stimulus (Fig. 1) in various layers. The section in $A$ cuts through layers $2+3$, and the section in $B$ is mostly from layer $4 \mathrm{C}$. Both are tangential sections cut from the same flattened operculum, representing eccentricities from about $0^{\circ}-7^{\circ}$. The fovea is represented towards the left and the periphery is represented towards the right. The DG retinotopic borders are more sharply defined and higher in contrast in layer $4 C(B)$ than in the upper striate layers $(A)$, especially towards the periphery, where the cortical representation of stimulus checks becomes smaller. Scale bar, $1 \mathrm{~cm}$. the magnification factor from this and other cases are described in detail below.

\section{Duplication/overrepresentation of the vertical meridian}

In cat striate cortex, and in the retina of the cat and monkey, there is circumstantial evidence for a duplication of the vertical meridian (Stone et al., 1973; Kirk et al., 1976a, b; Bunt et al.,
1977; Tusa et al., 1978; Cusick et al., 1984). A "duplicated" vertical meridian would mean that areas of the visual field near the vertical meridian are represented twice in striate cortex, with one on each hemisphere near the V1-V2 border. In the absence of other mapping adjustments, this would mean that the striate cortical map would be expanded by a factor of 2 along the vertical meridian; i.e., there would be an overrepresentation of 

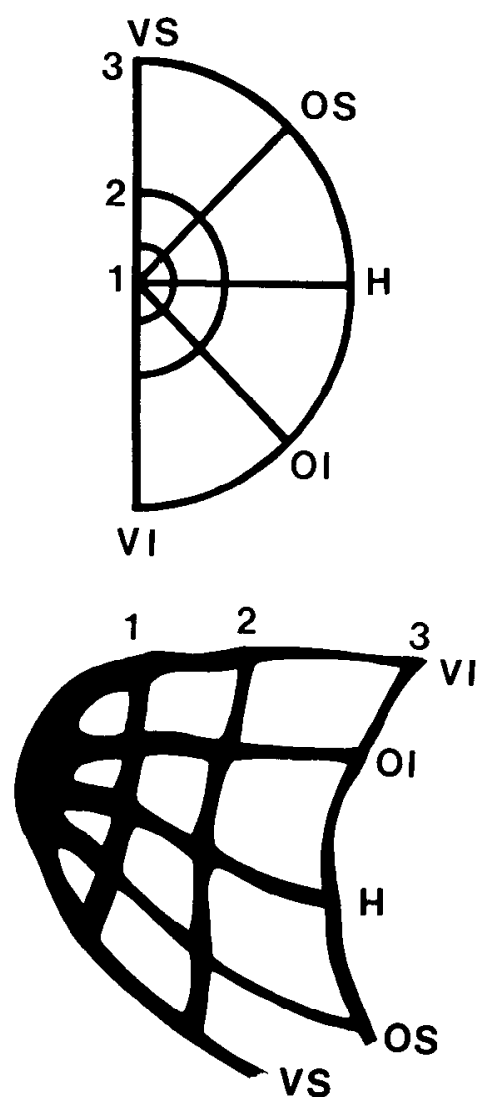

Figure 3. Schematic of the retinotopic transformation from stimulus (top) to striate cortex (bottom). 1, 2, 3, Selected regions from fovea to the periphery. VS and $V I$, vertical superior and vertical inferior rays, respectively. $O S$ and $O I$, oblique superior and oblique inferior rays, respectively. $H$, horizontal meridian.

the vertical meridian. Of course, it is possible to model a duplication of the vertical meridian without a corresponding overrepresentation. We first discuss evidence against an overrepresentation and then against a duplication.

In the DG case described above, the vertical stimulus rays were mapped onto the hemisphere shown in Figure 2, but they did not appear on the contralateral hemisphere. This indicates a slight experimental error in the horizontal positioning of the stimulus pattern relative to the midline, since if the stimulus strip were precisely centered, it should stimulate cortex in each hemisphere. If the vertical stimulus meridian had been positioned exactly on the stimulus center, it might be possible to resolve the question of whether there is an overrepresentation or duplication of this type within striate cortex, and, if so, the size of that effect.

In order to do this, we showed another monkey a quite similar stimulus, except that the stimulus was more carefully centered on the foveal representation in striate cortex (case 18). The stimulus in this case had 8 rays (separated by $45^{\circ}$ intervals) and 5 rings (set at eccentricities of $1.07^{\circ}, 1.90^{\circ}, 3.41^{\circ}, 6.08^{\circ}$, and $10.76^{\circ}$ ). The distances between rings were roughly equal on a logarithmic scale. In this case, the ring-and-ray widths varied with eccentricity: it was $0.15^{\circ}$ at the origin and over the first 3 rings, increased to $0.20^{\circ}$ at the fourth ring, and to $0.25^{\circ}$ at the fifth ring. The width of the rays was cqual to that of each ring at the ring-ray intersections, and varied linearly between rings.
Two autoradiographic sections from this case are shown in Figure 4 . Figure $4 A$ shows the DG results from layer 3 , and Figure $4 B$ is taken almost entirely from layer 4 . Since the peripheral rings have been increased in width relative to the previous case, there is no obvious falloff with eccentricity in the topography of upper-layer DG uptake (see Fig. 4A). In this case, about a quarter of the DG map in each hemisphere was obscured by a fold in the tissue that prevented full flattening of ventral striate cortex.

From the striate autoradiographic pattern is it is clear that the stimulus in this case was well-centered on the retina. In Figure 4, the DG representation of the vertical stimulus meridian extends to the very edge of the V1-V2 border at all eccentricities that could be examined. The V1-V2 border can be readily discerned in this and all of our autoradiographs by the presence of a sharp discontinuity in the level and pattern of DG uptake, especially adjacent to striate layer $4 \mathrm{C}$. In a number of cases, this break in autoradiographic uptake has in fact been confirmed as lying on the V1-V2 border, unlike sections stained for Nissl substance and cytochrome oxidase. For instance, Figure $5 C$ shows the $\mathrm{V} 1-\mathrm{V} 2$ border from the case illustrated in Figure 4 , as revealed by cytox staining of the section autoradiographed in $5 B$. Figure $5 A$ shows a portion of the vertical stimulus representation that appeared on the contralateral hemisphere. It is taken from an eccentricity identical to that of the position of the hemisphere with which it is apposed in Figure $5 B$, and it is shown at the same scale. On both hemispheres, the representation of the (bisected) vertical stimulus ray extends to the exact V1-V2 border.

If there were any overrepresentation of the vertical meridians in striate cortex, the sum of bisected vertical ray widths (Fig. 5, $A$ plus $B$ ) should be larger than the width of other DG ray segments that are mapped onto a single hemisphere (e.g., Fig. $5 F$ ), when comparisons are made at the same eccentricity. It is clear from Figure 5 that this is not the case. Within the limits of measurement, the sum of the bisected vertical ray segments is instead equal to that of the other ray segments at corresponding eccentricities.

It could be objected that a duplication of the vertical meridian remains possible without assuming a corresponding increase in the visual-field representation. For instance, let us suppose that along a narow (about $1^{\circ}$ wide) strip along the vertical meridian, only half of the LGN cells project to the "wrong" hemisphere, and half of the cells project to the correct hemisphere. Assume further that there are no bilateral projections. Such an idea is in fact consistent with the ganglion cell projections to the LGN (e.g., Bunt et al., 1977). Such a projection would naturally produce a duplication without an overrepresentation. However, it is contradicted by the DG data. In Figure 5, the width of the stimulus strip was $0.15^{\circ}$. This total width was shared between the right and left halves of the visual field, so that the total width on either side of the vertical meridian was about $0.1^{\circ}$ or less. In the ganglion cell data, the duplication in the projections arises from $1 / 2^{\circ}$ on each side of the vertical meridian (Stone et al., 1973; Bunt et al., 1977). Since the width of our vertical stimulus strip is much smaller than the presumptive width of the duplicated vertical meridian representation, there should be 2 representations of the vertical stimulus stripe, if there is, in fact, a duplication. Even if we assume that the width of the duplicated representation is severalfold smaller than $12^{\circ}$ on each side, one should see some evidence of a duplication distortion (for instance, a complete DG stripe representation on one side, and 


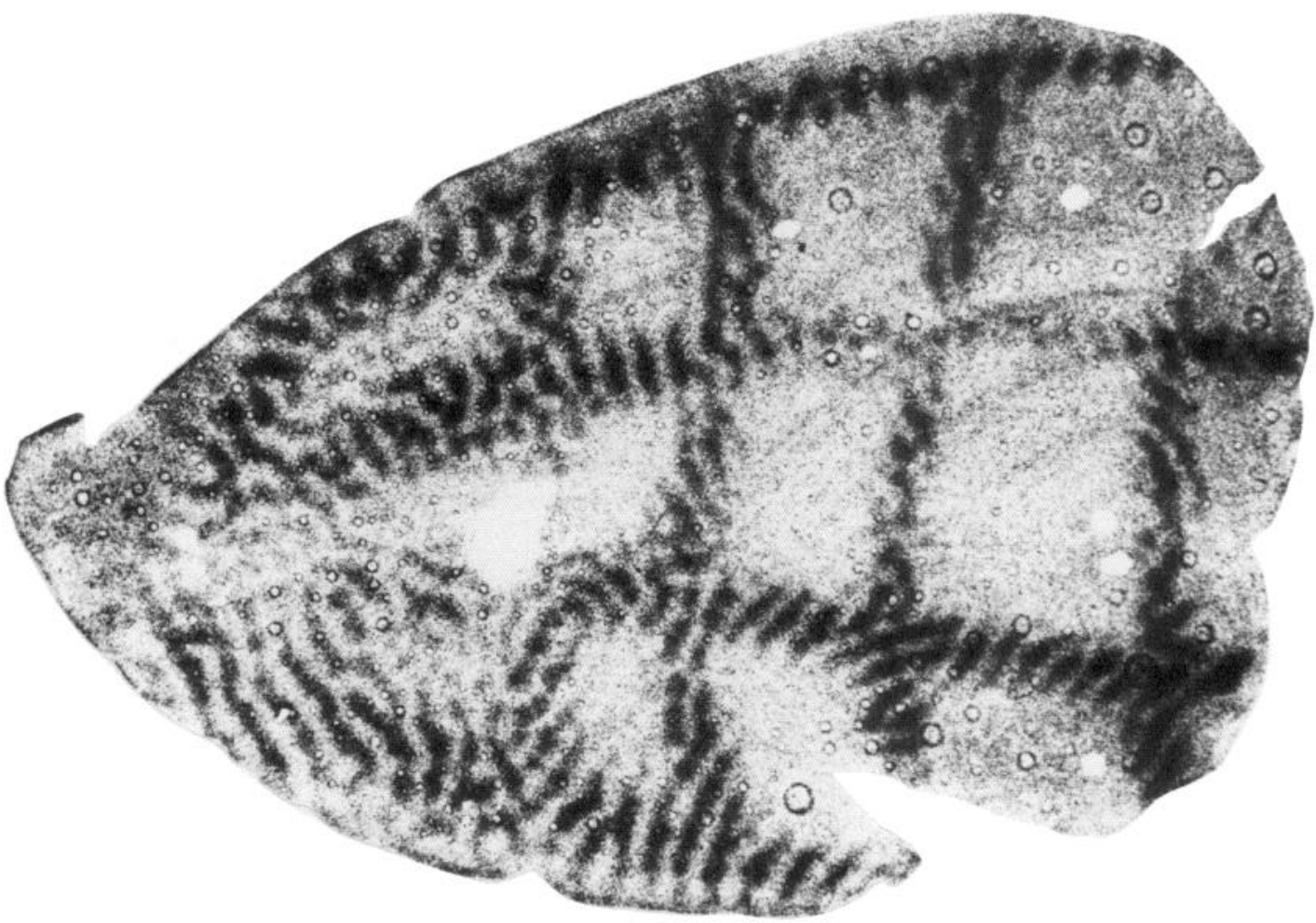

\section{A}

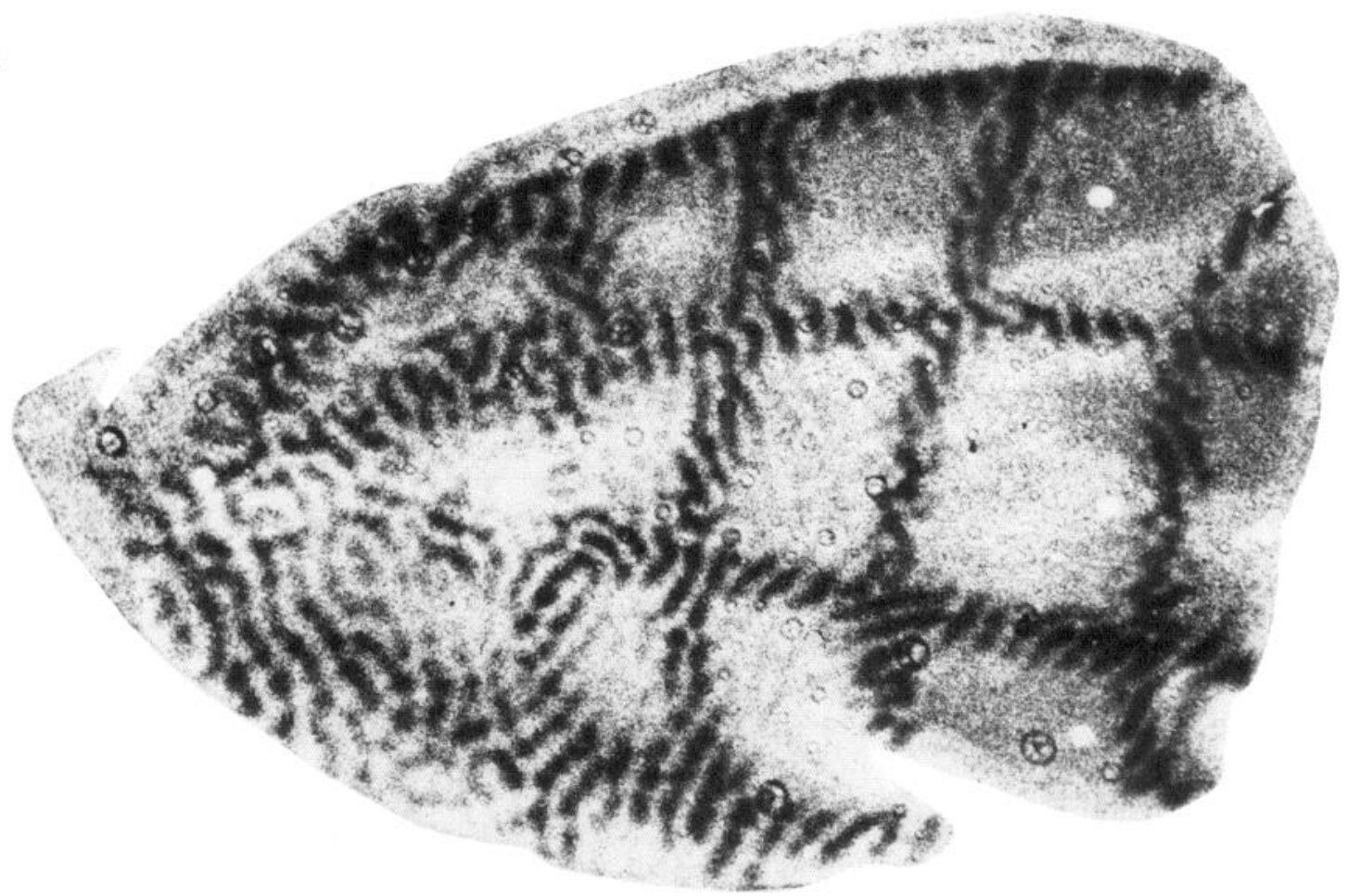

B

Figure 4. DG autoradiographs from a case similar to that described in Figures 1 and 2. As in Figure 2, both sections were cut tangentially from the same flattened operculum; $A$ is from layer 3 and $B$ is mostly from layer 4 . In this case, however, the stimulus had 5 rings, the width of the checks varied inversely with the cortical magnification, and the centering of the stimulus on the cortex was done more accurately. The fovea is represented to the left, and more peripheral striate regions to the right. Four fiducial holes can be seen by comparing $A$ and $B$. Towards the right of $A$ and $B, 3$ white spots can be seen in rough vertical alignment; these are tiny holes drilled perpendicular to the flattened cortical surface. The fourth fiducial hole appears towards the far left of both $A$ and $B$, very near to the foveal representation. The 4 fiducial holes are landmarks used to calculate the amount of distortion found in the flattening procedure and the amount of distortion obtained between successive sections (see Materials and Methods). Scale bar, $5 \mathrm{~mm}$. 


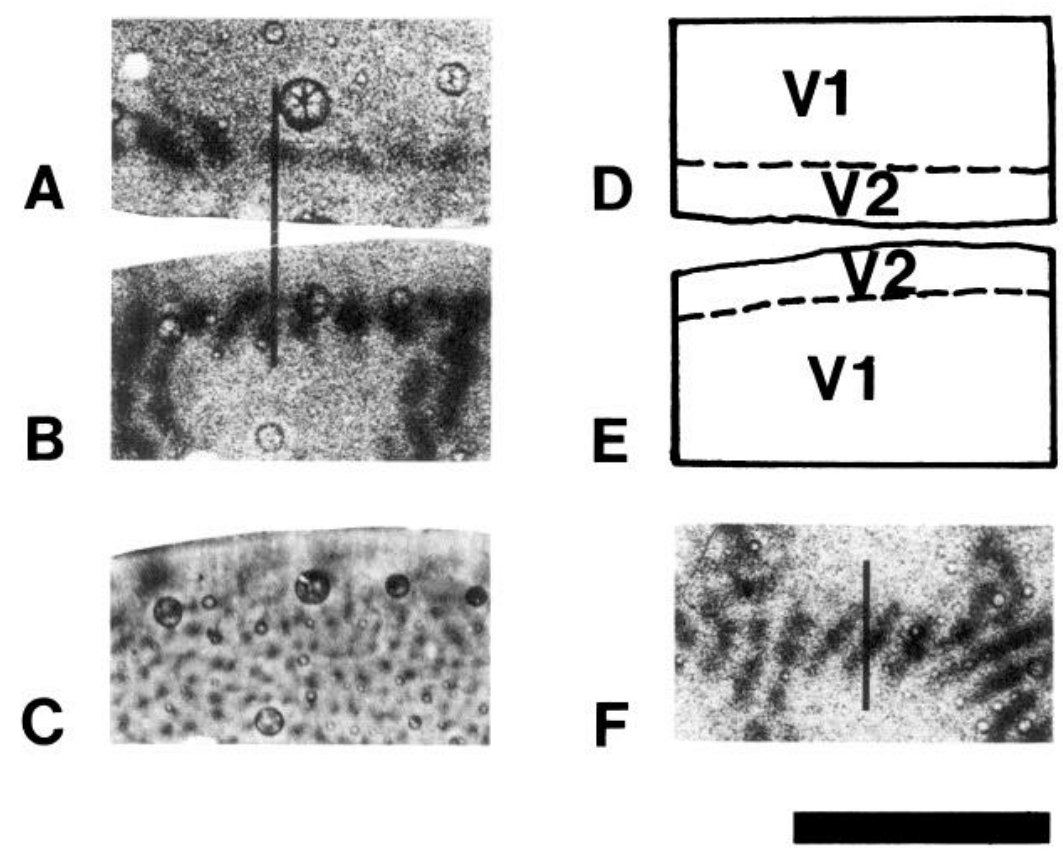

Figure 5. DG evidence against an overrepresentation or a duplication of the vertical meridian in striate cortex. $A-F$ are taken from the case illustrated in Figure 4. $F$, The autoradiographic representation of a stimulus ray running along the horizontal meridian. $A$, $B$, Representation of the corresponding stimulus ray running along the vertical meridian. The latter ray is of equal width and is displayed at the same eccentricities as the ray shown in $F$. $A, B$, The autoradiographic representation of the ray is shared between the 2 hemispheres. $B$ is taken from the same hemisphere as that shown in $F$, and $A$ is taken from the contralateral hemisphere. The shared autoradiographic representation along the vertical ray is divided along the V1-V2 border. The exact position of the V1-V2 border in $A$ and $B$ is shown in $D$ and $E$ (respectively), as judged from objective anatomical criteria; it shows the section used to produce the autoradiographs in $B$ after staining for cytochrome oxidase activity. The limits of the small circular cytochrome oxidase blobs in V1, and of the larger cytochrome oxidase strips in V2, allow the anatomical definition of the V1-V2 boundary in $E$. The position of the V1-V2 boundary in other sections (e.g., $D$ ) was judged on the basis of Nissl criteria; no discrepancies occur between the results from the 2 different staining techniques. If there was a duplicated representation of the vertical meridian (greater than $0.05^{\circ}$ ), a complete DG representation of this vertical stimulus ray would appear on both hemispheres. Instead, a single DG representation of the stimulus bar appears, split neatly in half along the V1-V2 borders of the 2 hemispheres. The thin, solid bar running roughly vertical in $A+B$ and $F$ indicates where measurements of the width of the ray representation should be made. The width of the stimulus-evoked DG uptake in $F$ is approximately equal to the combined width of the ring representation shared between the 2 hemispheres in $A+B$. If there were an overrepresentation of the vertical meridian in striate cortex, the combined width of the ray representation in $A+B$ should be significantly larger than that in $F$. Scale bar, 5 mm.

half of the same stripe representation on the other). No such distortions or duplicate representations are visible in the DG results.

\section{Representation and magnification of the fovea}

There has been some ambiguity as to the exact position of the striate foveal representation. At one point it was suggested that the fovea was represented almost a centimeter away from the V1-V2 border (Dow and Gouras, 1973). However, if there is no duplication of the vertical meridian, then the fovea must be represented on the V1-V2 border (see, for instance, Dow et al., 1985). In electrophysiological studies, it has usually been reported that the fovea is represented at or near a region of acute curvature (the "apogee") of the V1-V2 border, at the rostrolateral tip of the operculum. However, since the exact position of the foveal representation has not been fully resolved, and since the DG technique offers a precise measuring device, we examined a number of our cases for an answer to the question.

In 23 DG cases, we used stimuli with wedge-shaped sections having a common central point. In the 2 retinotopic cases we have described above, the stimulus was divided into 8 symmetric wedges; in other cases the stimulus was divided into 4 or 6 regions. In all 23 of these cases, we assumed that the fovea was represented at the rostrolateral apogee of the V1-V2 border, and we tried to position the center of the stimulus pattern accordingly. However, in some cases the stimulus center was inadvertently mapped some distance away from the intended region, and in these cases the ray-shaped borders would always veer away from the apogee (see Fig. $6, A-D$ ). In contrast, stimulus centers that were mapped exactly on the apogee were not distorted (see Fig. 5, $E-H$ ). The various distortions in the offcenter cases (and the lack of distortion in the on-center cases) strongly indicate that the horizontal meridian is mapped across the apogee of the V1-V2 border. Coupled with the evidence for a lack of duplication of the vertical meridian (in Fig. 5, as well as in other autoradiographs showing a lack of duplication through the apogee), it appears that the fovea is indeed mapped at the apogee of the V1-V2 border.

There are 2 other cases that bear on the issue of foveal mapping. In one case, we presented a horizontal, 0.5 cycle/deg squarewave grating monocularly (case 52 ). The grating was counterphased (that is, every black or white stripe changed to the reverse and back) at $3 \mathrm{~Hz}$, and it did not move across the stimulus screen (see Fig. 7A). The stimulus produced a DG result that clearly reflects the horizontal orientation of the stripes in the visual field.

We presume that the regions of high uptake in the DG pattern correspond to the representation of the visual stripe edges (rather 
than, for instance, to the center of the stripes) for several reasons. In other tests, it has been shown that striate regions stimulated with locally diffuse luminance variations (such as regions receiving input from the center of the wide stripes) take up very little stimulus-driven DG at all (Tootell and Silverman, 1981; see also the following paper Tootell et al., 1988b). Also, the periodic DG "strips" are fairly thin, as is the representation of the stripe edges; it is hard to imagine other portions of the stimulus stripes that would logically give rise to such a thin DG pattern. After all, only the edge of the stripes would give rise to an orientation-specific signal. Since in this experiment we used an extensive, repetitive stimulus pattern, no attempt was made to correlate a given stimulus stripe with a given isoclevation representation in the resultant DG pattern.

The retinotopic borders formed by the horizontal stripes are clearly visible across the operculum (see Fig. $7 B$ ). As in previous cases, the expansion of the regular stimulus pattern near the striate foveal representation reveals both the position of the foveal representation and the variation in cortical magnification with eccentricity. The maximal distance between adjacent stripes occurs at the V1-V2 border between stripes that straddle (although unevenly) the apogee: this is additional evidence that the fovea is represented at the apogee. The apparent horizontal meridian seems to be biased strongly towards the ventral half of the section in Figure 7. Though such a bias in the representation of upper and lower visual fields does occur in striate cortex (Van Essen et al., 1984; and see below), the bias in this case is exaggerated by illustrating a section that is missing small portions of ventral striate cortex.

We can also derive an estimate for the foveal cortical magnification factor from this case. The maximal distance between adjacent DG stripe borders at the foveal representation $(15 \mathrm{~mm}$, the average of 2 hemispheres) was produced by stripe borders $1^{\circ}$ apart in the visual field. Thus, the magnification factor at the foveal representation is at least $15 \mathrm{~mm} / \mathrm{deg}$. Of course, this must be an underestimate, because the "foveal" sample includes striate areas that are more than $1 / 2^{\circ}$ from the center of the foveal representation.

There is one final case that yields data on the size and position of the foveal representation (case 44). Here, a monkey was stimulated monocularly with a vertical stimulus strip that was $1.38^{\circ}$ wide, centered so that only half of the stimulus strip was mapped onto each hemisphere. Within the vertical strip, a black-white square-wave grating of variable orientation and spatial frequency was drifted in both directions at a wide range of speeds. Outside the stimulus strip, the background was mainly a steady gray, with the exception of a row of tiny blinking dots along the horizontal meridian and the 2 oblique rays (see Fig. $8 A$ ). The dots are irrelevant to the present discussion; they are described more fully below in the context of a different set of experiments. The time-averaged luminance was constant across the extent of the stimulus.

An autoradiograph from layer 4 of this case is shown in Figure $8 B$. In each hemisphere, half of the vertical stimulus strip is represented as a set of ocular dominance strips (owing to the monocular exposure) that end abruptly in a retinotopic (stimulus-induced) border running roughly parallel to the V1-V2 (anatomical) border. Apparently the stimulus was well-centered along the horizontal meridian, because the width of the visually stimulated region in the other hemisphere (at corresponding eccentricities) is virtually identical to that shown in Figure $8 B$. In both hemispheres, the width of the stimulated strip in striate

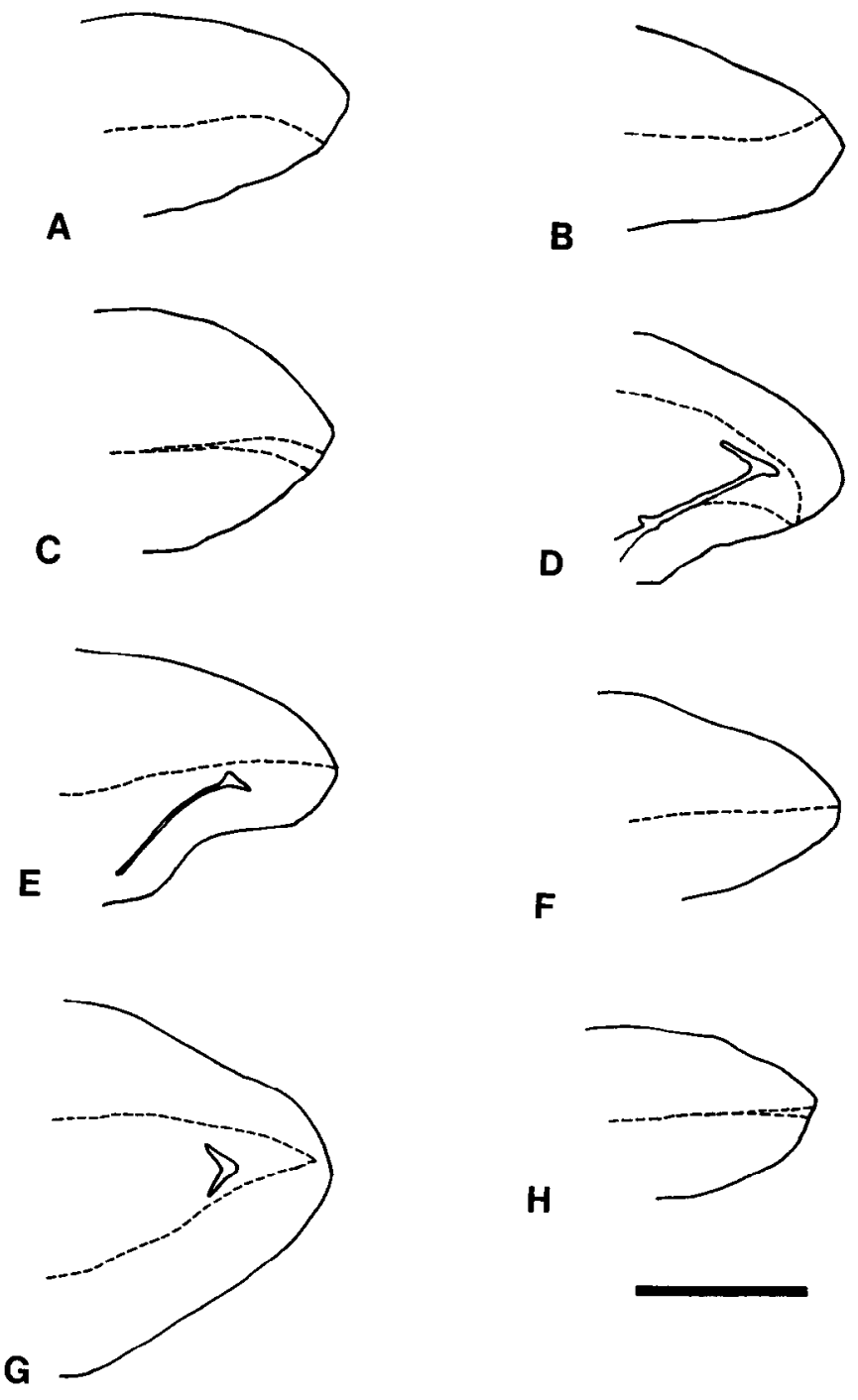

Figure 6. DG evidence that the fovea is represented in the region of acute curvature (apogee) at the V1-V2 border. In $A-H$, the region of acute curvature in the V1-V2 border (the presumptive foveal representation) appears on the right, and progressively more peripheral eccentricities are represented to the left. The outer solid line delimits the V1V2 border, except on the left, where it indicates the peripheral limit of $\mathrm{V} 1$, which can be seen on the flattened operculae. Where it appears, the solid line drawn inside V1 indicates an area of V1 cortex that did not flatten. The dashed lines indicate the DG retinotopic borders produced by various stimulus rays. Two figures $(C$ and $H$ ) were taken from binocular cases in which there was a representation of the horizontal meridian in each of the 2 (slightly misaligned) eyes; in these cases, the representation from each eye is indicated by dashed lines. $D, G$, From cases in which the stimulus contained 2 oblique rays in each hemisphere. Elsewhere, the dashed line indicates a monocular horizontal retinotopic border. In $A-D$, the stimulus rays were apparently positioned slightly above or below the horizontal meridian. Such a vertical misalignment produces a DG border that veers away from the region of acute curvature. In $E-H$, the stimulus was apparently well-centered vertically, so that the DG borders show no such distortion approaching the V1$\mathrm{V} 2$ border. The distortion in $A-D$ and the lack of distortion in $\mathrm{E}-\mathrm{H}$ indicate that the fovea is represented at the apogee of the V1-V2 border. Scale bar, $2 \mathrm{~cm}$.

cortex is greatest at the apogee, which supports the idea that the fovea is represented at the apogee. The minimum distance of the retinotopic border from the foveal representation is about $8 \mathrm{~mm}$ in each hemisphere. Since the stimulus was $0.7^{\circ}$ wide in 

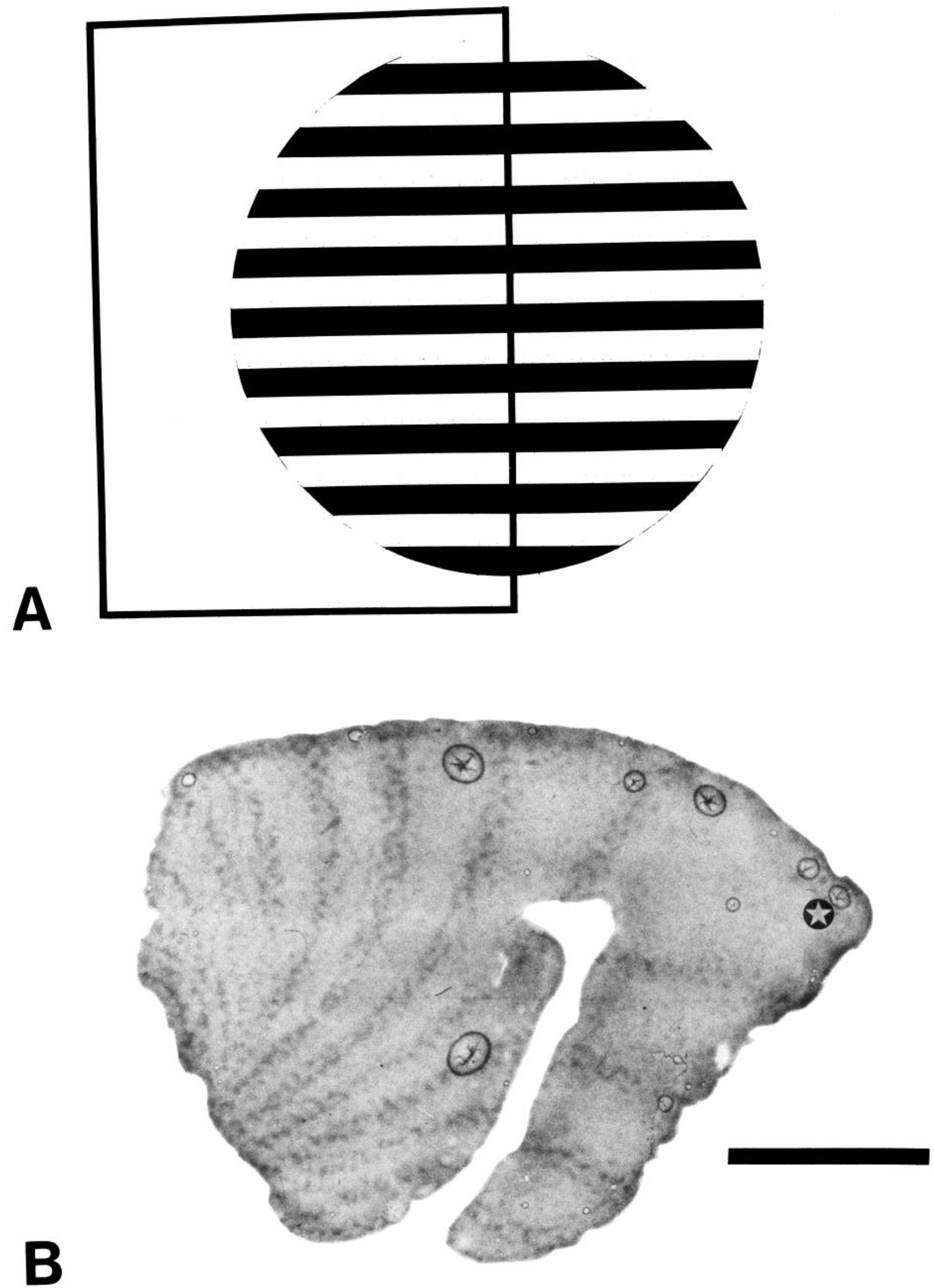

Figure 7. DG patterns produced by a stationary, counterphase flickering grating. The stimulus in this experiment $(A)$ was a black-white squarewave grating $(0.5 \mathrm{~cm} / \mathrm{deg})$ counterphased at $2 \mathrm{~Hz}$ and presented monocularly. That half of the stimulus projecting to one hemisphere is indicated by the solid black rectangle (which was not included in the original stimulus). $B$, The autoradiographic result of this stimulus in a single, tangential section through layer 3 from the flattened operculum to which the enclosed stimulus region projected. DG stripes (produced by the edges of the stimulus stripes) are clearly visible across the operculum. Again, horizontal retinotopic borders veer away from the foveal representation (star in 
each hemifield, this yields a foveal magnification factor of at least $11.6 \mathrm{~mm} / \mathrm{deg}$. This figure must again be regarded as an underestimate, since the cortical distance across which the measurement was taken extends some $7 \mathrm{~mm}$ from the point at which we wish to define the cortical magnification.

In the ring-and-ray case illustrated in Figures 2 and 4, we were able to measure the foveal cortical magnification factor more directly by measuring the width of the DG ray representation where the rays intersect the foveal representation. The measurement was most straightforward in the case of Figure 2, using the contralateral hemisphere (not illustrated). In this hemisphere, the foveal DG pattern was not obscured by the presence of the vertical ray due to the slight stimulus misalignment noted previously. The measurement was made on 2 oblique and 1 horizontal ray, at or near the apogee of the V1-V2 border. The average width of the 3 rays was about $2.2 \mathrm{~mm}$. Since the width of the stimulus was about $0.13^{\circ}$, this yields a foveal magnification factor of $16.9 \mathrm{~mm} / \mathrm{deg}$. From an analysis similar to that described above, our best estimate of the foveal CMF in the second ring-and-ray case (Fig. 4) is $14.7 \mathrm{~mm} / \mathrm{deg}$. If we compare the 2 values known to be underestimates $(11.6$ and $15 \mathrm{~mm} / \mathrm{deg})$ with the more direct estimates described above (14.7 and 16.9 $\mathrm{mm} / \mathrm{deg}$ ), we find fairly good agreement on a foveal magnification factor near $16 \mathrm{~mm} / \mathrm{deg}$. Since the DG maps reflect a certain spread of physiological activity (e.g., cortical point image; see below), it could be argued that our numbers on the foveal magnification factor may be overestimates, perhaps by as much as $10 \%$; this would bring our estimates down to $14-$ $15 \mathrm{~mm} / \mathrm{deg}$. Either figure is well within the range reported by most studies (e.g., Daniel and Whitteridge, 1961; Van Essen et al., 1984), hut is significantly smaller than that reported by Dow et al. (1985), and larger than the estimate of Hubel and Wiesel (1974).

Of our 4 estimates, the lower estimates from each category (11.6 and $14.7 \mathrm{~mm} / \mathrm{deg}$ ) were obtained from $M$. fascicularis, and the higher estimates ( 15 and $16.9 \mathrm{~mm} / \mathrm{deg}$ ) from the larger $M$. arctoides. Thus, there may be some species difference in the exact foveal magnification (see below for futher discussion).

\section{Extrafoveal cortical magnification factor}

In 4 cases $(13,18,23$, and 52), a stimulus containing 3-5 concentric rings was well-centered in foveal striate cortex. From the results of these cases it was possible to compute the CMF at various points across the extent of the lateral operculum, along a varicty of meridia. The cortical magnification at a given point on a meridian was calculated by dividing the cortical distance between adjacent rings (or between the first ring and the origin) by the distance between corresponding points in the stimulus. The resultant ratio (in $\mathrm{mm} / \mathrm{deg}$ ) was taken to be the $\mathrm{CMF}$ at an eccentricity midway between the eccentricity of the 2 sampled points. Of course, if the magnification factor increased in a roughly logarithmic manner towards the fovea, then the CMF estimated for the midway point would be a slight overestimate. However, we wished to avoid the a priori assumptions that this latter analysis would involve, and in direct tests com- pensation for this factor did not change the obtained magnifcation factors very much.

Along the representation of the lower vertical meridian (dorsal V1-V2 border), autoradiographic data from which the CMF could be calculated were available from 7 hemispheres in 4 animals. Because of the presence of a deep fissure across the operculum in one of the animals, data from the horizontal meridian were only available from 5 hemispheres ( 3 animals; cases $13,18,23)$. In an effort to correct for minor errors in centering the stimulus, data from the 2 hemispheres of the same animal were averaged together whenever possible.

These CMFs along the horizontal and inferior vertical meridia are plotted in Figure 9, $A$ and $B$, respectively. For convenience, the inverse of the CMF has been plotted rather than the CMF itself. Except near the fovea, the $\mathrm{CMF}^{-1}$ is fairly linear in each case. In Figure $9 A$, some variation can be seen in the functions between animals. Some of this variation is an artifact of interpolations in the CMF between widely separated data points: little corresponding variation is seen when cortical distance is plotted rather than local CMF (see below).

The average magnification factor along each ring has also been calculated, and these data appear as hollow triangles in Figure 10. The magnification factor along the rings is clustered closely around the mean for the horizontal meridian, and is clearly different from that measured along the vertical meridian. Presumably, then, this is further evidence for an anisotropic representation near the vertical meridian (see Van Essen et al., 1984).

In order to see if the CMF within a given area of striate cortex was relatively constant, we took photographs of autoradiographs from layer $4 C$ of 5 detailed retinotopic mapping cases, and cut out and wieghed those (roughly parallelogram-shaped) portions of the photographs bounded by each of the rings and rays. In comparisons of regions within a given iso-eccentricity strip, the areas were within about $10 \%$ of each other, and there were no consistent patterns in the differences that could be seen.

The average $\mathrm{CMFs}^{-1}$ along both the horizontal and inferior vertical meridia were computed from the data shown in Figure 9, and are compared in Figure 10. A best linear fit to the slope of the $\mathrm{CMF}^{-1}$ along the horizontal meridian is $0.108 \mathrm{deg} / \mathrm{mm}+$ $0.066 E / \mathrm{mm}$, where $E$ is eccentricity in degrees. A best fit to the vertical meridian is $0.070 \mathrm{deg} / \mathrm{mm}+0.052 E / \mathrm{mm}$.

At corresponding eccentricities, the $\mathrm{CMF}$ along the vertical meridian is larger than that along the horizontal by a factor of 1.25-1.5. Some relative increase along the vertical meridian is to be expected from purely geometrical considerations: in the unfolded schematic drawn by Daniel and Whitteridge (1961), the striate cortical representation of the vertical meridian appears to be physically longer than the horizontal by a factor of about 1.25. (Obviously, the exact factor will vary depending on the exact shape of striate cortex, which has some biological variability.)

An increased length along the vertical meridian (relative to the horizontal) is also illustrated in the very first mapping study of macaque striate cortex, by Talbot and Marshall (1941). In

$B$ ). More peripherally (to the left), the DG stripes appear to coalesce in accordance with a progressive decrease in the striate magnification factor. Estimates of the cortical magnification factor (at the fovea and more peripherally) can be easily derived by simply measuring the distance between the 2 stripes at selected loci. Scale bar, $1 \mathrm{~cm}$. 

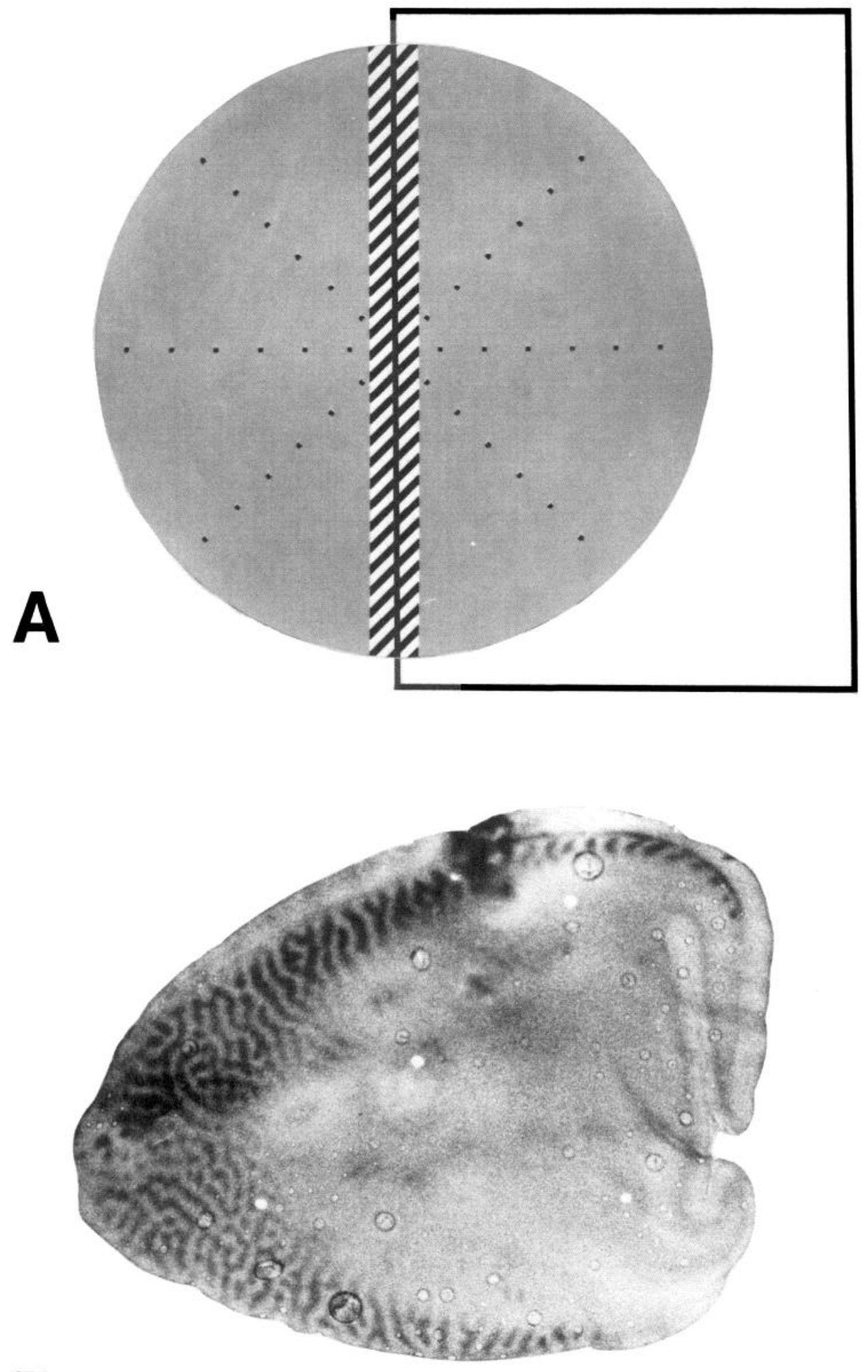

B

Figure 8. DG pattern produced by stimulation along a vertical strip. In this experiment, a grating of various orientations and spatial frequencies was presented monocularly within a thin $\left(0.7^{\circ}\right)$ region on both sides of the vertical meridian (see $A$ ). In addition to this, the stimulus contained a few blinking black and white dots, significant in the context of a manipulation described elsewhere. $B$, The autoradiographic result of the stimulation in and near upper layer 4 . The representation of the fovea is located to the left in $B$, and the periphery to the right. The DG representation expands near the fovea and contracts along the representation of the more peripheral vertical meridian. The striate magnification factor at various points along the vertical meridian can be calculated by measuring the width of the stimulus representation at selected loci. The striate representation of the blinking stimulus dots can also be seen faintly in $B$, especially along the representation of the horizontal and inferior visual fields. Scale bar, $1 \mathrm{~cm}$. 

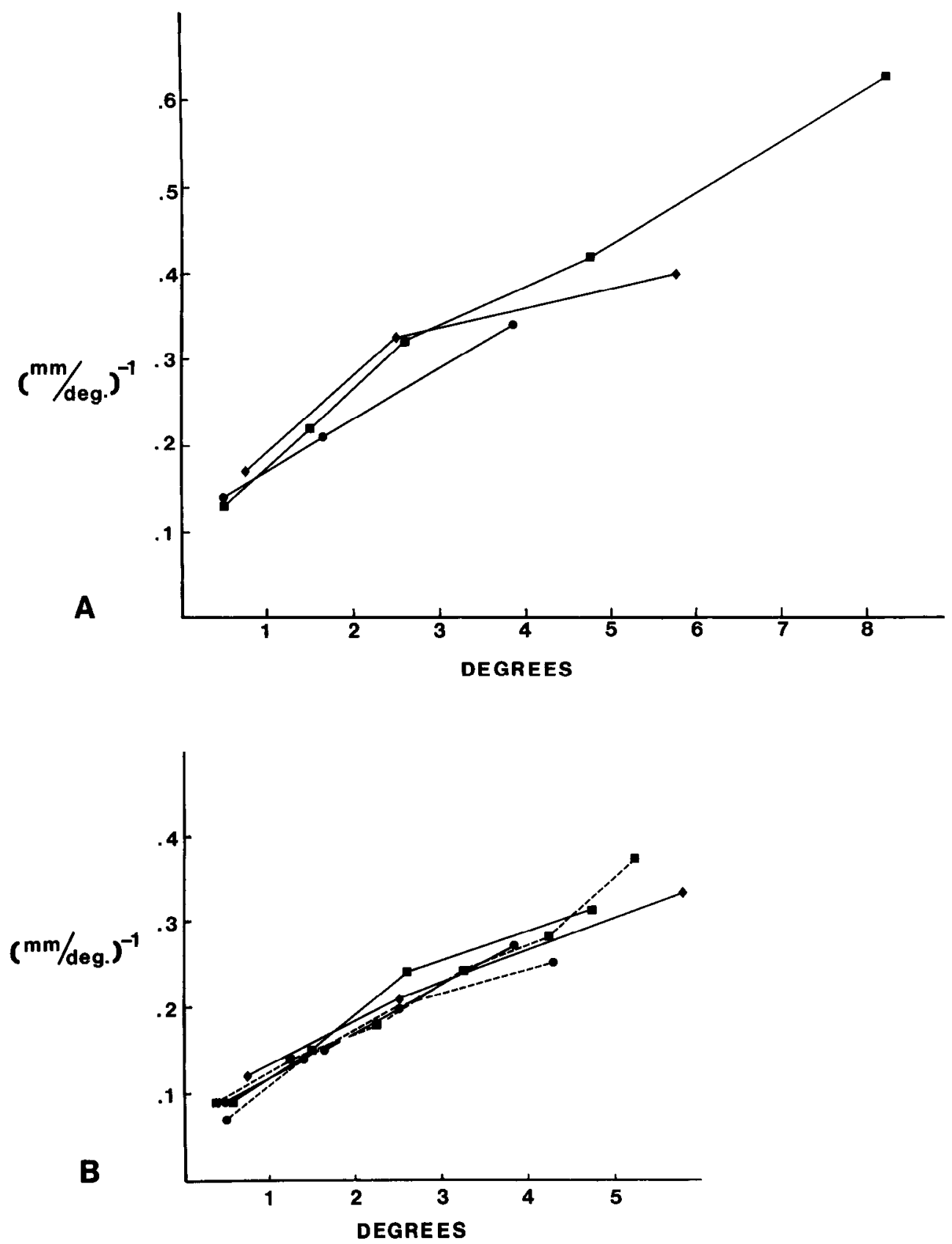

Figure 9. Magnification factor along the horizontal $(A)$ and vertical $(B)$ meridia, as computed from direct $D G$ mapping cases. For convenience, the inverse of the magnification factor has been plotted, rather than the CMF itself. The cortical magnification was computed by dividing the distance between significant loci in the DG maps by the distance between corresponding loci in the stimulus. Data from the horizontal and vertical meridia of a particular animal are indicated by use of a common symbol in $A$ and $B$. For clarity, dashed lines rather than solid lines have been used to connect some of the symbols in $B$.

fact, in that early study the vertical meridian is drawn on the lateral operculum as if it were actually expanded by a factor greater than that required to compensate for the overall difference in meridional lengths (that is, greater than about 1.25). This produces an iso-eccentric representation that is slightly bowed-in (convex relative to a foveal vantage point) at eccentricities near $5^{\circ}$, near the medial edge of the operculum. In our DG data, the presence of such a convexity of the iso-eccentric representation can be seen in virtually every operculum in which a circular retinotopic border was mapped near $5^{\circ}$. It cannot be attributed to distortion during the flat-mounting procedure. Although the bowing-in is sometimes slight, it has some theoretical significance, since such a convexity cannot be modeled by the $\log (z+a)$ conformal map (e.g., Schwart7, 1980). It is, however, consistent with the notion of an anisotropy in the CMF that is linked to the topography of ocular dominance strips (Tootell et al., 1982a; and see below).

Our best estimate of the magnification factor at the center of the fovea (about $15 \mathrm{~mm} / \mathrm{deg}$ ) is indicated by an arrow on the $\mathrm{Y}$-intercept in Figure 10. This value is essentially equal to the value one would arrive at by a linear extrapolation of the closest segment of the vertical $\mathrm{CMF}^{-1}$ (lower dotted line, Fig. 10). Extrapolation of the closest segment of the horizontal CMF would yield a different $Y$-intercept value $(9 \mathrm{~mm} / \mathrm{deg})$. Thus, even at the fovea, the cortical representation may be anisotropic.

In previous studies, the cortical magnification has typically been measured along the horizontal meridian. The slope of the $\mathrm{CMF}^{-1}$ along the horizontal meridian in our studies is very similar to those of previous studies. Results from Daniel and Whitteridge (1961), Hubel and Wiesel (1974), Guld and Bertulis 


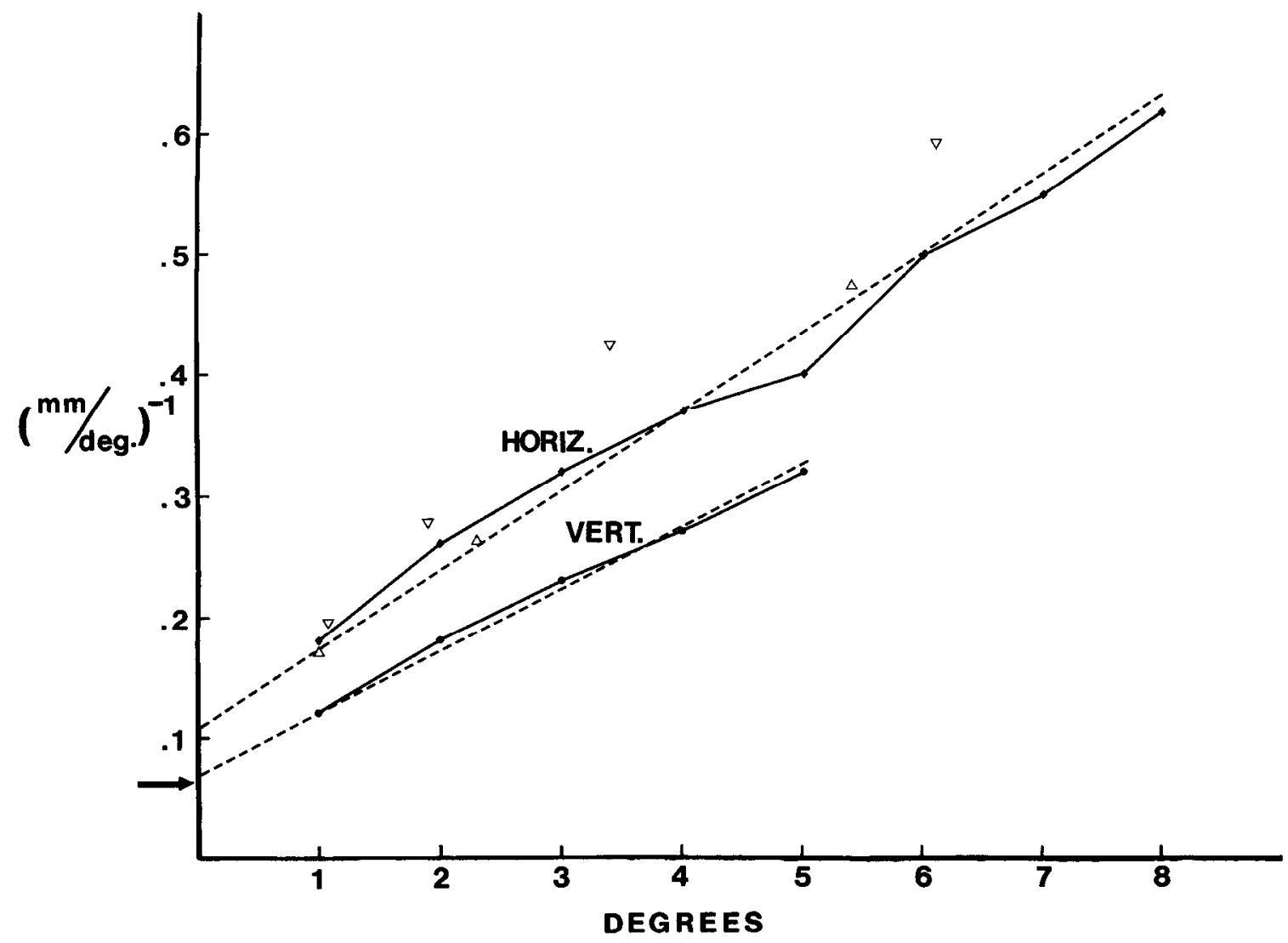

Figure 10. The solid lines indicate the average cortical magnification along either the horizontal or vertical meridia within central striate cortex. The averages are derived from the individual data graphed in Figure 9. The arrow at the lower portion of the ordinate indicates the best estimate of the foveal $\mathrm{CMF}^{-1} \mathrm{~s}$, based on other DG results from this study. At least within the central half of the striate cortex, the representation of distances in the visual field is clearly magnified along the vertical meridian relative to that along the horizontal. The dashed lines are best-fit linear regressions for the cortical magnification factors along the 2 meridia. The hollow triangles indicate data from the cortical magnification factor measured along the stimulus rings instead of the rays. Data from each of the 2 animals in which rings were analyzed are indicated by either upwardor downward-pointing hollow triangles.

(1976), and Dow et al. (1981) have been combined in Figure 11 , and those electrophysiological results are essentially indistinguishable from our DG measurements. Results from a more recent study (Van Essen et al., 1984) are also included, and the slope in this study may be slightly steeper than the average result reported previously. This minor difference may be due to the exclusive use of a smaller species of macaque ( $M$. fascicularis) in the Van Essen et al. (1984) study, as opposed to the use of larger macaque species in 3 of the other 4 electrophysiological studies, as well as in our own DG mapping experiments (see below).

There are some interesting aspects to the variation found in the CMF between different animals. Although variations between animals in Figure 9 are minimal, the one with the smallest overall CMF is also the animal with the smallest operculum. Assuming that the size of the operculum is proportional to the size of the rest of striate cortex, a straightforward compensation mechanism can be derived for the wide variation in size of striate cortex in different primate individuals (Stensaas et al., 1974; Van Essen et al., 1984). In accordance with this idea, primates with smaller brains would have CMFs scaled downwards (and $\mathrm{CMF}^{-1} \mathrm{~s}$ shifted upwards) to compensate for the relative decrease in striate area. Clearly, it is impossible to map the same number of degrees of visual angle onto different-sized striate cortices without adjusting the magnification factor in some way.
Evidence from a larger pool of data also supports this idea. In this larger sample, retinotopic data at a single eccentricity were available from monkeys that were shown a wide range of visual stimuli, all within a circular aperture of $4.5^{\circ}-9^{\circ}$ radius. This aperture was usually well-centered in the visual field, thus forming an iso-eccentric retinotopic border. Data from the horizontal meridian of 34 hemispheres (in which the stimulus was well-centered) are shown in Figure $12 \mathrm{~A}$. The data show a great deal of scatter. In some animals, a distance of $30 \mathrm{~mm}$ from the fovea represents $4^{\circ}$ eccentricity; in others, an eccentricity of $9^{\circ}$ is represented at this same cortical distance (see Fig. 12A). In Figure $12 A$, a large proportion of the variability appears to be attributable to a species difference. The cortical distance from fovea to the representation of a given eccentricity is almost always greater for the larger $M$. arctoides than for the smaller $M$. fascicularis and $M$. assamensis. However, we assume that the difference between species might simply reflect an underlying difference in average brain size between the 2 species, and so we set out to relate brain size to magnification factor in a large population from each species.

What appeared to be the most relevant index of brain size for our analyses was the distance between the foveal representation and the medial edge of the operculum along the horizontal meridian. The distance was measured in or near layer $4 \mathrm{C}$. As we had suspected, this distance was significantly larger in $M$. 


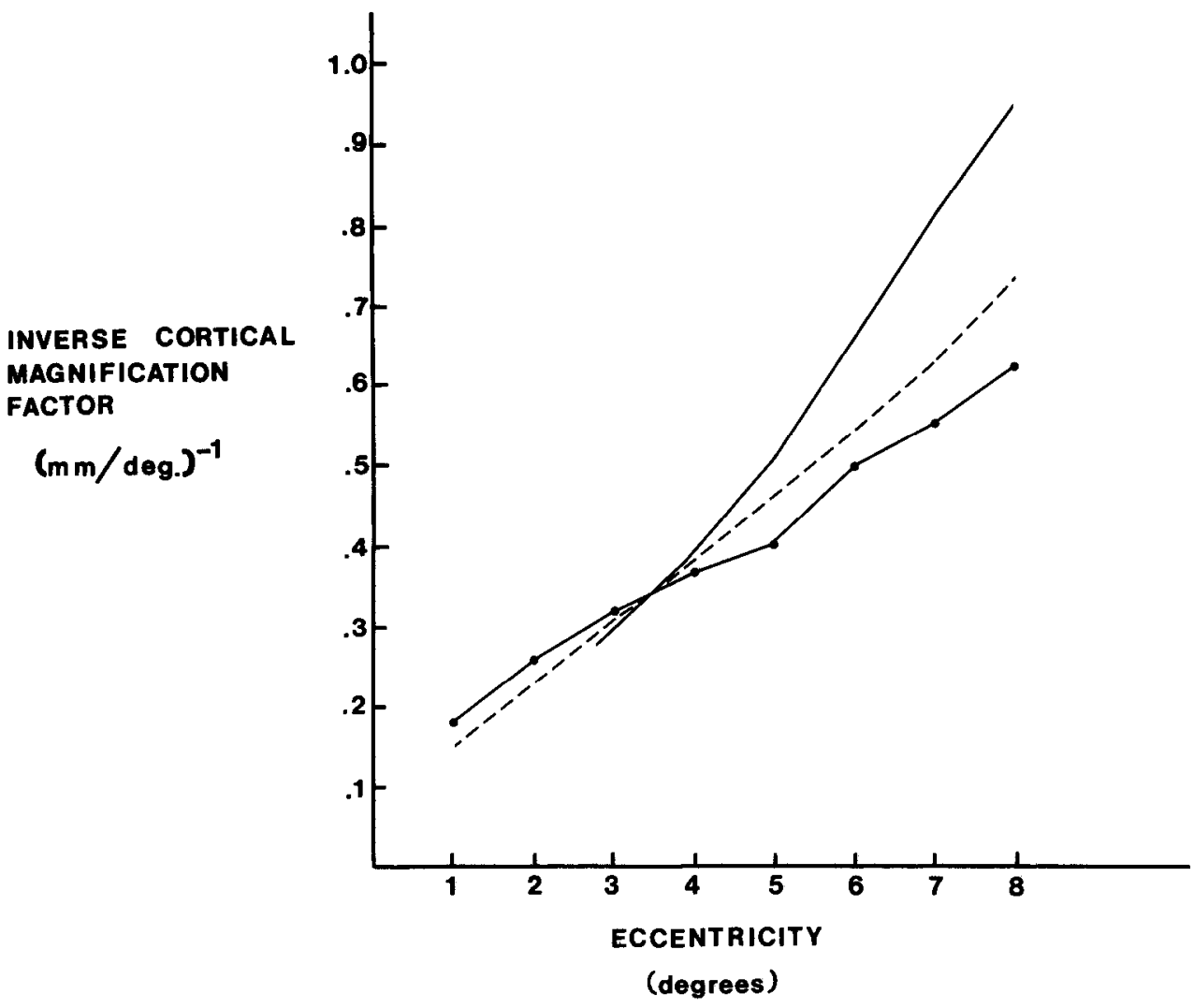

Figure 11. Comparison of magnification factors across cortex in previous electrophysiological studies with those found in the present deoxyglucose study. Data from the present study (solid line with data points) are the averages from 3 animals ( 5 hemispheres) measured along the horizontal meridian (see Fig. 9). A least-square fit to the data of Guld and Bertulis (1976), Hubel and Wiesel (1974), Daniel and Whitteridge (1961), Talbot and Marshall (1941), and Dow et al. (1981) is indicated by the dashed line (taken from Dow et al., 1981). The correspondence between our deoxyglucose data and this previous electrophysiological data is quite good. The fit is not quite as good with the data of Van Essen et al. (1984), indicated by the solid line without data points. arctoides (mean, $3.45 \mathrm{~cm}, n=24$ hemispheres, $\mathrm{SD}=0.28 \mathrm{~cm}$ ) than in $M$. fascicularis (mean, $2.91 \mathrm{~cm}, n=31, \mathrm{SD}=0.27 \mathrm{~cm}$ ) or $M$. assamensis (mean, $2.94 \mathrm{~cm}, n=14, \mathrm{SD}=0.31 \mathrm{~cm}$ ) at the $p<0.05$ level (Student's $t$ test). Because the opercular sizes of $M$. fascicularis and $M$. assamensis are indistinguishable, data from these 2 species have often been pooled in subsequent analyses.

If the CMF in different animals is in fact scaled proportionatc to brain size, then a correction for brain size should reduce the scatter in Figure $12 \mathrm{~A}$ considerably. In order to correct for brain size, we took the ratio of the cortical distance at which a particular eccentricity was mapped to the overall size of opercular striate cortex of that hemisphere, where both were measured along the horizontal meridian. (Obviously it would have been better to use the total length of striate cortex as a measure of brain size, but that information was not available.) These data are shown in Figure $12 B$. They show much less scatter than do those in Figure $12 A$, which supports the general notion that the CMF is scaled according to the size of striate cortex. Given the variation in striate cortical size, the representation of a given eccentricity may be best identified as a fixed proportion of the distance along a given meridian to the total length of that meridian, plus or minus a certain amount of additional variability due to differences in internal retinotopy.

For comparison with the population data shown in Figure 12, the detailed data from Figures 9 and 10 are replotted in the figure as dotted lines. It is evident from this comparison that the animals from which we took detailed retinotopic information may have been inadvertently sampled from the low end of the population range. Thus there may be a slightly steeper decline with eccentricity in the composite DG curve shown in Figure 11.
In the 3 animals analyzed in Figures 9 and 10, a clear difference in the CMF along the vertical and horizontal meridian is evident. In our larger population of DG cases, it also proved possible to confirm this difference in CMF between the vertical and horizontal meridians. In these cases (Fig. 10), single animals were shown stimuli containing a retinotopic border along the horizontal meridian and an iso-eccentric border at the edge of the circular stimulus. In this analysis we compared the distance from the fovea to the iso-eccentric stimulus border along the vertical meridian to that along the horizontal. These data are shown in Figure 13. Again it is apparent that the CMF along the vertical meridian is greater than that along the horizontal, at corresponding eccentricities.

Van Essen et al. (1984) have suggested that the inferior visual field is overrepresented relative to the superior field. In measurements from peripheral striate cortex, these investigators showed that the striate area devoted to the inferior visual field was, on average, about 1.25 times that devoted to the superior field. However, measurements could not be made accurately in central striate cortex because of a lack of data points and an ambiguity in the location of the horizontal meridian.

We examined central striate cortex for a similar bias in representation. In 8 hemispheres, the representation of the horizontal meridian was visible in the $D G$ pattern, there was no sulcus in the middle of the operculum, and the V1-V2 border was visible out to the medial edge of the operculum. In each hemisphere, the horizontal stimulus border was well-centered on the fovea. To compute the area of inferior and superior field representation, we took photographic enlargements from layer 4 of each of the hemispheres, cut out the superior and inferior field representations, and weighed them separately. Ratios of the inferior to the superior visual-field areas (inf/sup) had a mean 

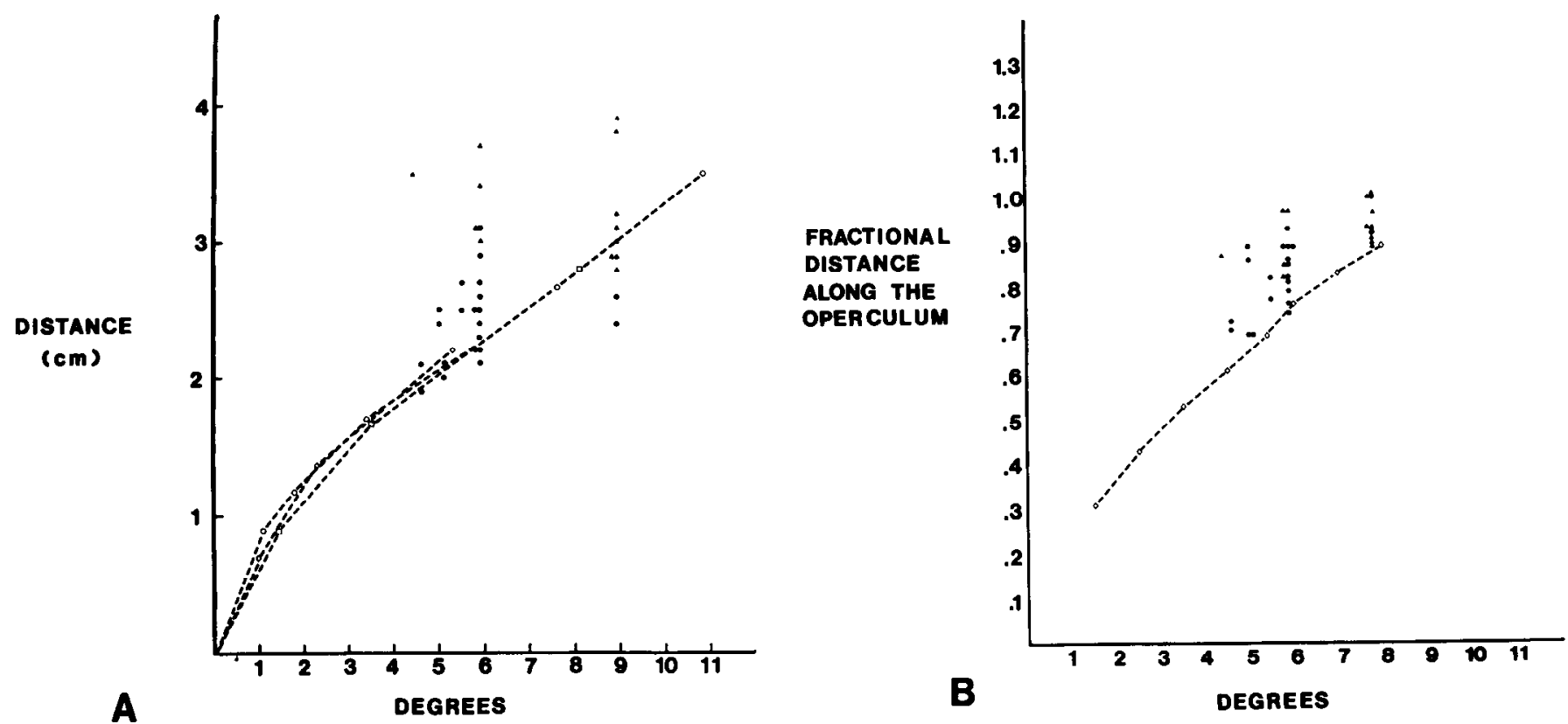

Figure 12. Cortical positions of single retinotopic loci from a large population of animals. $A$, The distance from the foveal representation to the DG border at which the outer boundary of the stimulus was represented. The half-width of each stimulus (in degrees) appears on the abscissa, and the distance (in $\mathrm{mm}$ ) along the surface of the horizontal meridian representation is plotted on the ordinate. Filled triangles, data points from $M$. arctoides, solid circles, data from $M$. fascicularis and $M$. assamensis. Wherever possible, data from the 2 hemispheres have been averaged together. For comparison, equivalent data from direct retinotopic DG cases (taken from $A$, Fig. 9) have been replotted on the same graph using unfilled symbols and dashed lines. It can be seen that there is wide variability among animals in the distance from the foveal representation at which a given retinotopic locus is represented. In general, a given peripheral locus is represented further from the fovea in $M$. arctoides than in monkeys with smaller brains (c.g., $M$. fascicularis and $M$. assamensis). $B$, Data from $A$ replotted after compensation for overall size of striate cortex. In this analysis, we assume that the length of the operculum was proportionate to the length of the entire striate cortex (which was harder to measure) along the horizontal meridian. Data points from $A$ were divided by the total distance across the operculum in each case and replotted as a ratio in $B$. Such a compensation reduces the retinotopic variability between animals, which suggests that some of the retinotopic variability (in $A$ ) is due to overall differences in size of striate cortex between animals. $B$, Averaged data from the direct retinotopic cases is again replotted, using unflled symbols and dashed lines. In both $A$ and $B$, the data from the direct retinotopic cases appear to have been sampled from the lower end of the range of interanimal variability.

of 1.22 , with a standard deviation of 0.09 . Although there is undoubtedly some error in these measurements, it is clear that the inferior visual field is relatively overrepresented in the central half of striate cortex, as it appears to be in the peripheral half.

\section{Retinotopic organization and ocular dominance strips}

Earlier we referred to the duplicate representation of the visual field in striate cortex due to the separate mapping of the 2 retinae in ocular dominance strips. Under normal binocular viewing conditions, a given single region in the visual field will be mapped onto at least 2 regions in striate cortex, one in each set of adjacent ocular dominance strips.

In one of our DG cases, there is a very graphic example of this duplicated representation of the visual field (case 9). We showed this monkey a retinotopic stimulus identical to that in Figure 1, except that in this instance both eyes viewed the stimulus. In this case, the eyes were slightly cyclorotated relative to each other. Thus, in a portion of the resultant autoradiographs one can see the DG pattern produced by both converged and unconverged viewing of a single stimulus segment in 2 layers (see Fig. 14). On the right side of Figure $14, A$ and $B$, the visual images from both eyes are convcrged almost exactly and the DG representation of the stimulus forms a single, continuous line. Stimulus disparity increases towards the left side. The single, continuous DG stripe (towards the right, Fig. 14) starts to break apart into a zipperlike pattern in the center, and finally diverges completely into 2 separate monocular representations of the single stimulus segment in the left-hand portion of Figure 14 . Where the 2 segments are fully diverged, each segment is indistinguishable from the type of $\mathrm{DG}$ pattern produced by monocular viewing conditions (see Figs. 2, 4), at least in layer $4 \mathrm{Cb}$. Since the DG segment(s) in question was mapped very close to an iso-eccentric representation, the convergence and divergence evident in the autoradiographs cannot be attributed to a constant difference in binocular disparity coupled with a difference in cortical magnification at different eccentricities.

As with other cases that were presented with this stimulus, the animal showed DG patterns in layers 3 and $4 \mathrm{Cb}$ that were quite similar. However, there is, in fact, increased uptake $b e$ tween the 2 slightly diverged segments in layer 3 (see Fig. 14A), which does not appear in the input to layer $4 \mathrm{C}$ (Fig. 14B). The increased uptake between the upper-layer segments is presumably a special case of binocular "border enhancement," described in the preceding paper (Tootell et al., 1988a). The concentration of heightened uptake between (rather than outside of) the disparate ring representations raises the possibility that the border enhancement is related to the perceptual fusion of double (monocular) images into a single binocular percept. Obviously more information is needed to verify this idea. In the present context, the important point is that the increased uptake between the upper-layer ring segments is most probably due to 


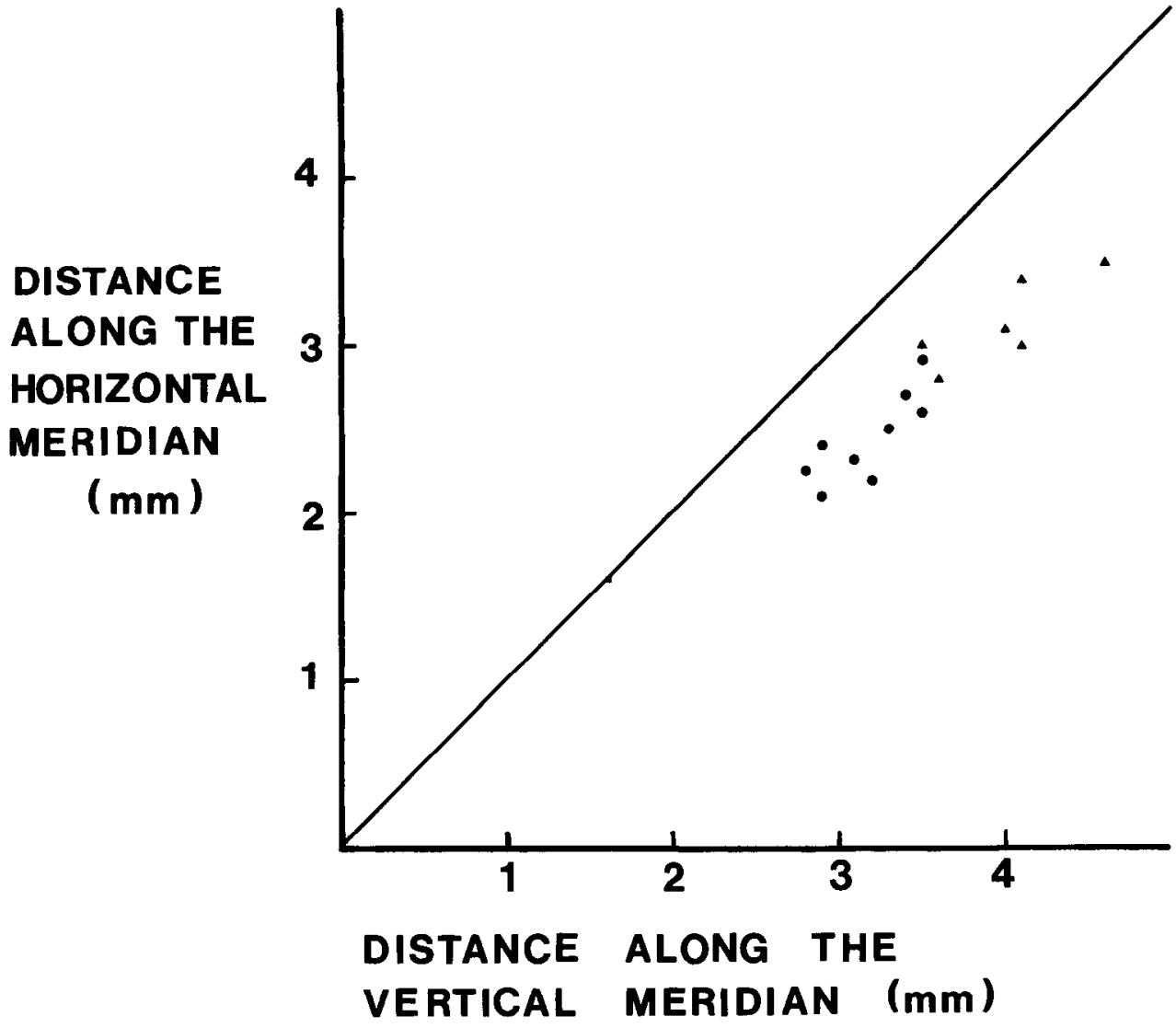

Figure 13. Differences in the magnification factor along the horizontal and vertical meridian for a larger pool of monkeys. In a number of monkeys, the DG boundary of a single iso-eccentric point was available across both the horizontal and at least one vertical meridian, and these distances are plotted. As in the direct DG mapping cases, the representation of a single eccentricity was mapped further from the fovea along the vertical meridian than along the horizontal meridian. The solid line running at $45^{\circ}$ to the $\mathrm{X}$ and $\mathrm{Y}$ axes represents a horizontal-vertical ratio of 1 . a nonvertical transmission of retinotopic information in striate cortex between layer $4 \mathrm{Cb}$ and layers $2+3$.

\section{Local magnification and ocular dominance strips}

The duplicated representation of the visual field in each ocular dominance realm places certain constraints on cortical mapping. Without any adjustment of the magnification factors relative to the ocular dominance strips, the 2 maps of the visual field from each eye will cause the overall cortical map to be expanded along axes that are locally perpendicular to the ocular dominance strips. One simple way of avoiding this eventuality is to suppose that the magnification factor is halved perpendicular to the long axis of the ocular dominance strips (Hubel et al., 1974; Hubel and Wiesel, 1977). We refer to the proposed halving of the magnification factor (perpendicular to the ocular dominance strips) as the " $2: 1$ model," and to the model that proposes no local decrease in magnification as the " $1: 1$ model."

This idea has an additional wrinkle. Two monocular maps arrive for layer $4 \mathrm{C}$. Because many cells in the extragranular layers are binocular, one might presume that these 2 monocular maps arc intcgrated into a single binocular map in the cxtragranular layers. According to this idea, a 2:1 representation in layer $4 \mathrm{C}$ would become a $1: 1$ representation in the extragranular layers, and vice versa. However, for the DG data, the ocular dominance and the retinotopic topographies in layer $4 \mathrm{C}$ are similar to those in other layers, except for differences in resolution. In particular, there is no obvious integration of the 2 monocular maps into a single binocular map in the extragranular layers. Thus, all our DG analysis is carried out on one or both of the monocular input maps. Of course, measurements of the binocular retinotopic organization can be made by simply summing the DG data from both sets of ocular dominance strips (see below).

Since the ocular dominance strips often tend to run in long, parallel bands, the 1:1 model would suggest that the overall shape of striate cortex would be expanded in a direction perpendicular to the average long axis of ocular dominance strips. Reasoning that ocular dominance strips run in characteristic directions across cortex, an attempt has been made to discriminate between the 1:1 and 2:1 models by analzying the shape of the lateral operculum (Sakitt, 1982). Other neural models have also involved tacit assumptions about "the" shape of the striate cortex (e.g., Schwartz, 1980).

However, we have found that the shape of the lateral operculum varies widely between animals (see Fig. 15). Figure 15 shows the outline of the V1-V2 border along the lateral operculum in 2 different animals (cases 36,41 ). Both operculae are those of adult macaques and are shown at the same scale. The angle formed by the superior versus inferior vertical meridian borders was approximated by overlaying a line parallel to the V1-V2 border: these angular estimates are about $65^{\circ}$ and $35^{\circ}$ in Figure 15, $A$ and $B$, respectively. In the face of such wide variability between animals, it is hard to justify any conclusions based on "the" shape of the macaque operculum.

In several electrophysiological mapping studies, shifts in receptive-field position have been described at the borders of ocular dominance columns in layer 4C (Hubel et al., 1974; Hubel and Wiesel, 1977; Blasdel and Fitzpatrick, 1984). Such shifts would be a natural consequence of a complete mapping of the visual field: points that are adjacent in one retina will presumably be separated by several hundred microns in striate cortex when interrupted by an ocular dominance strip from the 

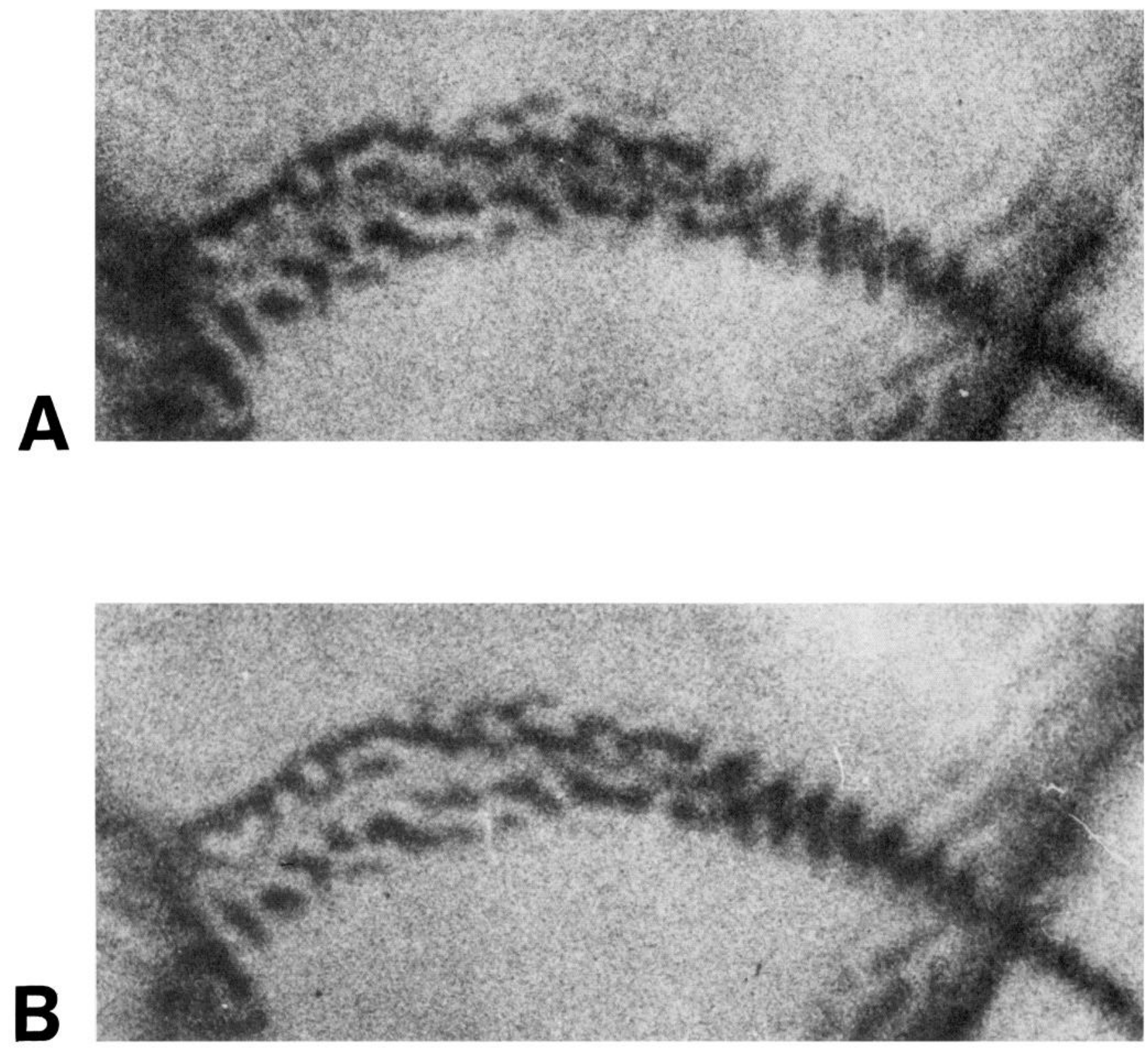

Figure 14. Binocular representation of a single retinotopic segment. $A, B$, DG autoradiographs produced by a stimulus segment of blinking black and white checks on a gray background (e.g., Fig. 1), taken from sections cut parallel with the surface of flattened striate cortex at topographically corresponding regions. $A$, Taken from layer $3 ; B$ cuts through layer $4 \mathrm{Cb}$. On the right-hand sides, the eyes are converged, so that the representation of the stimulus segment forms a single solid line in cortex. Towards the left, the eyes become progressively misaligned, so that the retinotopic representation of the stimulus segment becomes progressively isolated in its separate ocular dominance arrays. It can be seen that in layer $3(A)$, a pattern of diffuse, elevated uptake is found between the binocularly diverged retinotopic segments at the left. Such a diffuse pattern of elevated DG uptake does not occur between diverged segments in layer $4 \mathrm{Cb}(B)$ nor in other striate layers, such as $4 \mathrm{~A}, 4 \mathrm{~B}, 4 \mathrm{Ca}, 5$, or 6 . The spread of elevated uptake is much greater than would be expected entirely on the basis of the slight decrease in monocular resolution of layer 3 relative to $4 \mathrm{Cb}$ (see Fig. 18). This appears to be a special case of the DG border enhancement described in Tootell et al. (1988a). Scale bar, $2.5 \mathrm{~mm}$.

other eye. However, there is a certain amount of receptive-field scatter in electrophysiological mapping studies such as this, and this scatter obscures the evidence for (or against) such receptivefield jumps at the borders of the ocular dominance strips.

An analog of these electrophysiological shifts in receptivefield position can also be seen in the DG results. Since the DG reflects the activity of many neurons at a time, such receptive- field scatter is "averaged" out; thus, the shifts in receptive-field position are in some ways easier to demonstrate with the DG technique. From the anatomical results, we can also say something about the presence and size of the ocular dominance anisotropy. The DG results also allow us to definitively rule out the possibility of an incomplete (half-) mapping of the visual field (i.e., regions of the visual field are represented in only one 
or another ocular dominance strip). Though such a proposal seems teleologically absurd, it is one possible way to preserve overall shape constraints in striate cortex without incorporating a 2:1 anisotropy in the CMF.

In the case illustrated in Figure 4 (case 18), the fifth stimulus ring does not appear on the operculum because it was mapped on the underside of the operculum (see Fig. 16). Here, the stimulated portion of the ocular dominance strips runs almost parallel to the iso-eccentric representation of the stimulus ring (Fig. $16 \mathrm{C}$ ). In such an instance, where the 2 are almost parallel, a model of complete visual-field duplication would predict that at the point where the stimulus representation meets the border of an ocular dominance strip, DG activation should immediately jump over to the next available strip from the same eye (see Fig. 16B). A model of incomplete (half-mapped) visual-field representation would predict no such skipping (see Fig. 16A). The DG result in $14 \mathrm{C}$ is entirely in accord with the model of complete visual-field representation. The "skipping" of DG activity across the unstimulated eye dominance strip is the anatomical correlate of shifts in receptive-field position at the borders of ocular dominance strips in electrophysiological mapping studies (Hubel et al., 1974; Hubel and Wiesel, 1977; Blasdel and Fitzpatrick, 1984).

At ring-and-ray intersections such as this, where nearby DG segments run either parallel or orthogonal to the ocular dominance strips, we can also estimate the local magnification factor along corresponding axes of the strips. If there is no decrease in the magnification factor perpendicular to the strips (the $1: 1 \mathrm{mod}-$ $\mathrm{cl})$, then the width of DG scgments that run almost parallcl with the strips should be twice the width of intersecting segments running perpendicular to the strips. On the other hand, if the CMF is halved perpendicular to the strips (the 2:1 model), the width of all DG segments should be equal, irrespective of the local ocular dominance topography.

In order to resolve this issue, we need only measure the width of DG segments that run near-parallel or near-perpendicular to the ocular dominance strips, at the same or nearby eccentricities. Width measurements are straightforward where ocular dominance strips cross the rays or rings perpendicularly; in this case, measurements can be made entirely within a single ocular dominance strip, along the long axis of the strip. Measuring the width of DG segments that run parallel with the ocular dominance strips is more complicated, since this measurement may include more (or less) than one ocular dominance strip. In these cases, the width of the regions of high DG must be doubled to compensate for the fact that our monocular stimulation conditions activated only half of the duplicated visual-field representation (see below).

This is illustrated in Figure 17, which shows a number of examples of DG rays and rings produced by monocular stimulation. In Figure $17, A-F$, all rings and rays are equated in terms of stimulus size and eccentricity. In Figure 17, $A$ and $B$ show intersecting rays and rings in which the rings run nearparallel to the ocular dominance strips and the rays run nearly perpendicular. (Thus, the true "binocular width" of retinotopic rings in Fig. $17, A, B$, is actually equal to the width of the stimulated strips plus an equally wide area of unstimulated uptake between the strips, assuming no "half-map.") In both cases, it can be seen that the width of the DG ring representation is greater than that of the ray representation. This does not indicate that the radial magnification is different from that of the isoeccentric representation, however. Figure $17 C$ shows a segment

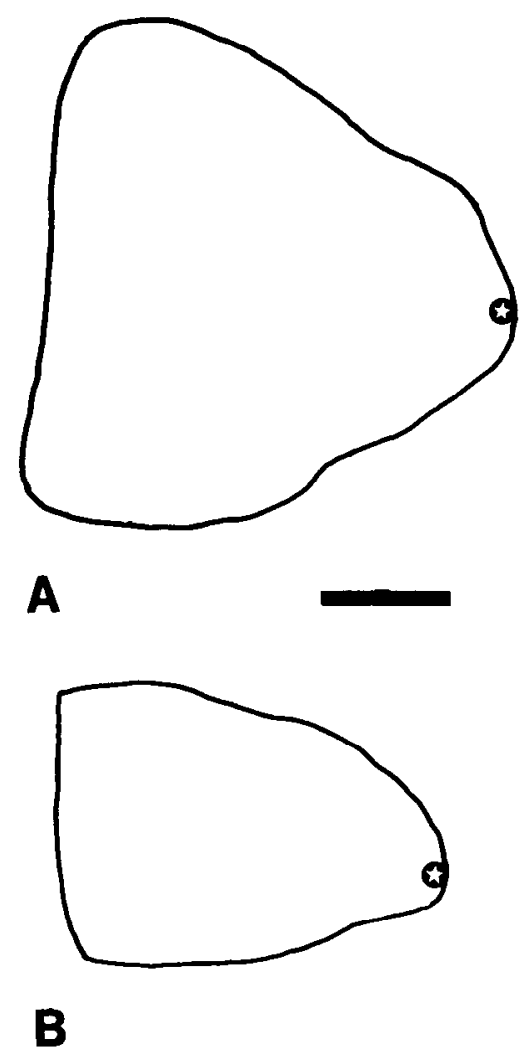

Figure 15. Differences in the overall shape of opercular striate cortex. The diagrams in $A$ and $B$ have been drawn from flattened tangential sections cutting through layer 4 in 2 different animals. The fovea is represented toward the right (star), the medial edge of the operculum is represented towards the left, and the V1-V2 border forms the outer bound on either side of the foveal representation. Both sections have been drawn to the same scale. The major difference in opercular shape ( $A$ and $B$ ) cannot be attributed to differential distortions during flattening (see text). The real biological variability in opercular shape makes estimates of anisotropy based on overall opercular shape hazardous, if not silly. Scale bar, $1 \mathrm{~cm}$.

of the same ring shown in $17 \mathrm{~B}$, but in $17 \mathrm{C}$ the ring intersects the ocular dominance strips orthogonally, so the DG representation of the ring becomes thinner. Measuring across all ring and ray representations of the type illustrated in Figure 17, $A$ $C$, we find that the width of DG segments running perpendicular to the ocular dominance strips is about equal to the combined width of the stimulated (dark) segments running parallel to the ocular dominance strips. Since the visual field is duplicated, the elongation of the ocular dominance strips means that the overall cortical representation is approximately doubled perpendicular to the ocular dominance strips, in rough accord with the $1: 1$ model.

Other evidence is also incompatible with a halving of the magnification factor perpendicular to the ocular dominance strips (the 2:1 model). Figure 17, D-F, shows 3 radii taken from the same hemisphere and eccentricities. The radial segment that crosses ocular dominance strips at about $90^{\circ}$ is the longest and thinnest. The other 2 radial DG segments run more parallel (or randomly) relative to the ocular dominance strips, so they are shorter and wider.

All this evidence indicates that there is some expansion of the overall cortical representation perpendicular to the ocular dominance strips relative to that parallel to the strips. Thus the 

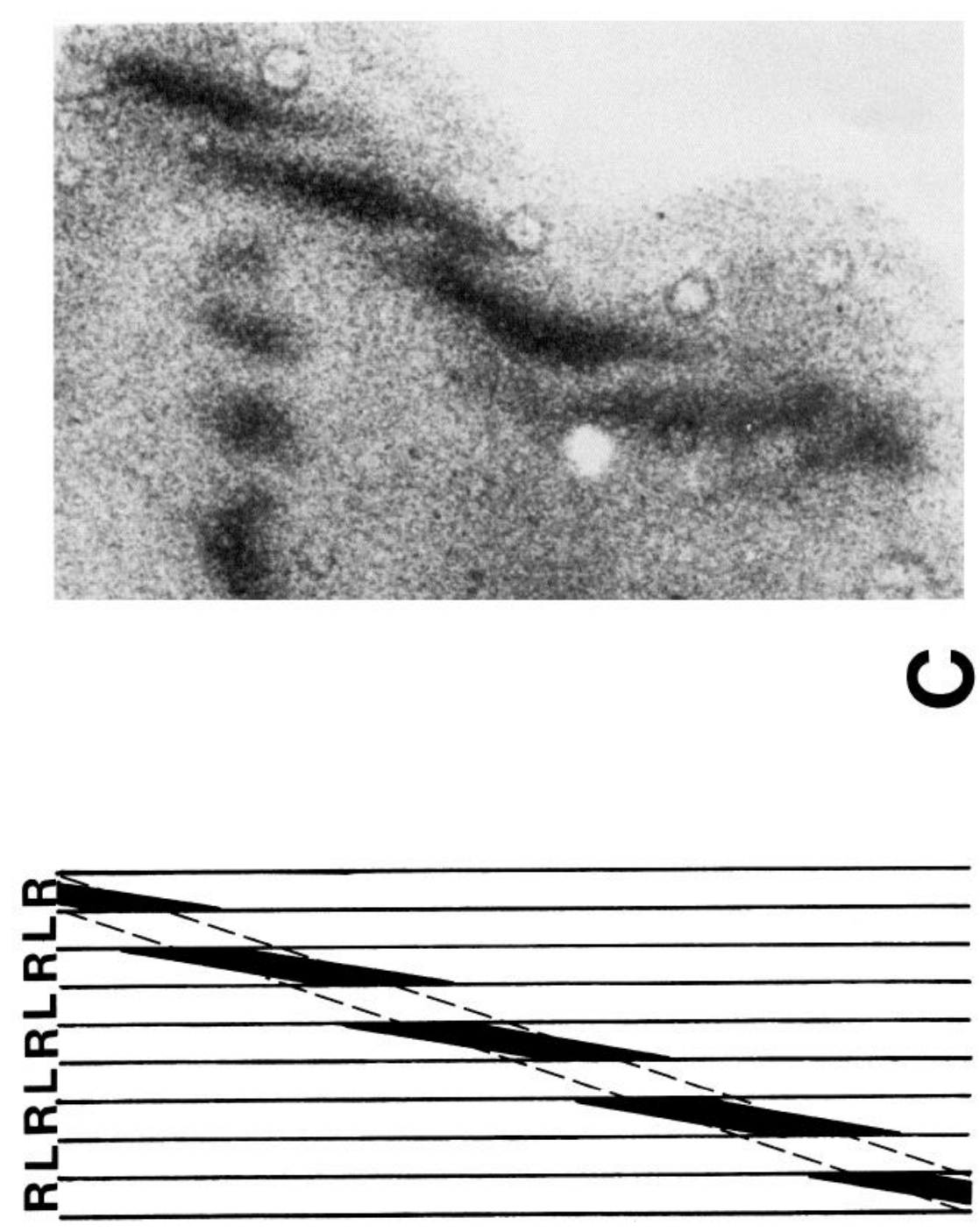

$\boldsymbol{m}$

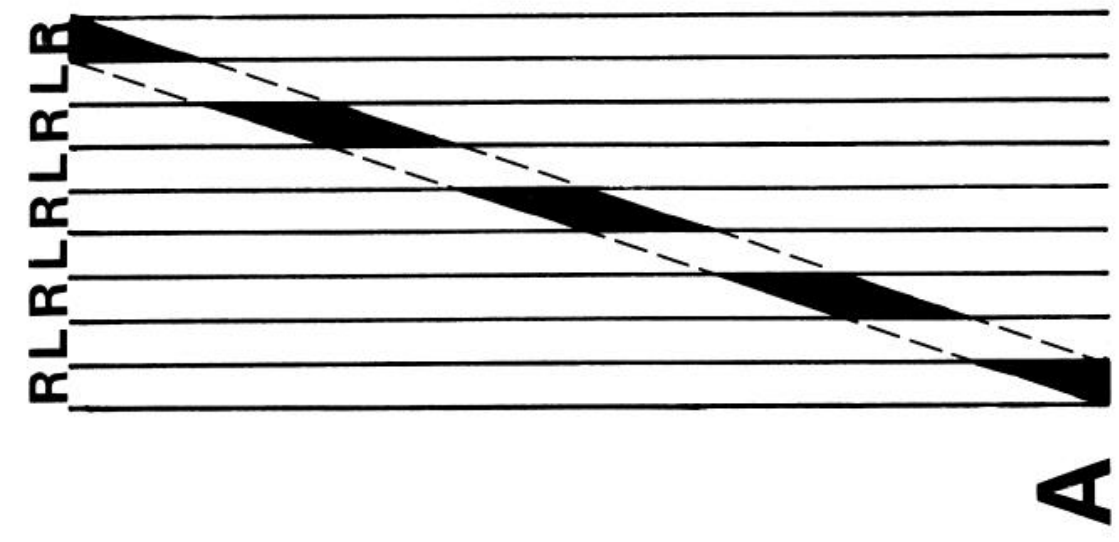

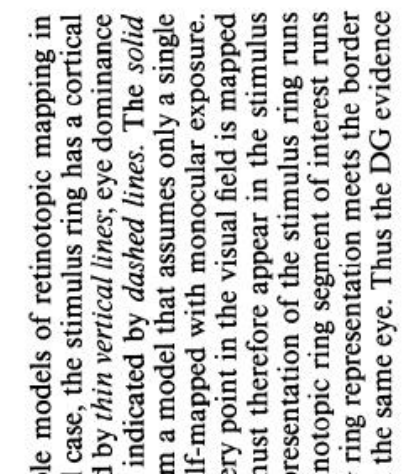

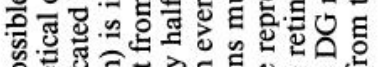

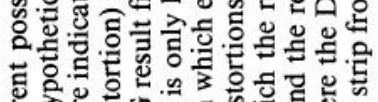

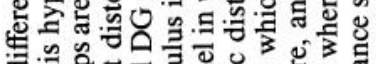

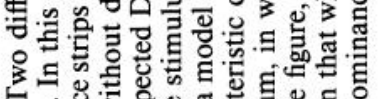
$\infty$. > Sิ원.

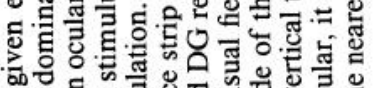

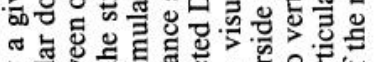

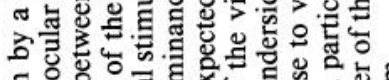

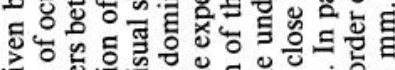
它牙.

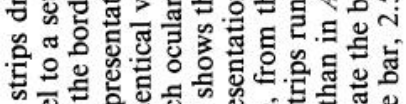
कि

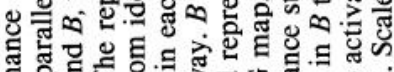

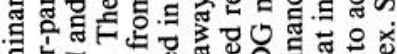

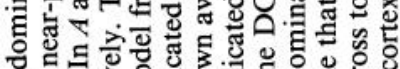

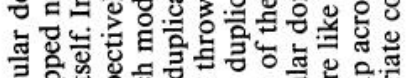

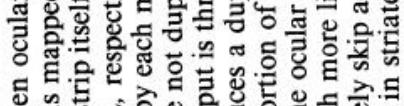

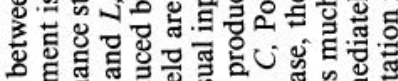

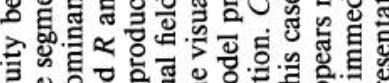

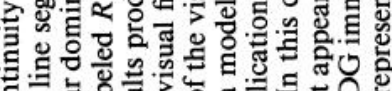

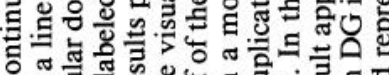

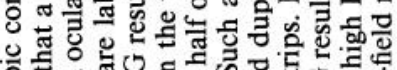

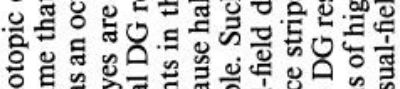

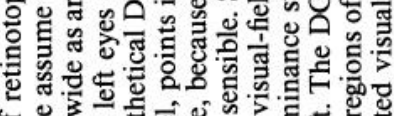
๘

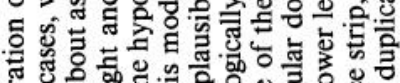

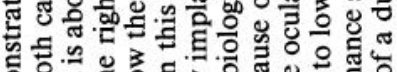

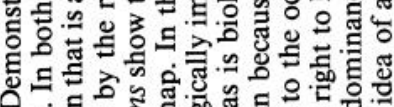
国

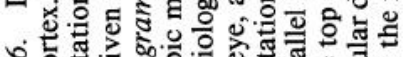

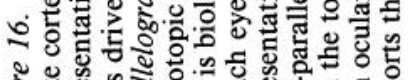

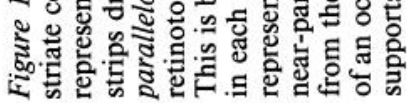



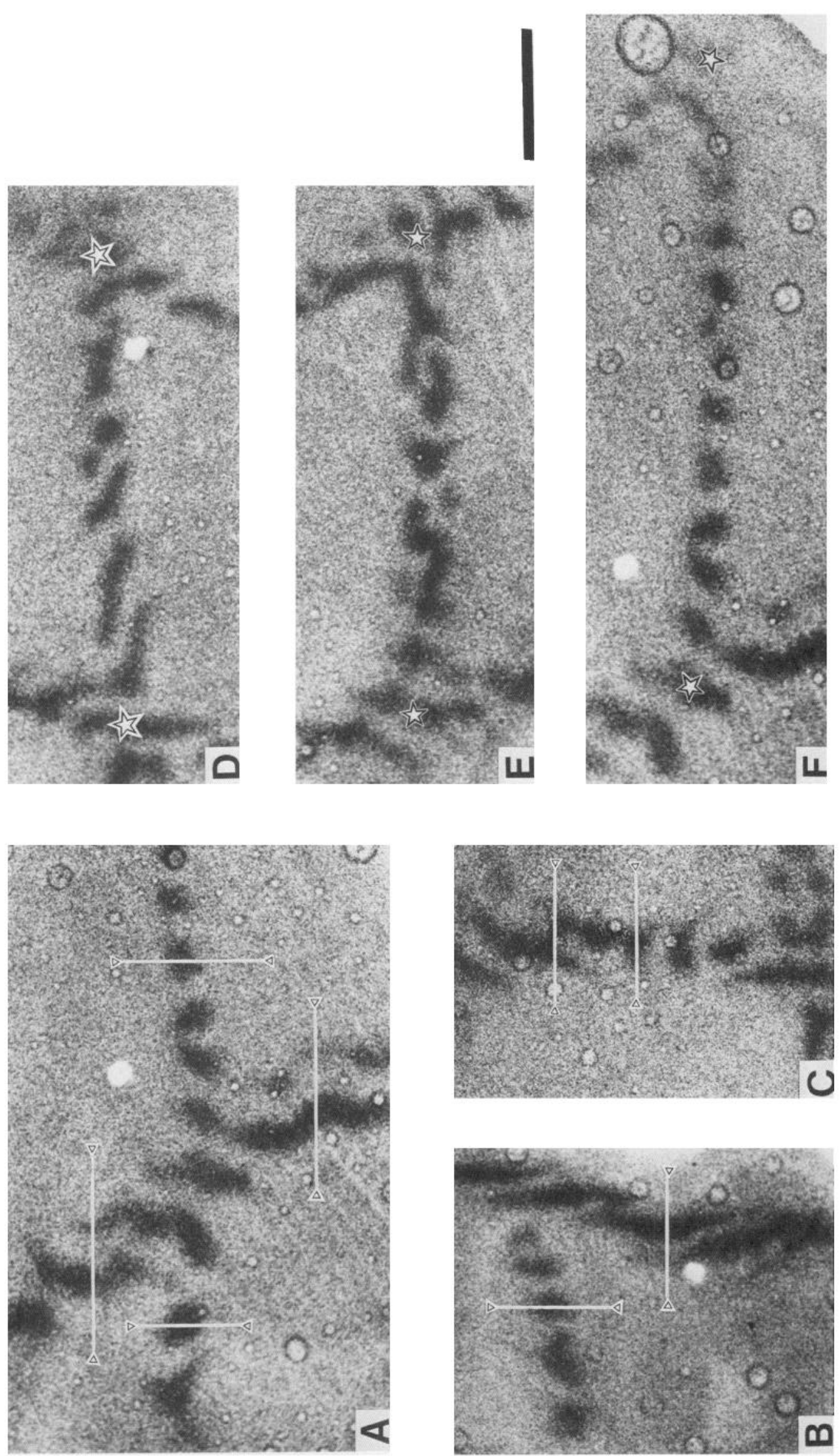

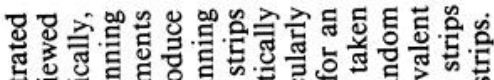
t. 웡

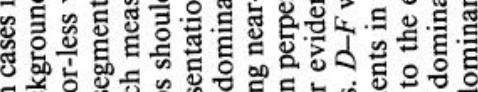

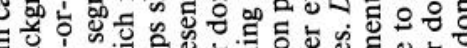

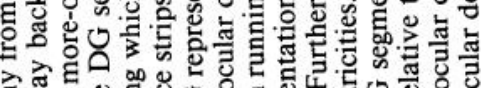

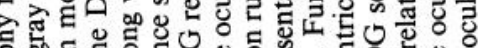

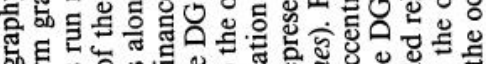

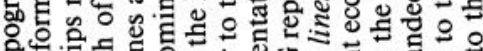

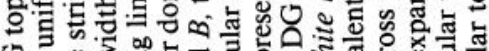
ช 83 .

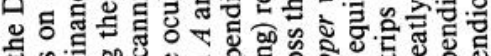

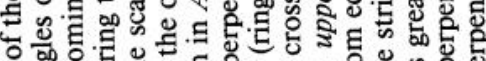

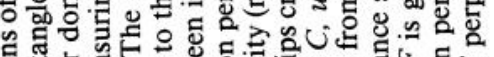
等

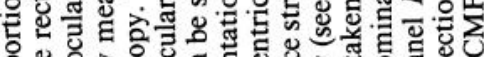

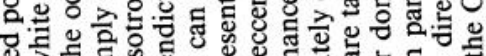

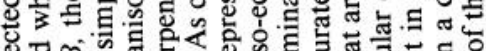

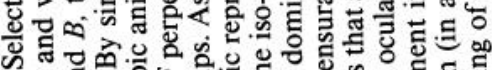

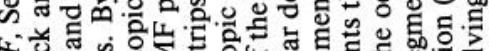

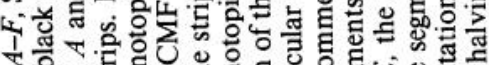
Tे

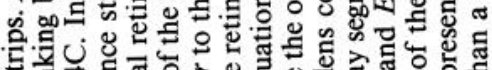

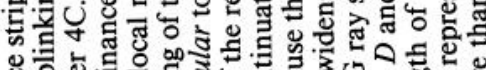
8.

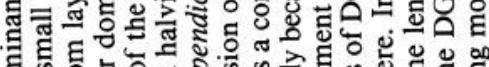

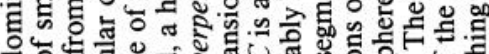

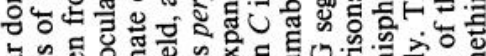
t⿹

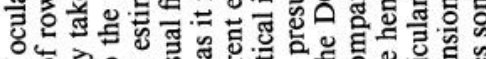

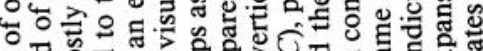

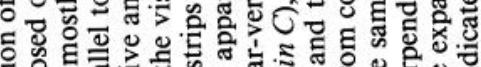

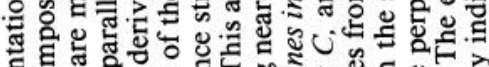
든

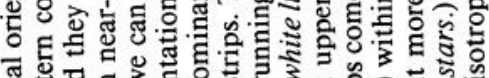

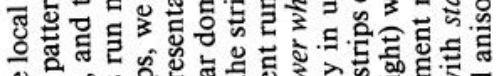
웅

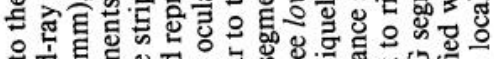

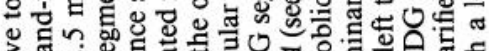
象 密. .

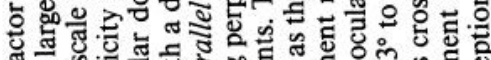
象

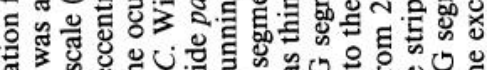
势

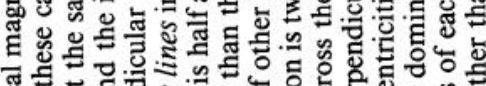
于. 8 은 可 은

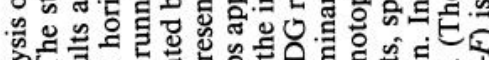

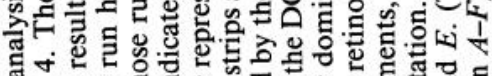

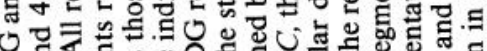

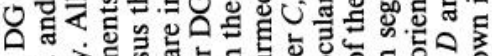

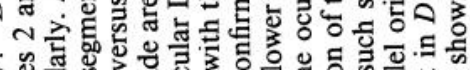

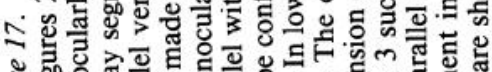

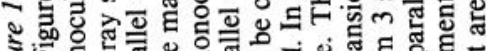

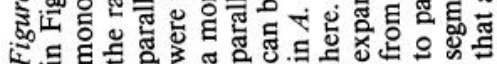


2:1 model is inappropriate. Because the deoxyglucose techniques we have used in this study have a resolution ambiguity of about $100 \mu \mathrm{m}$ (half-amplitude, full-width), it is possible that the expansion perpendicular to the ocular dominance strips does not amount to a full doubling. This would allow a slight decrease in the magnification factor perpendicular to the ocular dominance strips (the ca. 1.5:1 model; see Tootell et al., 1982a; Horton, 1984). However, the 1:1 model is the most parsimonious model, and within the measurement limits our evidence is in accord with this model.

\section{Physiological spread}

In this section we report on experiments on the physiological spread of activity, as measured with DG. Since single-unit and DG techniques may reflect activity originating in different cellular compartments, it is possible that the 2 techniques will yield different figures for the extent of physiological spread within a given layer. Where data from both the DG and electrophysiology are available, they are quantitatively compared in Retinotopic spread and acuity limits, below. In general the correspondence is good.

Measurements of the horizontal spread of functional activity have been expressed either as the minimum distance between the centers of nonoverlapping receptive fields (Hubel and Wiesel, 1974, 1977; Blasdel and Fitzpatrick, 1984) or as the cortical point image (that is, the amount of cortex stimulated by a point of light on the retina) (Dow et al., 1981; Van Essen et al., 1984). The 2 measures are conceptually similar, except that the cortical point image is expressed in square millimeters and the nonoverlap distance in millimeters. Both measures vary as a function of the average size and scatter of receptive fields in a given layer. However, a single point of light in the retina may or may not summate linearly within different striate receptive fields (cf. complex vs special complcx cclls; Palmer and Rosenquist, 1974; Gilbert, 1977). These differences in the linearity of summation may not be reflected as acutely in measurements of the minimum non-overlap distance as they would be in measurements of the cortical point image, so the 2 measures of horizontal spread may not be experimentally equivalent. To be safe, we measured DG analogs of both concepts.

\section{Cortical point image}

We first describe our DG analog of the cortical point image measurements. The DG measurements were made by displaying blinking black and white checks on a uniform, unvarying gray background. For convenience, measurements of DG borders in these cortical point image experiments are expressed in terms of length $(\mathrm{mm})$ rather than area $\left(\mathrm{mm}^{2}\right)$. In all of these experiments, the stimulation was monocular.

In the most straightforward test of the cortical point image, small, square checks $\left(0.11^{\circ}\right.$ on a side) were arranged $1.2^{\circ}$ apart along the horizontal meridian and along 2 oblique rays, at eccentricities greater than $1^{\circ}$ (case 26). For reasons of economy, this pattern was combined with one from another experiment. Both the strip and the small fickering checks were presented on a stable gray background of the same mean luminance (see Fig. $8 A$ ). The large vertical strip is irrelevant to the present discussion.

The DG pattern produced by the blinking checks can be seen faintly in Figure $8 B$, especially along the parafoveal horizontal meridian. The DG check representations are most obvious in layer 4 , and they fade out progressively in the upper and lower layers. Because the checks are of constant stimulus size, the cortical representation of each check is larger as it gets nearer the fovea. The larger check representations near the fovea produce a higher contrast in the DG pattern, and a greater extension of the 2DG pattern into the reaches of the upper and lower layers. The variation in check representation with eccentricity may also be exaggerated by variations in the cortical point image (see below).

The fadeout towards the peripheral representation, and the decreased uptake in the upper and lower layers, is what one would expect if there were an inverse relationship between DG uptake and average receptive-field size. In this model, the decrease in DG uptake with increasing eccentricity is due to the steady increase in average receptive-field size away from the fovea. The same model would predict that an hourglass-shaped distribution of receptive-field size across laminae would produce the highest DG uptake in layer 4 , at the narrow portion of the hourglass. However, another very similar experiment yielded a better view of the situation, and some of the laminar differences in DG point image results are more complicated than this.

In the experiment illustrated in Figures $1-3$, an animal was shown a pattern of slightly larger rectangular checks, set adjacent to each other along one side (case 13). In all other respects, the checks in Figure 1 were quite similar to those used in the more straightforward point image mapping experiment (illustrated in Fig. 8). However, the cortex of the case shown in Figures 1-3 (and the interblob distances within that cortex) was larger than that illustrated in Figure 8. Thus it is possible to resolve the DG results in Figures 1-3 better than in Figure 7.

Laminar differences in the DG representation of an iso-eccentric $\left(5.4^{\circ}\right)$ ring of checks are shown in Figure 18. The DG segment fades out completely in upper layer 2 and in layer 6 , as does the 2DG pattern produced by small, single checks (case 26). A numbcr of fincr details can also be resolved in the present case. The DG pattern is highest in contrast (and the borders most sharply defined) in layer $4 \mathrm{Cb}$. In layers $4 \mathrm{Ca}$ and $4 \mathrm{~B}$ the borders are more diffuse, although the contrast of the $D G$ pattern is still high in these layers. The relative fuzziness of the border in $4 \mathrm{Ca}$ and $4 \mathrm{~B}$ presumably reflects the increased size of the axonal arborization in $4 \mathrm{Ca}$ (relative to $4 \mathrm{Cb}$ ), as well as the strong projection to 4B from 4Ca (Lund and Boothe, 1975; Blasdel et al., 1985; Fitzpatrick et al., 1985). In layer 3, the DG representation is fairly high in contrast, and again the borders are fairly sharp. Presumably this reflects the strong projection to layer 3 from $4 \mathrm{Cb}$, and a relatively weaker projection to layer 3 from $4 \mathrm{Ca}$ and $4 \mathrm{~B}$. In layer 5 , the DG segment is low in contrast and extremely diffuse.

In order to quantify the sharpness of the borders in Figure 18 , densitometric measurements were made parallel with the long axis of the ocular dominance strips in various layers. Thus, these scans crossed retinotopic (but not ocular dominance) boundaries. Optical density values thus obtained were converted to levels of DG uptake by using autoradiographic standards. The sharpness of the DG borders was measured by fitting linear approximations to the 1-dimensional uptake scans and by expressing this falloff as a half-amplitude at half-width (see Fig. 19). Such figures can also be interpreted as the radius at half-amplitude of the cortical point image. Such an approximation to the falloff in layer $4 \mathrm{Cb}$ amounted to about $140 \mu \mathrm{m}$ across the cortex. Given the measurement resolution, the falloffs in $4 \mathrm{Ca}$ and $4 \mathrm{~B}$ were essentially indistinguishable at 350 and 330 $\mu \mathrm{m}$ of cortex, respectively. The falloff in layer 3 was about 240 

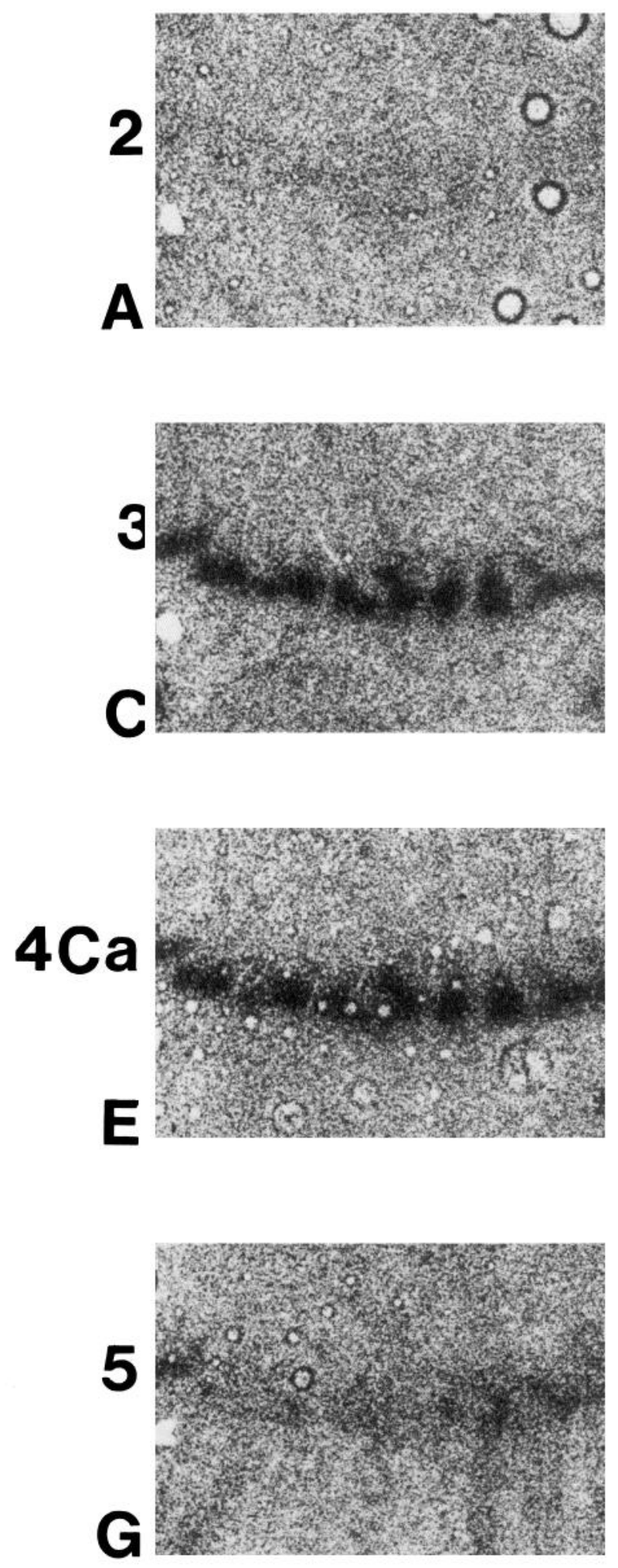
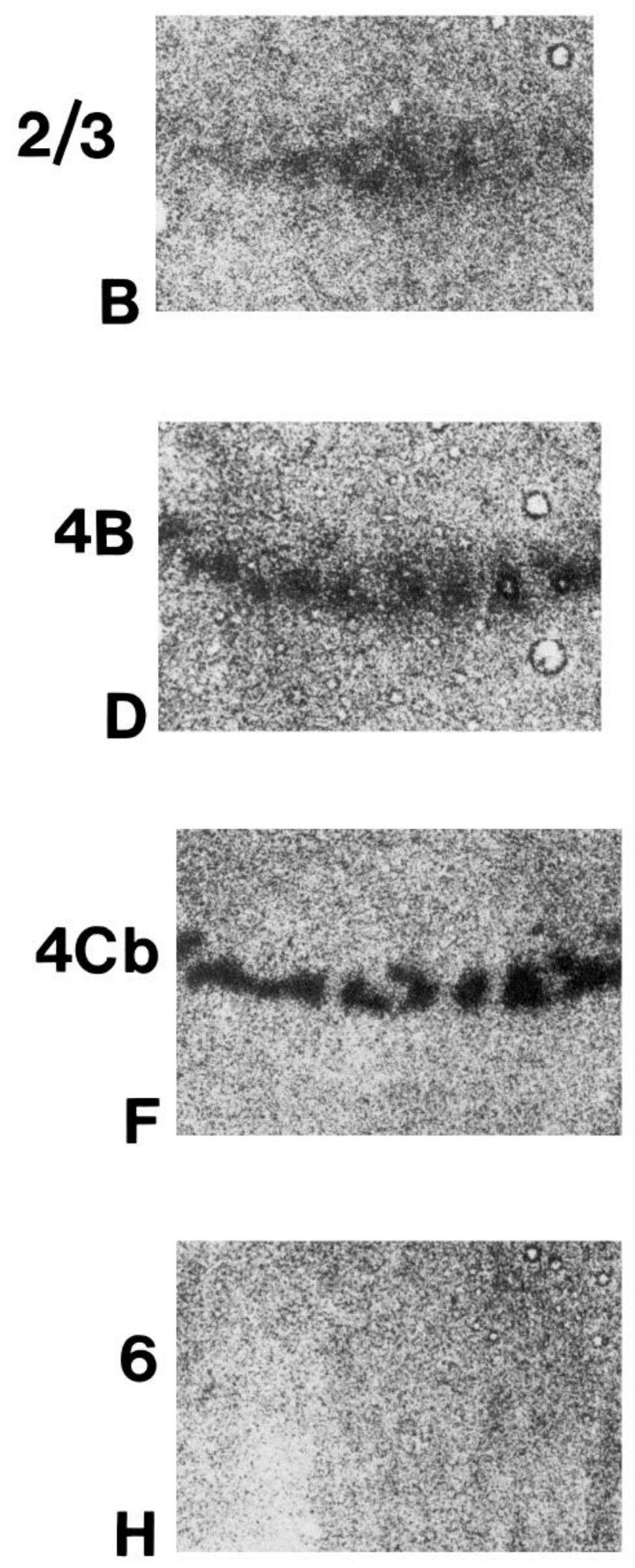

Figure 18. Laminar variations in topographically corresponding portions of the DG result from "cortical point image" cases. The stimulus in these cases was a row of small black and white dots on a uniform gray background, viewed monocularly. The borders of this DG segment are sharpest (and the contrast is highest) in the parvorecipient layer $4 \mathrm{Cb}$. In the magnorecipient layer $4 \mathrm{Ca}$, the contrast is also high, but the DG borders are much blurrier. This is in accord with the extensive spread of magnocellular afferent arborizations in $4 \mathrm{Ca}$ (relative to parvocellular afferent arborization in $4 \mathrm{Cb}$ ). Layer $4 \mathrm{~B}$ derives much of its input from $4 \mathrm{Ca}$, and the retinotopic map in $4 \mathrm{~B}$ is slightly lower in contrast and about as fuzzy as that in $4 \mathrm{Ca}$. In layer 3, the DG map is sharper than that in $4 \mathrm{Ca}$ and $4 \mathrm{~B}$, which seems to rule out a strong input from magnocellular-dominated layers. In layers above lower 3 and below layer $4 \mathrm{C}$, the contrast and the resolution of the DG segment drops off sharply. Scale bar, $5 \mathrm{~mm}$. 
A
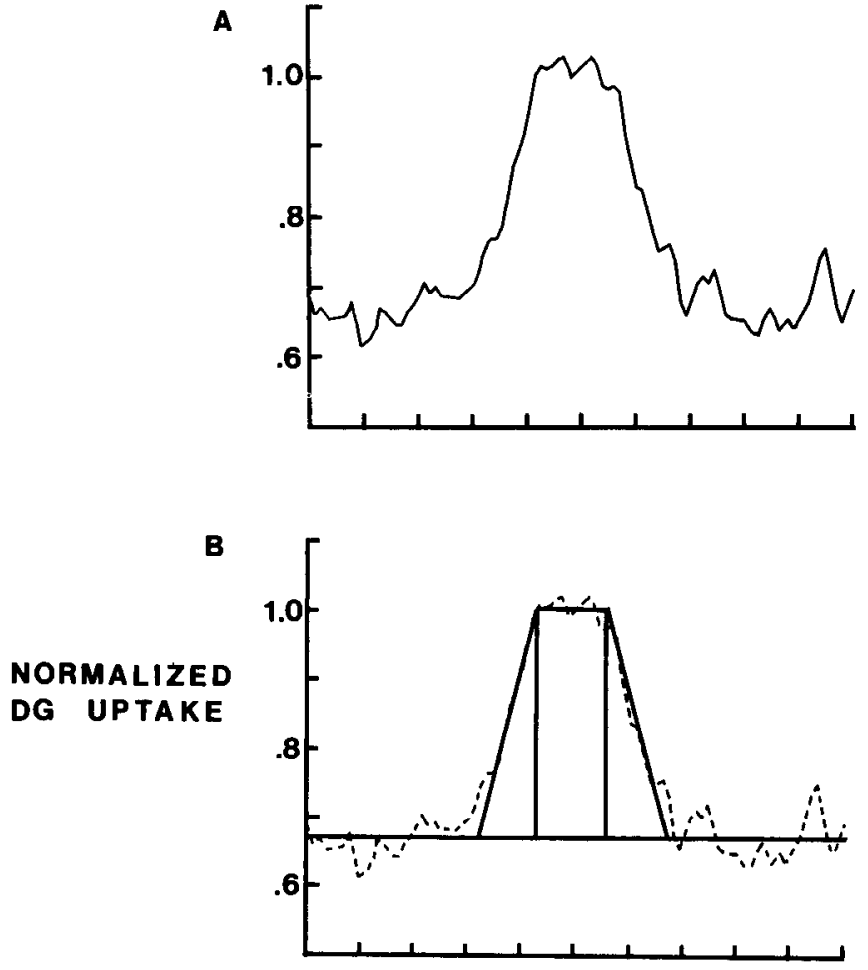

C

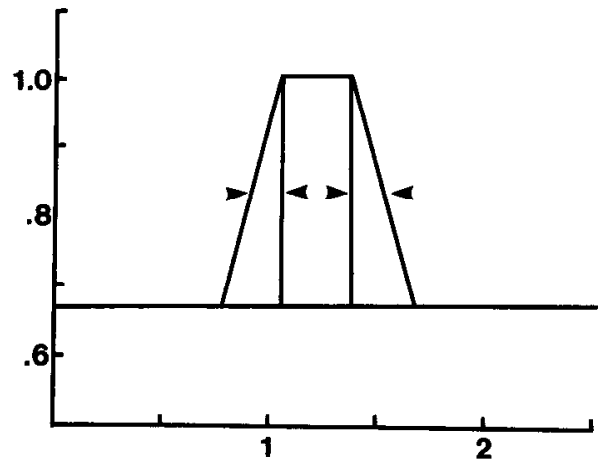

DISTANCE ACROSS CORTEX $(\mathrm{mm})$

Figure 19. Measurement of the sharpness of retinotopic borders from DG autoradiographs. $A$, Levels of uptake for a retinotopically specific segment from layer $4 \mathrm{Cb}$ of the point image case illustrated in Figure 18. The segment was measured parallel to the long axis of the ocular dominance segments, so that the falloffs on the left and right sides of the hump in $A$ reflect the specificity of retinotopic (rather than ocular dominance) organization. In $B$, the DG pattern (dashed lines) is approximated with a series of solid lines indicating baseline uptake, the width of the stimulus check representation, and the slopes of the DG falloff on each side. In $C$. this linear approximation is displayed alone. The half-amplitude at half-width of the DG falloff on each side of the DG "hump" (between arrowheads) can easily be measured. In this case, the falloff in layer $4 \mathrm{Cb}$ has a width of about $140 \mu \mathrm{m}$.

$\mu \mathrm{m}$, and that in layer 5 and at the $2+3$ border was difficult to measure, but was about $500 \mu \mathrm{m}$. The falloff could not be measured in layer 6 and upper layer 2 of the point image case.

\section{Minimum non-overlap}

As described earlier, experimental measurements of the cortical point image may not be equivalent to those of the minimum non-overlap distance. In the present study, DG measurements of the minimum non-overlap distance were made by visually stimulating wide areas of the visual field, while superimposing thin iso-eccentric rings of a mean luminance gray (of varied width) on that stimulated background. In this way, we could examine the horizontal spread of neural activity produced by different background stimuli.

There is reason to believe that the minimum non-overlap distance varies as a function of as many as 4 different variables: (1) eccentricity, (2) lamina, (3) receptive-field size/spatial frequency tuning, and (4) orientation. In the interests of efficiency, we therefore designed a stimulus to reveal variations in each of these 4 variables within a single animal (see Fig. 20A). The stimulus was divided into $490^{\circ}$ quadrants. In 2 nonadjacent quadrants (straddling the vertical meridian), a sinusoidal lowspatial-frequency grating $(0.7 \mathrm{cycle} / \mathrm{deg})$ was presented, and in the other 2, a grating chosen from the middle-to-high-spatialfrequency range $(4.7 \mathrm{cycles} / \mathrm{deg})$. The orientation of the grating in each quadrant was kept constant at an oblique orientation. The gratings were moved across the screen at velocities of $0.6-$ $6 \mathrm{deg} / \mathrm{sec}$. Superimposed on this 4-sector stimulus was a set of 4 solid gray rings ("blankout" rings) of a mean luminance equal to that of the background stimulus pattern. The width of the rings was scaled with eccentricity, so as to be approximately equal in size on the striate cortex $\left(0.17^{\circ}\right.$ at $1^{\circ}, 0.22^{\circ}$ at $1.78^{\circ}$, $0.32^{\circ}$ at $3.16^{\circ}$, and $0.49^{\circ}$ at $5.5^{\circ}$ ) (see Fig. $20 \mathrm{~A}$ ).

The rationale for this rather complicated stimulus is as follows: the low- and middle-high-spatial-frequency quadrants were used to selectively stimulate cells with large and small receptive fields, respectively. Receptive fields that are larger should "fill in" the blankout rings to a greater extent than do receptive fields that are smaller. By selecting the width of the solid gray rings appropriately, it should therefore be possible to produce autoradiographic instances in which a blankout ring representation is visible across the middle-high-frequency quadrant, but is filled in (or blurrier) in the adjacent low-frequency quadrant at the same eccentricity. In order to separate out DG variations in the receptive-ficld width (spatial frequency) dimension from those occurring in the receptive-field length dimension, we kept orientation constant. Thus, in every $90^{\circ}$ stimulus quadrant, the grating ran parallel with the rings (tapping the spatial frequency dimension) at one boundary, and perpendicular to the rings (tapping the receptive-field length dimension) at the other. Finally, by using rings of equal cortical size (instead of equal stimulus size), it was much easier to visualize any differences in the minimum non-overlap distances with eccentricity. Laminar differences could of course be studied by examining the ring representation in different layers.

The overall topography from layers 2 and 3 of this case is shown in Figure $20 B$. A major difference in overlap distance with spatial frequency can readily be seen by comparing the "blankout ring" representations at the transitions from low-tohigh-spatial-frequency regions, in the upper left and middle portions of Figure $20 B$ (respectively). At this border, both the lowand high-frequency gratings run parallel to the rings. Thus one might expect a greater fill-in in the low-spatial-frequency region than in the high-spatial-frequency region, and this is clearly the result obtained. The greater fill-in of the low-spatial-frequency region is found in every layer (see below). In layers $2+3$, the third and fourth rings are unmistakable in the high-spatial-frequency region, but in the low-spatial-frequency region the third ring is completely filled in by neural activity, and the fourth ring 
is only marginally identifiable. The increased fill-in of the lowfrequency region (relative to the high) is shown in Figure 21 for several layers of the third ring representation.

In Figure 20, one can also see a frequency-specific $D G$ pattern we did not expect to find. In the low-frequency quadrants in both hemispheres, rings near the foveal representation produced higher, rather than lower, levels of DG uptake (arrowheads, Figure $20 B$ ). However, this occurred only in layers $2+3$, in the low-spatial-frequency quadrants, and in the rings closest to the fovea. Thus our interpretation of the fill-in differences in the low- and high-spatial-frequency regions is unaffected over most of striate cortex. This unexpected effect was most obvious where the grating orientation ran perpendicular to the rings. On close examination, these wide, "single" strips can instead be interpreted as the sum of 2 closely adjacent strips of high uptake, separated by a lighter region in the midperipheral strips (see most peripheral arrowheads, Fig. 20B). The transition of the low-frequency blankout ring topography from "single strip" to "double strip" to "filled-in with increasing eccentricity" may be another manifestation of an increased minimum non-overlap distance towards the fovea (see below). Of the several upperlayer DG specializations (see Tootell et al., 1988a), this is one of the most puzzling. However, there are a number of parallels between the optimal stimuli for these anomalous DG results and that for certain psychophysical effects (e.g., McCourt, 1982).

In the middle-to-high-spatial-frequency region, it was possible to measure the width of the ring representation at all 4 eccentricities. Measurements were made by means of densitometric scans perpendicular to the ring in layer $4 \mathrm{Cb}$. Layer $4 \mathrm{Cb}$ was chosen for analysis because the DG retinotopic borders in this layer are sharper than in any other layer and the contrast is also higher. Optical density measurements were then converted to levels of DG uptake by using standards. Within any given ring, the width appeared to vary according to the local orientation of ocular dominance strips, in the manner described earlier. Therefore all measurements were made where the ring ran near-parallel to adjacent ocular dominance strips.

Figure 22 shows representative scans from each of the 4 different rings through layer $4 \mathrm{Cb}$. There are some interpolative difficulties in arriving at an exact figure for the DG falloff at each eccentricity, but with a grating of $4.7 \mathrm{cycles} / \mathrm{deg}$, the $\mathrm{min}-$ imum non-overlap distances vary by a factor of perhaps 3 or so, between $1^{\circ}$ and $5.5^{\circ}$ (see Fig. 22). This can be verified by inspection of the first, the third, and the fourth rings in layer 3 of the mid-high-frcquency quadrant (scc Fig. 20B). The fourth ring is quite discrete, the third ring is beginning to be filled in, and the first ring is almost completely filled in.

For comparison, similar measurements of DG falloff in $4 \mathrm{Cb}$ were made at corresponding eccentricities in the cortical point image case (e.g., Fig. 18; case 13). The cortical point image measurements also increased from $5^{\circ}$ towards the fovea, but not nearly so much as in the minimum non-overlap case. In the cortical point image case, the retinotopic borders near the fovea were less than twice as diffuse as those seen in the minimum non-overlap case (Fig. 22; case 44). Possible reasons for this difference are taken up in the Discussion.

The differences in the minimum non-overlap distance with spatial frequency and eccentricity occurred in all cortical layers. However, even within a single spatial frequency quadrant and at a single eccentricity, there is significant laminar variation in the sharpness of the retinotopic borders (Fig. 23). We could analyze border falloffs only in the high-spatial-frequency region, because the blankout ring was partially or completely filled in in all layers of the low-spatial-frequency region (see Fig. 21).

This description of laminar differences in Figure 23 is most enlightening when compared with corresponding laminar differences in the actively stimulated rings of the cortical point image case (see Fig. 18). As with the actively stimulated ring (Fig. 18), the blankout ring border is most clearly defined in layer $4 \mathrm{Ch}$, and it has considerably more spread (in this case, it is almost completely filled in) in layer $4 \mathrm{Ca}$ (Fig. 23). This supports other DG evidence indicating an increased spread of activity within layer $4 \mathrm{Ca}$ relative to that in layer $4 \mathrm{Cb}$. As in the actively stimulated ring case (Fig. 18), layer 3 of the blankout ring case is defined fairly sharply, as if reflecting the retinotopic organization of inputs from parvocellular-dominated layer $4 \mathrm{Cb}$ rather than from the less discrete, magnocellular-influenced $4 \mathrm{Ca}$. This is further evidence against a strong functional projection from $4 \mathrm{Ca}$ to the upper layers (see Tootell et al., 1988c).

In other layers, the retinotopic border results from the point image and the minimum non-overlap (blankout ring) cases are somewhat different from one another. Unlike the results from the point image case, the blankout ring representation in layer 4B appears to be somewhat more discrete than that in underlying layer $4 \mathrm{Ca}$ (see Fig. 23). In layer 2, the blankout ring becomes fairly well filled in (Fig. 23): this may or may not be analogous to the lack of an actively stimulated ring segment in layer 2 (see Fig. 18). In layer 5 (Fig. 23), the blankout ring representation is diffuse, although it is not nearly as diffuse as the actively stimulated ring representation shown in Figure 18. In layer 6 (Fig. 23), the borders of the blankout ring are fairly sharp, although actively stimulated ring segments are completely absent from layer 6 (Fig. 18) at corresponding eccentricities.

Surprisingly, the width of the blankout DG patterns did not vary depending on the local orientation of the grating relative to the orientation of the ring. Even in layers $5+6$ [where receptive fields are reportedly elongated (Gilbert, 1977; Livingstone and Hubel, 1984b)], there was no variation in ring width with local orientation. Possible explanations for this result are discussed below.

\section{Retinotopic spread and acuity limits}

The retinotopic borders illustrated in Figures 18 and 23 seemed quite sharp. We naturally wondered how sharp the borders were relative to psychophysical measurements of acuity and hyperacuity. [In this exercise we do not assume that acuity and hypcracuity mechanisms are necessarily related in striate cortex, since they appear to be quite different mechanisms in psychophysical tests (e.g., Westheimer, 1979).]

To relate the DG and acuity limits quantitatively, we first refer back to the overall CMF of the monkey in which the falloffs were measured (see Fig. 19). Along an oblique ray at $3^{\circ}$, the CMF of this particular monkey was $3.85 \mathrm{~mm} / \mathrm{deg}$ (the average of horizontal and vertical CMFs). At this magnification, 140 $\mu \mathrm{m}$ of cortex (the half-amplitude of $\mathrm{DG}$ uptake in layer $4 \mathrm{Cb}$ ) would correspond to $2.2^{\prime}$ of arc $\left(0.36^{\circ}\right)$.

To estimate the acuity and hyperacuity of the monkey at a comparable eccentricity, we have extrapolated from the human psychophysical data of Westheimer (1982). This is a fairly safe extrapolation, since the foveal acuity and stereoacuity of the monkey are very close to those of man (Cowey and Ellis, 1967; Bough, 1970; De Valois et al., 1974a, b; Sarmiento, 1975). At $3^{\circ}$ eccentricity, the average acuity (minimum angle of resolution) of 2 well-trained observers was about $1.5^{\prime}\left(0.025^{\circ}\right)$, and the 

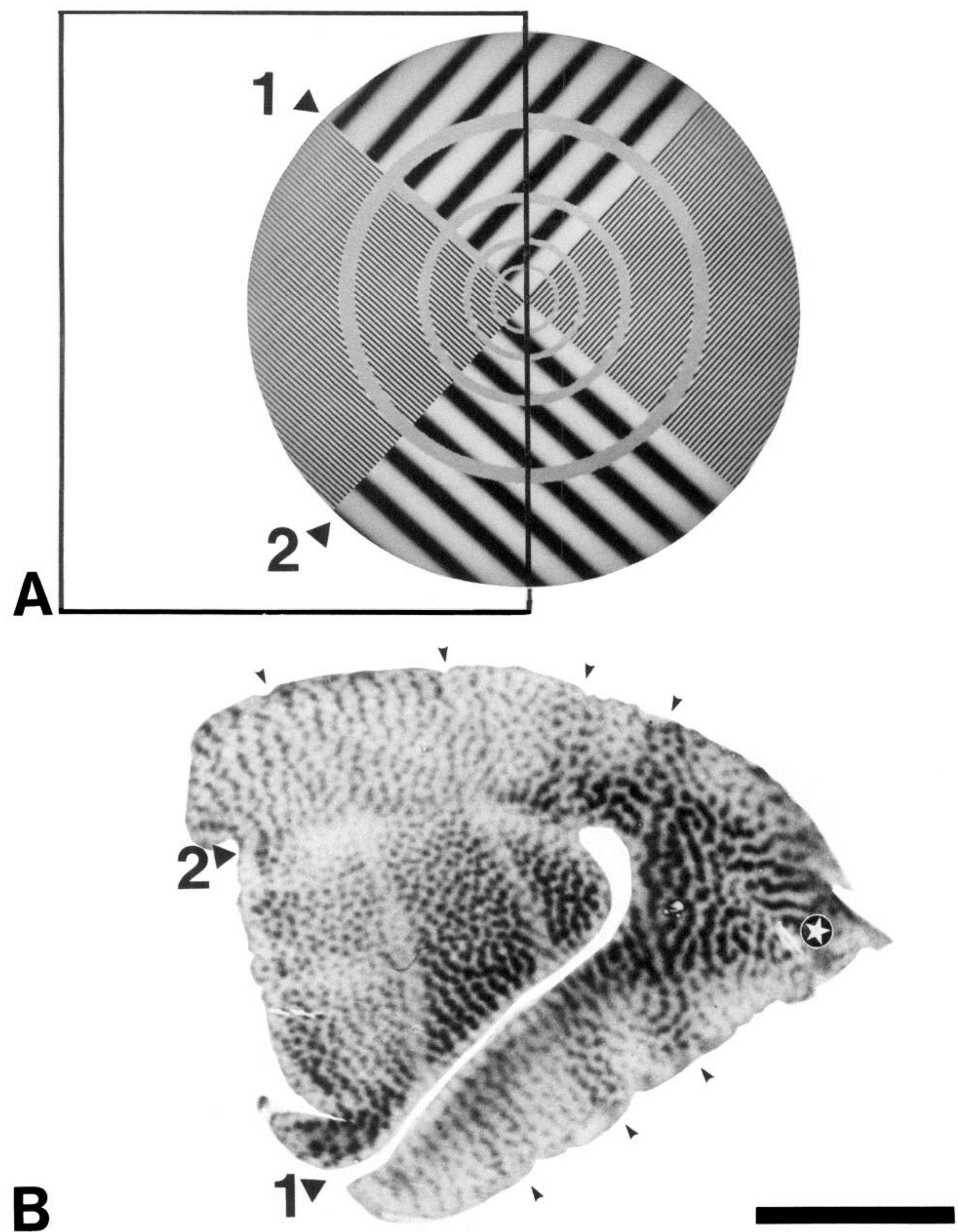

Figure 20. DG analysis of the minimum non-overlap distance. The stimulus is shown in $A$, and the autoradiographic result of the stimulus in striate layer 3 in $B$. The stimulus contained either a low- $(0.7 \mathrm{cycle} / \mathrm{deg})$ or a medium-high $(4.7 \mathrm{cycle} / \mathrm{deg})$-spatial-frequency grating in each of 4 segments. The gratings were moved back and forth along an axis perpendicular to the long axis of the grating stripes, and did not change orientation. Superimposed on this pattern were 4 rings of uniform gray. The width of the rings varied inversely with the striate magnification factor so as to be equally wide in cortex. Further details of (and rationale for) the stimulus are found in the text. The correspondence between the borders of lowand medium-high-spatial-frequency gratings in $A$ (and their corresponding representation in $B$ ) is indicated by the 1 and 2 arrowheads on the left. The fovea is represented at the tip of the operculum (star on the right). The most peripheral portions of the stimulus are represented towards the 

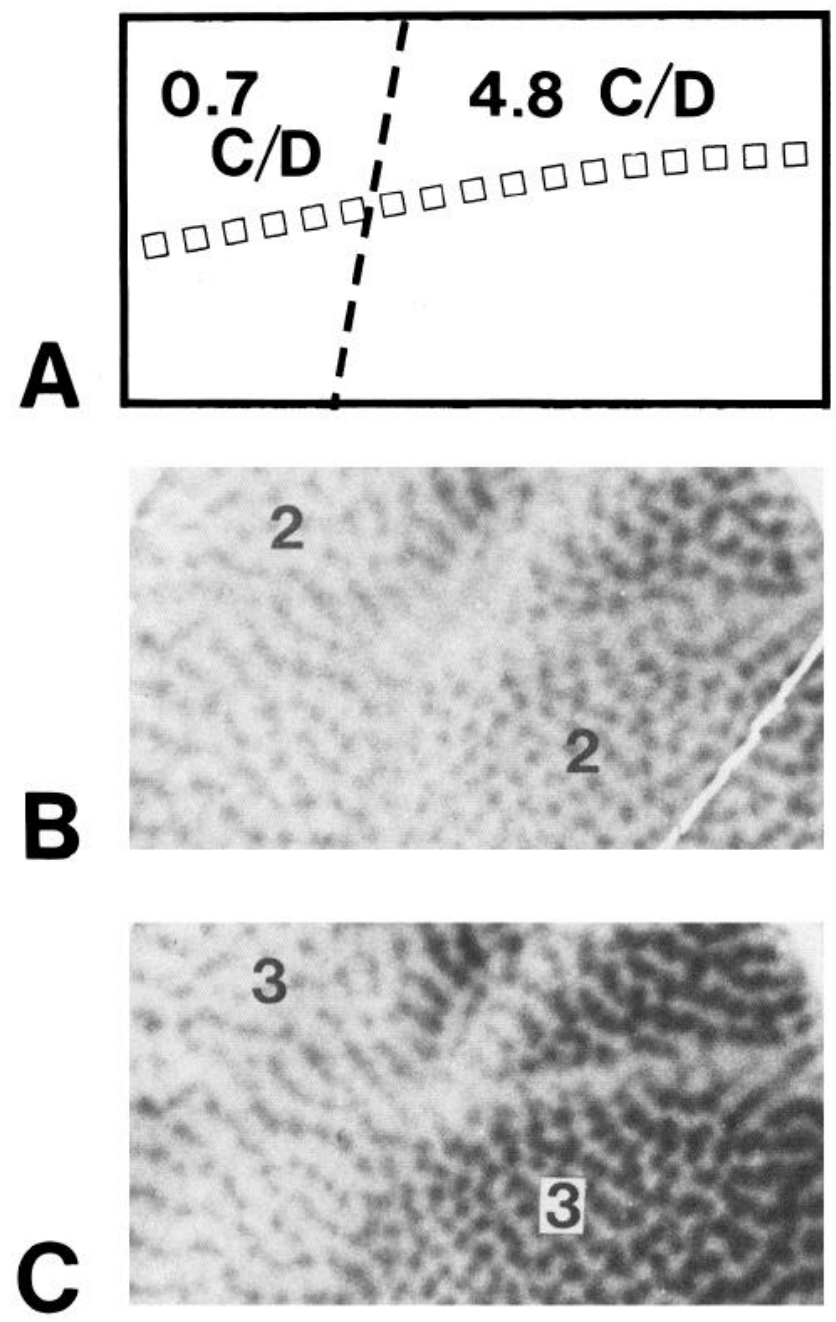
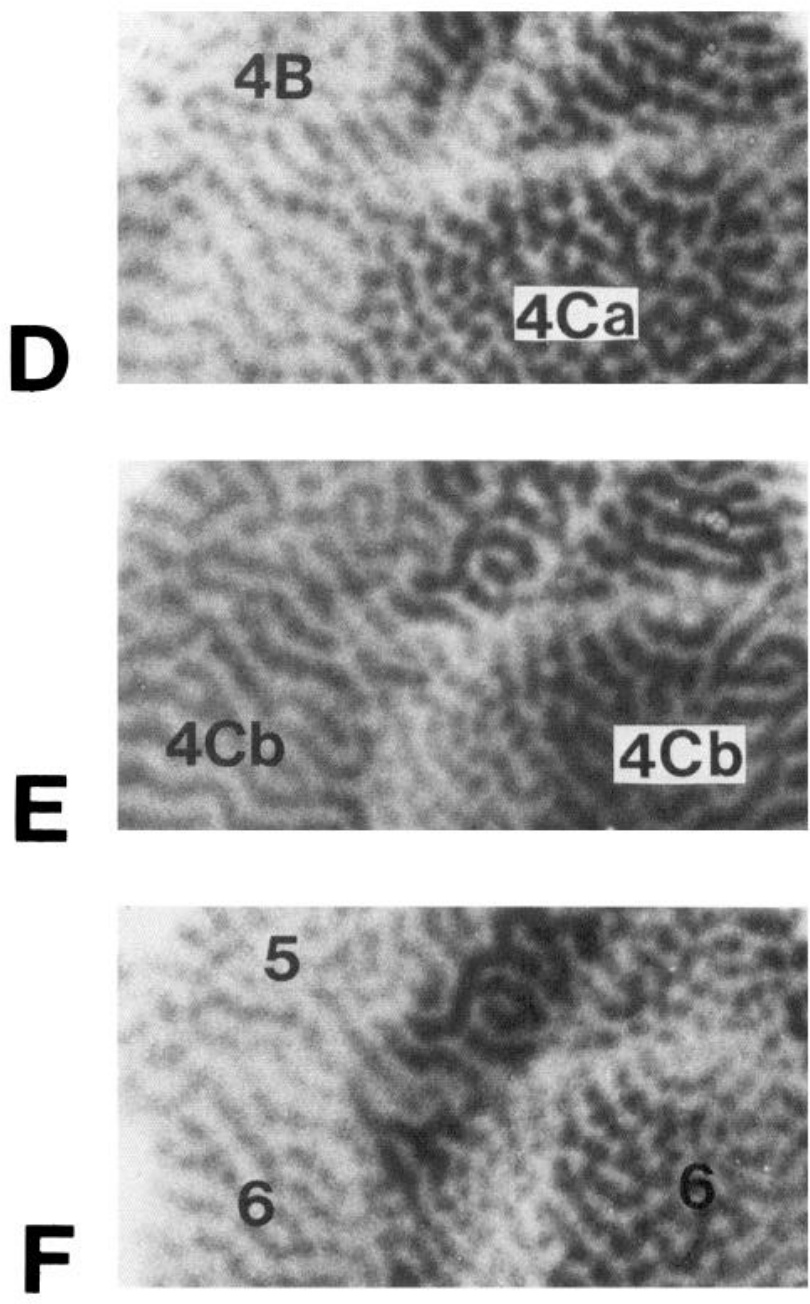

Figure 21. Differences in the effect of background spatial frequency sensitivity on the DG representation of a stimulus blankout ring. The autoradiographs in $B-F$ were taken from topographically corresponding regions of successively deeper sections from the next-to-most-peripheral ring of the case shown in Figure 20 and described in the text. As diagrammed in $A$, the ring was surrounded by a low ( 0.7 cycle/deg)-spatialfrequency grating in the left third of $B-F$, and by a higher ( 4.8 cycle/deg)-spatial-frequency grating in the right-hand portion. The position of the stimulus ring in $B-F$ is represented by a string of unfilled squares in $A$. Representative portions of the different layers are indicated in $B-F$. $B$ is taken from layer 2, and $C$ from layer 3. $D$ includes large regions of layers $4 \mathrm{~B}$ and $4 \mathrm{Ca}, E$ includes a lot of $4 \mathrm{Cb}$, and $F$ includes layer 6, with some 5 and $4 \mathrm{Cb}$. In all layers, the ring representation is more filled in within the low-spatial-frequency background region than within the higher-spatialfrequency background. This indicates that the greater functional overlap of cells tuned to low spatial frequencies is not a peculiarity of layer 3 . Scale bar, $5 \mathrm{~mm}$.

average hyperacuity threshold was about $0.4^{\prime}\left(0.007^{\circ}\right)$ (Westheimer, 1982). The visual angle represented by the half-amplitude falloff of DG uptake in layer $4 \mathrm{Cb}\left(2.2^{\prime}\right)$ is thus very close to the classical acuity limit $\left(1.5^{\prime}\right)$ and about 5 times the hyperacuity threshold.

\section{Retintotopic organization and orientation tuning}

The experiments illustrated in Figures 18 and 23 demonstrate some architectural interactions related to retinotopic position and spatial frequency tuning, but little about the retinotopic

\footnotetext{
left. The intersection of all 4 ring representations with the dorsal V1-V2 border is indicated at the top by small arrowheads. The analogous intersection of the 3 most central rings with the ventral V1-V2 border appears at the bottom. One significant result is that the DG representation of the more peripheral gray rings is clearly visible when a grating of mid-high spatial frequency is used as background, but is filled in when a lowspatial-frequency grating is used. This and other evidence suggests that cells selective for low spatial frequency (presumably having larger receptive fields) arborize over a larger cortical distance than cells tuned to higher spatial frequencies (with smaller receptive fields). An increased fill-in of the DG representation also occurs towards the fovea in the region stimulated by the $4.7 \mathrm{cycle} / \mathrm{deg}$ grating: the representation of the ring closest to the fovea can barely be discerned. This may confirm reports that the cortical point image increases from $5^{\circ}$ to the foveal representation, but other explanations are possible. Unexpectedly, the most foveal rings produce enhanced DG uptake when flanked by a low-spatial-frequency grating. Scale bar, $1 \mathrm{~cm}$.
} 
ECCEN:

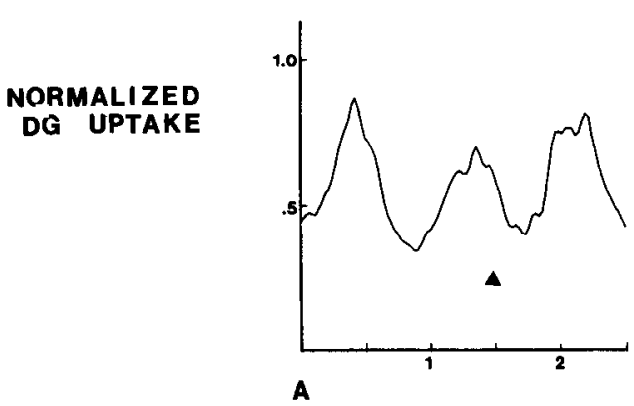

$1.78^{\circ}$

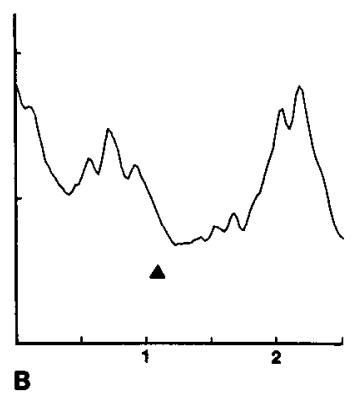

$3.16^{\circ}$

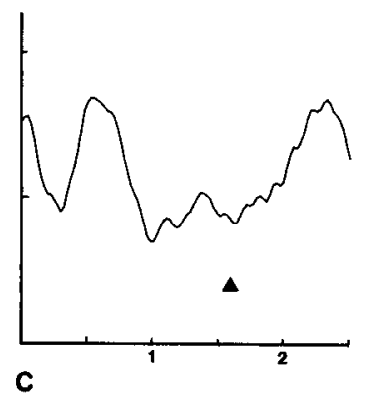

$5.50^{\circ}$

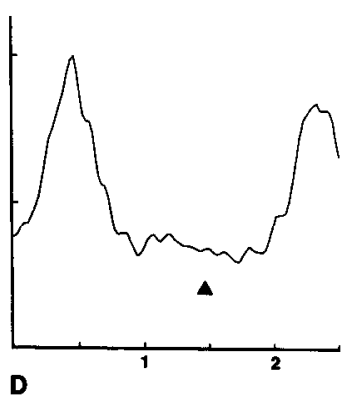

\section{DISTANCE ACROSS CORTEX $(\mathrm{mm})$}

Figure 22. Densitometric analysis of variations with eccentricity in one DG measure of the minimum non-overlap distance. The plots in $A-D$ are all taken from layer $4 \mathrm{Cb}$ of the case illustrated in Figure 20 , at different eccentricities. The plots are taken perpendicular to the cortical representation of a gray blankout ring surrounded by a $4.7 \mathrm{cycle} / \mathrm{deg}$ grating from positions where the ocular dominance strips generally run parallel to the ring representations. Thus the X-axes run roughly perpendicular to the long axes of the ocular dominance strips. The 2 or 3 large humps in each plot therefore indicate higher deoxyglucose uptake in the visually stimulated ocular dominance strips. Each filled triangle indicates the approximate center of the DG blankout ring representation, as judged from densitized views of the whole flattened section. Clearly, the ring representation is more "filled in" towards the foveal representation.

constraints involved in the wiring of orientation tuning. However, another experiment (described briefly above and illustrated in Fig. 7) was specifically designed to look at the spread of orientation-specific activity across cortex (case 52). This measure is significant, because electrophysiological studies have demonstrated that orientation-specific activity may be linked across several millimeters of cortex (Ts'o et al., 1983). Such a spread of activity would seem greater than that demonstrated in the point image or nonoverlap cases in any layers of macaque parafoveal striate cortex. Furthermore, since DG uptake in retinotopically specific single-orientation cases also has a distinctive laminar profile, it is possible that the spread of orientationspecific activity might differ in layers other than those seen in the cortical point image tests (see Figs. 18, 19).

In this experiment, a stationary, horizontal square wave was shown to a monkey monocularly, and the stimulus was counterphased against a steady gray grating. This produced a DG pattern of roughly parallel lines that diverged as the foveal representation was approached (Fig. 7). The overall contrast of the DG pattern was fairly low, as in all the counterphased, orientation-specific cases we have seen. (A similar "preference" for moving rather than counterphased stimuli is seen in many striate single units.)

In Figure 24, one can see a comparison of the topography in different layers, ranging from about $2^{\circ}$ to $6^{\circ}$ eccentricity. We assume that horizontal-preferring cells at the representation of the edges of the stimulus stripes were selectively stimulated in this case. This conclusion is based on several "orientation-specific" aspects of the DG results, as well as on what is known about striate single-unit responses. For instance, in other studies using moving bars at a single orientation, DG uptake is highest in layers $4 \mathrm{Ca}$ and 6 (Hubel et al., 1978; Livingstone and Hubel, 1984a). Such a distinctive laminar "signature" for orientationspecific stimuli can also be seen in this case (Fig. 24); this supports our assumption that the isoelevation "lines" of high DG uptake are orientation-specific as well as retinotopically-specific.

Other evidence also supports this idea. In studies using moving bars or gratings, DG orientation columns (Hubel et al., 1978) and orientation-selective cells (Hubel and Wiesel, 1968, 1977; Blasdel and Fitzpatrick, 1984) have not been found in layer
$4 \mathrm{Cb}$. Thus, one might expect a pattern of DG uptake in layer $4 \mathrm{Cb}$ of this case that is continuous throughout the extent of the stimulated ocular dominance strips. At peripheral eccentricities, such a striplike topography can be demonstrated in $4 \mathrm{Cb}$ (see Fig. 23). In $4 \mathrm{Ca}$, the pattern of DG uptake in the orientationspecific stimulus borders is instead more spotty, as one would expect from the intersection of ocular dominance and orientation columns, both of which are found in this layer (Blasdel and Fitzpatrick, 1984; Livingstone and Hubel, 1984a). The ocular dominance-retinotopic stripe topography is simiarly broken up by orientation columns in all layers except $4 \mathrm{~A}$ and $4 \mathrm{Cb}$. DG orientation columns are also weak or absent in layers $4 \mathrm{~A}$ and $4 \mathrm{Cb}$ when produced by moving, orientation-specific gratings (see Hubel et al., 1978, and unpublished results). Thus, the laminar pattern of DG uptake in these geniculorecipient layers is roughly what one would expect from predominant DG uptake due to orientation-specific mechanisms within most layers of striate cortex and to nonoriented cells in $4 \mathrm{~A}$ and $4 \mathrm{Cb}$.

In striate layers $4 \mathrm{Ca}$ and 6 , the orientation-specific DG topography produced by the stripe borders spreads out much further in the horizontal direction than it does in layers 3, 4A, and $4 \mathrm{Cb}$. For instance, in the lower middle of Figure 24D, the DG periodicities form an almost continuous sheet without obvious evidence of modulation by the stimulus stripe edges. In topographically identical portions of layer 3 (Fig. 24B) or layer 4A (Fig. 24C), the representation of the stimulus stripe edges can be easily resolved. The spread in layers $2,4 \mathrm{~B}$, and 5 is intermediate in extent. Although it is difficult to quantify the maximal extent of the horizontal spread of activity in these layers, it appears to be greater than that seen earlier in the point image and minimum non-overlap cases: half-amplitudes in some layers seem to be greater than a millimeter.

Levels of stimulus-related uptake are much higher in the magnorecipient layer $4 \mathrm{Ca}$ than in the parvorecipient layer $4 \mathrm{Cb}$. In fact, towards the fovea, stimulus-related uptake in layer $4 \mathrm{Cb}$ becomes vanishingly faint (Fig. 24E), although the strip representation is clearly visible in $4 \mathrm{Ca}$ at all eccentricities. Increasingly, results in layers outside layer $4 \mathrm{C}$ do not appear to differ according to the presence or absence of stimulus-driven uptake in underlying layer $4 \mathrm{Cb}$. This fact (coupled with the presence 


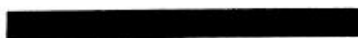

\section{$5 \mathrm{~mm}$}
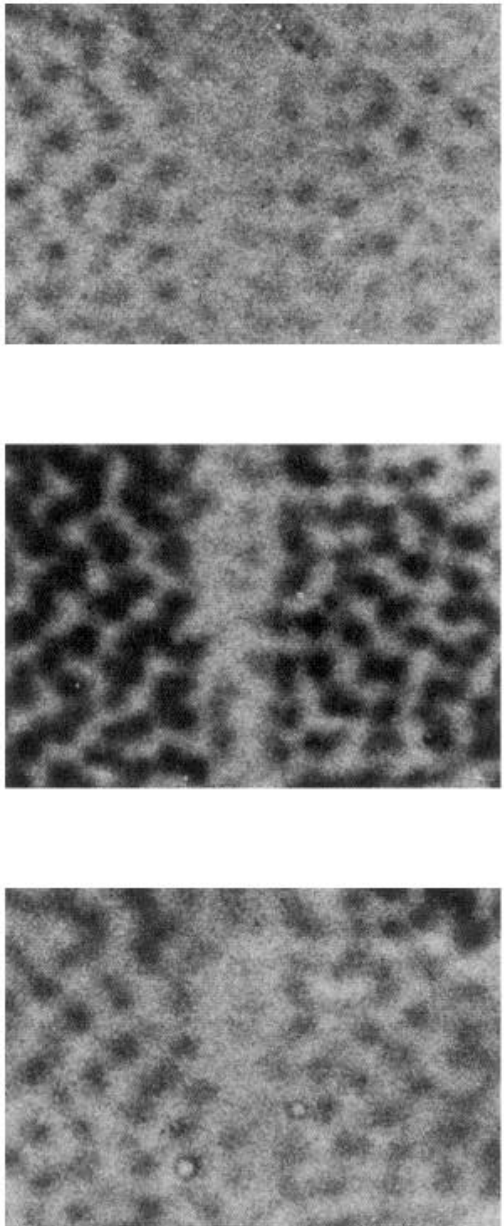

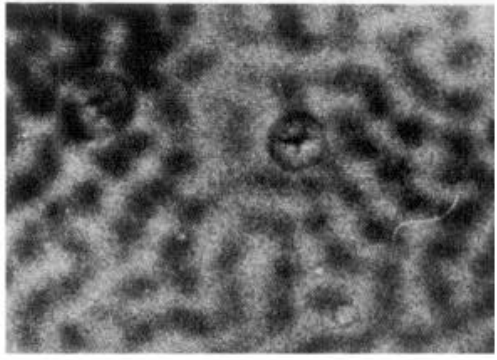

$4 \mathrm{Ca}$

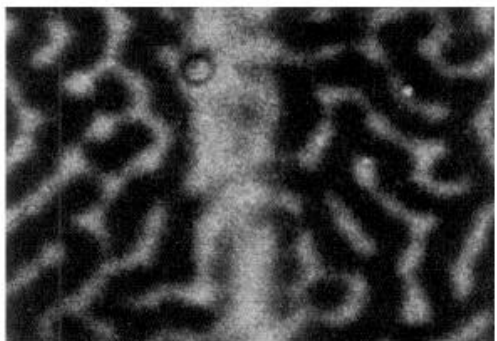

$4 \mathrm{Cb}$

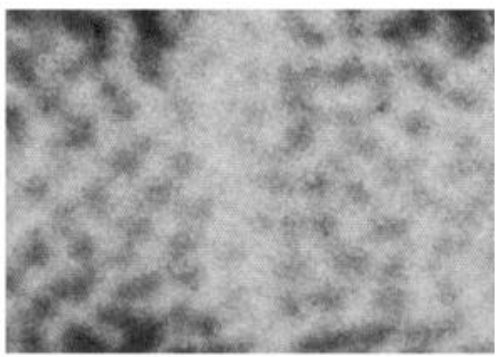

5
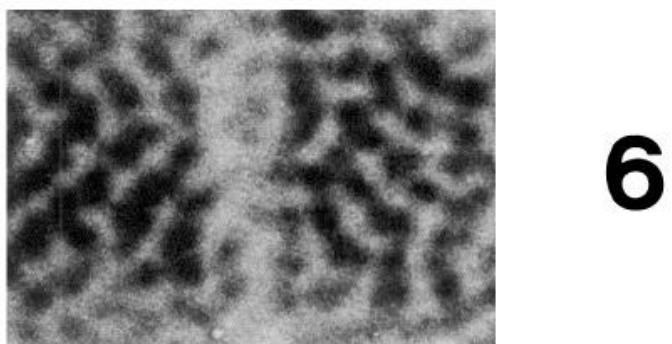

Figure 23. Laminar variations in the representation of a DG blankout ring. The stimulus represented in each panel was a uniform gray ring $\left(0.5^{\circ}\right.$ wide) at an eccentricity of $5.5^{\circ}$, surrounded by a high-contrast grating of 4.7 cycles/deg. Each section represents a single ring in topographically corresponding portions of different layers. The ring representation is quite discrete in layer $4 \mathrm{Cb}$, and much more filled in (along the long axis of the ocular dominance strips) in layer $4 \mathrm{Ca}$. These differences in retinotopic resolution between the magnocellular-recipient layer $4 \mathrm{Ca}$ and the parvocellular-recipient $4 \mathrm{Cb}$ parallel those seen in the point image case (Fig. 18). The ring representation in other layers shows various degrees of fill-in.

of orientation selectivity and orientation columns in magnocellular-recipient layer $4 \mathrm{Ca}$ ) argues for a special role of the magnocellular layers in the wiring of orientation tuning within striate cortex.

\section{Blob domains}

Interlob size and functional activity

The spacing of the cytox blobs and hypercolumns is strikingly regular. Without such regularity, one might expect to find "perceptual holes" within the visual field (Hubel and Wiesel, 1977). For instance, if horiziontal orientation columns were spaced randomly (rather than regularly) throughout cortex, some regions would (by chance) be devoid of horizontal-oriented cells, which might render small horizontal lines invisible in corresponding regions of the visual field.

Although the idea of avoiding perceptual holes has often been suggested as a reason for the regular spacing of hypercolumns and cytox blobs in striate cortex, the converse side of the issue has not been discussed: How regular do the periodicities have to be in order to prevent perceptual holes? In fact, the latter question clarifies the former. Moreover, answers to the latter question fall out of a straightforward analysis of (1) the CMF, 

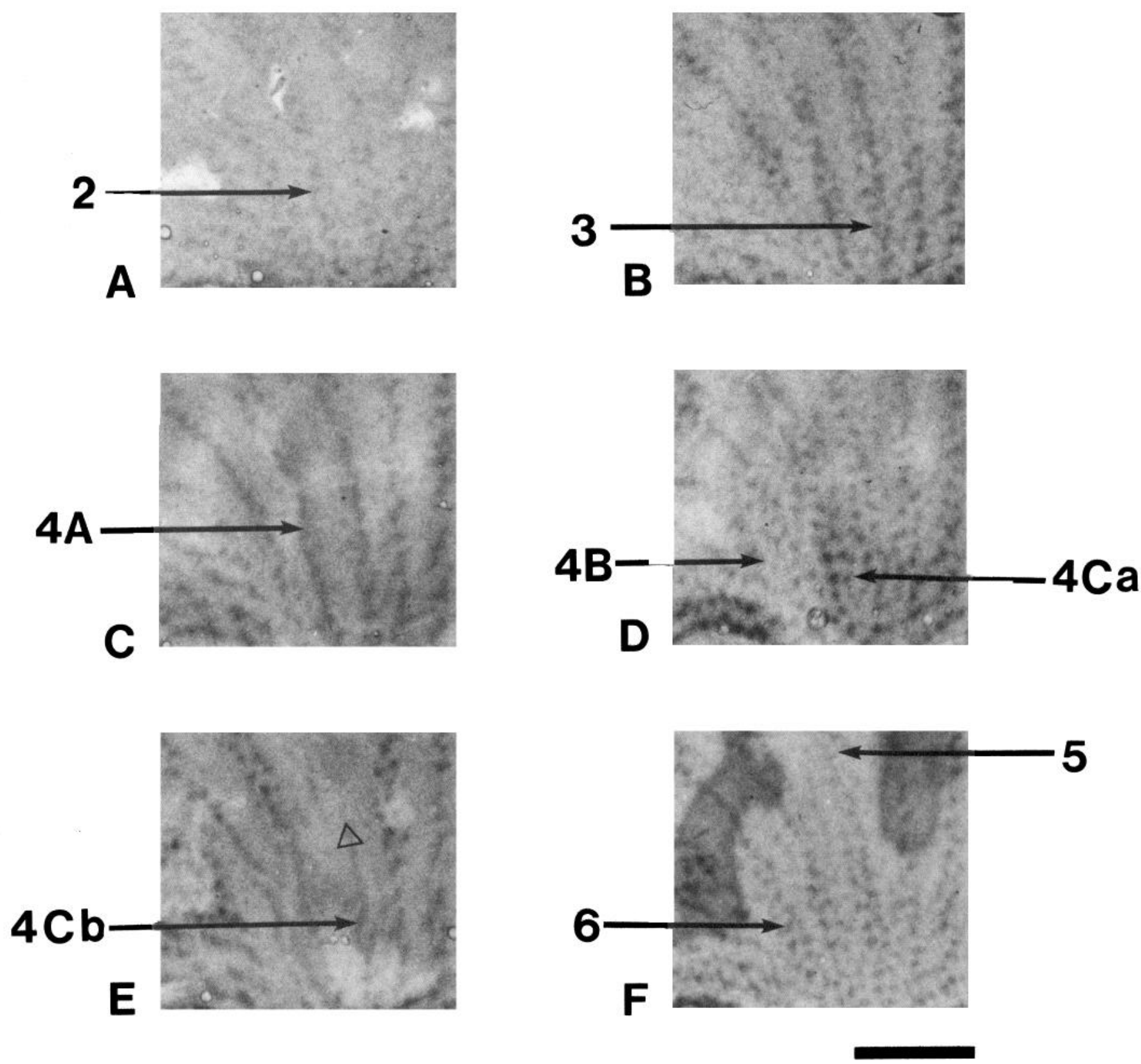

Figure 24. Laminar differences in the DG pattern produced by stationary (counterphased) grating stripe edges. The autoradiographs in $A-F$ are taken from successively deeper sections and topographically corresponding regions of the case described in Figure 7. The peripheral edge of the operculum is represented along the bottom of $A-F$, and the horizontal meridian is represented along the right-hand sides. Several laminar variations are worth noting. Both of the geniculorecipient layers driven directly by the parvocellular layers (layers $4 \mathrm{~A}$ and $4 \mathrm{Cb}, C$ and $E$ ) show striplike regions of DG uptake; presumably these are portions of ocular dominance strips (due to the monocular stimulation conditions) unpermuted by orientation columns (which are absent in $4 \mathrm{Cb}$ and almost absent in $4 \mathrm{~A}$ ). In other layers containing orientation columns (including magnocellular-recipient layer $4 \mathrm{Ca}, D$ ), the DG pattern is broken apart into a spotty array, which presumably represents the intersection of ocular dominance columns and orientation columns. Closer to the fovea, the DG representation of the stripe edges fades out in layer $4 \mathrm{Cb}$ (see the region above the unfilled triangle in $E$ ). However, a stripe representation does occur in all corresponding portions of all other layers; this is presumably driven largely by magnocellular inputs via striate layer $4 \mathrm{Ca}$, since $4 \mathrm{Cb}$ shows no stripe representations at these eccentricities. The representation of the separate stripes in some layers (e.g., 4B, 4Ca, 5, and 6) is so wide that it forms an almost uniform array of DG periodicities at the lower (more peripheral) portion of $D$. In other layers (e.g., layers $4 \mathrm{Cb}, 4 \mathrm{~A}$, and 3), the DG stripe representation is more discrete. Scale bar, $5 \mathrm{~mm}$.

(2) minimum and maximum receptive-field size, and (3) the spacing between cytox blobs within a local striate region. Since data on these parameters were readily available in our DG data, we performed such an analysis.

Since all the hypercolumn topographies are rigidly related to the cytox blob topography (Horton and Hubel, 1981; Tootell et al., 1982b; Horton, 1984), we were interested in estimating the size of the visual-field segment projecting to each blob. However, as we have shown, the horizontal spread of functional activity varies as a function of layer, receptive-field size, eccentricity, etc. Therefore it is somewhat meaningless to estimate "the" visual-field area projecting to each cytox blob domain. 


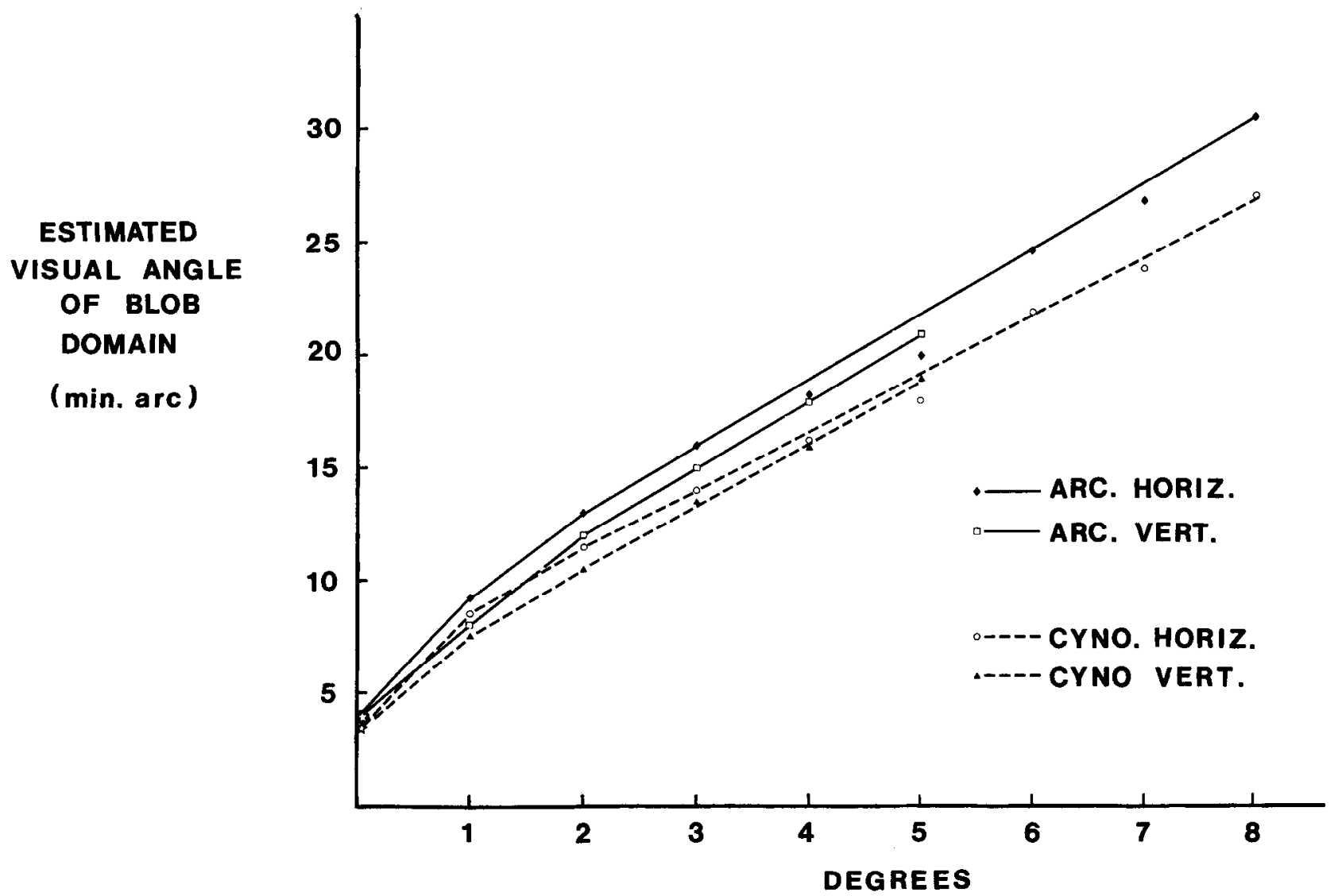

Figure 25. Estimated visual angle subtended by the representation of a blob domain at different eccentricities. A "blob domain" consists of either (1) one blob plus half the surrounding interblob area or (2) twice this cortical area spread over the duplicate representation in blobs from both eyes. The angular distance subtended by each blob definition is equivalent. The angle was computed by direct measurements of the CMF (from DG maps) and of the number of blobs in counts from the same and similar tissue. Data from $M$. arctoides are indicated by solid lines, and data from $M$. fascicularis (cynomologous) are indicated by dashed lines. Computations along the vertical and horizontal meridians differ slightly, because the ocular dominance strips run near-perpendicular relative to the vertical meridian, and more randomly relative to the horizontal meridian (see text). Scale bar, $5 \mathrm{~mm}$.

Instead, we estimate the visual-field area corresponding to the distance between the centers of adjacent cytox blobs. Such a figure can be specified exactly.

First, all thc blobs were counted on 7 randomly chosen operculae, and each total was divided by the area over which blobs were counted. This yielded an average interblob spacing of 490 $\mu \mathrm{m}$ for $M$. fascicularis and $M$. assamensis, and of $550 \mu \mathrm{m}$ for the larger $M$. arctoides. (In this analysis, the elongation of blob spacing that often occurs parallel with ocular dominance strips has been averaged out.) To compute the visual angle corresponding to the distance between blobs, the $\mathrm{CMF}^{-1}$ from Figure 9 was multiplied by the average interblob spacing, at $1^{\circ}$ intervals over a range from $0^{\circ}$ to $8^{\circ}$ eccentricity.

The visual field was duplicated in each ocular dominance strip, and each strip had its own set of cytochrome oxidase blobs. Therefore, an idealized blob domain receiving input from a single portion of the visual field could be modeled either as one cytochrome oxidase blob plus half the surrounding interblob areas or as a pair of cytox blobs in adjacent ocular dominance strips, plus half the interblob area surrounding them. Since the visual field was duplicated, the area of the visual field represented in each of these 2 blob domains was identical. We do not assume that the actual visual-field regions of any 2 blobs will be identical, only that they are equal in size, and, on average, duplicated.

To compensate for the duplication of the visual field in adjacent blobs, we doubled each of the average interblob values obtained above along an axis perpendicular to the ocular dominance strips. For blobs along the vertical meridian, this meant multiplying the obtained values by 2 (since ocular dominance strips generally run perpendicular to the vertical meridian). Along the horizontal meridian, the ocular dominance strips cross the meridian at more random orientations, so values along the horizontal meridian were multiplied by 1.5 . The latter estimate is somewhat arbitrary; therefore, our estimate along the horizontal meridian should be given less credibility than those along the vertical meridian.

These values for the distances between the visual-field centers of adjacent blob modules are plotted in Figure 25. Values along the $Y$-axis are visual angles corresponding to the interblob distance (or to the distance connecting interblob midpoints across a blob) at a given eccentricity. From Figure 25 , it can be seen that the distance between the centers of foveal blobs corresponds to about $4^{\prime}$ of arc, and it increases to about $1 / 2^{\circ}$ at eccentricities of $8^{\circ}$.

From our DG analysis of the minimum non-overlap distance, 
and from single-unit data (Dow et al., 1981; Van Essen et al., 1984), it is apparent that activity from the larger receptive fields in each blob extends over several adjacent cytox blobs (see Figs. 20,21 ). For this subset of cells, the packing density (or the regularity) of the blobs and hypercolumns could be relaxed without creating perceptual holes. The extensive and mutual overlap of these cells makes it likely that they integrate spatial information between different but nearby blob domains.

It is also clear that cells with the smallest receptive fields (and a greater sensitivity to spatial detail) have receptive fields that could be encompassed within a single blob domain. For instance, our sharpest DG falloffs (140 $\mu \mathrm{m}$ half-amplitude) imply a resolution that is quite sharp relative to the size of a blob domain $(\sim 400 \times 600 \mu \mathrm{m})$. Furthermore, a 7 cycle/deg grating produces DG uptake that extends at least as far as $10^{\circ}$ eccentricity. At this eccentricity, one-half cycle (one stripe) of the grating will be mapped across about $100 \mu \mathrm{m}$ of cortex, which corresponds to about a fifth of the diameter of a blob domain. Thus there is some experimental reason to believe in a finegrained retinotopic organization within a blob domain, and it is easy to see how an increased distance (or a decreased regularity of spacing) between blobs could lead to perceptual "holes." As elsewhere in the natural world, the brain may be just as orderly as it needs to be.

At one point we imagined that the number of striate blobs might be rigidly constant across animals. Counts were therefore made of the number of blobs in 6 operculae, and in the whole of striate cortex in both hemispheres of 2 monkeys. (In one monkey, striate cortex was completely unfolded using a different and extensive flattening procedure; Tootell and Silverman, 1985.) From these counts it became obvious that the absolute number of blobs in different animals (even in different hemispheres) was quite variable, but that the total number was greater in the larger $M$. arctoides $(\sim 9000)$ than in $M$. fascicularis $(\sim 6000)$. These estimates tally quite well with the estimates of Horton (1984), although a species difference was not reported in the carlicr study.

\section{Discussion}

The DG data described in the present study furnish a very (perhaps overly) detailed description of the striate retinotopic organization at both the general and local levels. We first discuss the data on the overall retinotopy, and then the data on local striate organization.

\section{Overall retinotopic organization}

In general, the overall retinotopic data are in good agreement with the results from previous electrophysiological studies (see Fig. 11). The average $\mathrm{CMF}^{-1}$ measured in the present study has a slope of 0.066 and $0.052 \mathrm{deg} / \mathrm{mm}$ along the vertical and horizontal meridians, respectively. Several converging lines of DG evidence indicate that the fovea is represented exactly at the rostrolateral region of acute curvature (the "apogee") in the V1$\mathrm{V} 2$ border, and the average magnification at this point is about $16 \mathrm{~mm} / \mathrm{deg}$. There is a certain amount of variation between animals in the cortical magnification, and perhaps in the magnification factor at the fovea as well. This interanimal variation may explain the few minor discrepancies between the data in this DG study and those in some of the previous electrophysiological studies. However, a magnification factor of $30 \mathrm{~mm}$ / deg (as reported by Dow et al., 1981, 1985) appears to be beyond the bounds of variation seen in the present study. Van Essen et al. (1984) reached a similar conclusion.

\section{Overrepresentation/duplication of the vertical meridian}

We presented evidence against both overrepresentation and duplication of the vertical meridian representation in the striate cortical maps. Since there is strong evidence from anatomical studies that a significant duplication (on the order of a degree wide) exists at the retinal level, this poses something of a quandary. It is conceivable that there is a duplication in the projection from retina to LGN, and not from LGN to cortex. However, from a design perspective, the advantages of such a scheme are obscure. Simpler possibilities are that either the HRP or the DG evidence is wrong, or that, for some reason, the anatomical projections to the "wrong" hemisphere are functionally suppressed so that they do not contribute to the DG maps.

\section{Superior and inferior visual fields}

We find a relative overrepresentation of the inferior visual field in the central half of striate cortex, as has been reported by Van Essen et al. (1984) in peripheral striate cortex. The implications of this finding are unknown. However, it may be related to the fact that at least one extrastriate area (V3) appears to serve only the inferior visual field, without a mirror-symmetric counterpart serving the superior visual field (Felleman et al., 1984; Burkhalter et al., 1986). Teleologically, it is possible that the inferior visual field is more important for survival: under most viewing conditions, the superior visual field will be mostly sky, at least in primates living outside of the forest. The overrepresentation of the inferior visual field may originate (at least partly) in the retina (Perry et al., 1984).

\section{Interanimal variability}

Interanimal variations in the cortical magnification factor have been mentioned in most previous papers, and are quantitatively analyzcd in the present study (sce Figs. 12,13) and by Van Essen et al. (1984). In the present study it was possible to show that much of this variation is correlated with the size of striate cortex: larger brains have a retinotopic map that is correspondingly enlarged.

In this analysis we compared retinotopic data in each animal to the distance between specific brain regions; this distance amounted to about half of the length of striate cortex along the horizontal meridian. If the complete area of striate cortex were known in each animal from which retinotopic data were available, such a compensation for brain size might further reduce the variance obtained in retinotopic measurements.

The notion that "the" cortical magnification factor is scaled proportionate to brain size is attractive, because the area of striate cortex differs by a factor of over 2 in different individuals of the same Old World primate species (e.g., Van Essen et al., 1984), and even greater variations in striate area can be seen between different species within the Old World primate order (cf. man vs cercopithecus or macaque). The notion also has some developmental advantages, since brain size in the adult is considerably larger than it was when retinotopic connections were first made.

Without a scaling of magnification factor proportionate to overall striate area, the magnification at the foveal and/or the peripheral representations would, of course, be grossly different in different animals. On the other hand, there is some evidence that Old World monkeys with much smaller brains have slightly 
lower acuities in behavioral tests. The acuity thresholds of human observers slightly exceeded those of Macaca nemestrina, which slightly exceeded those of Macaca fascicularis (De Valois et al., 1974b). In this regard, it is worth noting that Macaca mulatta, which is larger than $M$. fascicularis, also has a peak cone density that is correspondingly higher in the fovea (Borwein et al., 1980; Perry and Cowey, 1985). If we assume that the acuity differences (and differences in peak cone density) are correlated with differences in the magnification factor at the fovea, this finding implies that the scaling of magnification to striate area is less than proportionate to the variation in striate size across species.

\section{Distortion controls}

Our DG data on the overall striate retinotopy are taken from tissue that has been unfolded slightly relative to its shape in vivo. Obviously, any distortion in the flattening procedure will influence the retinotopic data we have obtained. In a very careful modeling study, Merker and Schwartz (1985) have shown that, in principle, the striate operculum can be unfolded without introducing significant distortion, except in the region of the lateral calcarine (ectocalcarine) fissure. However, we have noticed that about $40 \%$ of our animals have no significant lateral calcarine fissure, and in these cases the lateral operculum can presumably be unfolded without significant distortion. The theoretical analysis of Merker and Schwartz (1985) thus accords well with our own empirical measurements of distortion in the flattened operculae. Distortion in each of the measured cases was generally $\pm 5-10 \%$, but it was nonsystematic across cases and thus it averaged out to near zero. We took no retinotopic measurements across the lateral calcarine fissure, so we believe that the major retinotopic effects measured in this study are largely uncontaminated by distortions introduced by flattening.

\section{Ocular dominance and retinotopic organization}

In the present study, we have shown a number of examples in which the monocular DG representation of a given (thin) stimulus, running perpendicular to the ocular dominance strips, was about the same size as the representation of an equally thin stimulus strip running near-parallel to the ocular dominance strips (see Fig. 17, $A-C$ ). Since the visual field is duplicated in striate cortex, this means that the representation of the original stimulus ring is actually about twice as wide perpendicular (rather than parallel) to the ocular dominance strips. By itself, this data would constitute solid evidence for a 1:1 (rather than a 2:1) model of cortical magnification relative to the ocular dominance strips. However, resolution uncertainties inherent in the present DG technique do not allow us to rule out the fainter possibility of a slight $(<2: 1)$ increase in magnification factor perpendicular to the ocular dominance strips, (the ca. 1.5:1 model). Other evidence from more global measurements of the DG retinotopic results (Fig. 17, $D-F$, and 'Tootell et al., 1982a, b) and from quantitative analysis of anisotropies in the mapping of the optic disk (Horton, 1984) also supports the idca of an expansion of the representation perpendicular to the ocular dominance strips, without ruling out the possibility of a minor magnification increase (the $\sim 1.5: 1$ model).

If we assume that there is a significant expansion of the overall retinotopic representation perpendicular to the ocular dominance strips, a number of the features of the ocular dominance topography make sense. First, one common finding in all macaque species is that ocular dominance strips run perpendicular

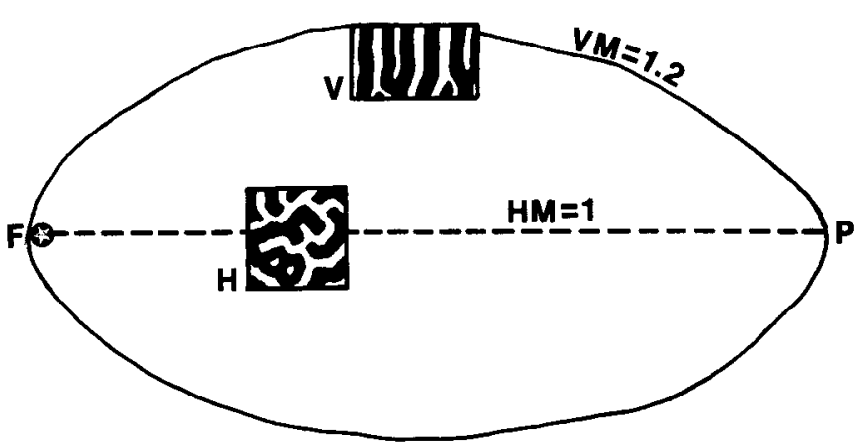

Figure 26. Possible explanation for differences in the orientation of ocular dominance strips near the representations of the horizontal meridia versus those of the vertical meridia. Schematic outline of a flattened, unfolded striate cortex. The fovea is represented at $F$, and the far periphery at $P$. A broken line indicates the position of the horizontal meridian $(H M)$. Owing to the overall shape of striate cortex, it appears that the vertical meridian ( $V M$ ) is longer than the horizontal meridian by a factor of (in this cortex) about 1.2. Knowing this, one might suppose that a patch in the visual field that maps to a square $(H)$ along the horizontal meridian might well be mapped as a rectangle $(V)$ if it appeared instead along the vertical meridian. This is also in accord with experimental results (Van Essen et al., 1984, and present results). It is known from other studies (LeVay et al., 1975, 1985; Tootell et al., 1988a) that ocular dominance columns run in parallel strips perpendicular to the vertical meridian (as in $V$ ), but are much more randomly oriented near the horizontal meridian (as in $H$ ). In the present study, we have shown that there is an expansion of the overall cortical representation perpendicular to the long axis of the ocular dominance srips, because of the duplicated maps in the 2 sets of ocular dominance strips. This rationalizes the differences in orientation of ocular dominance strips: the strips need to run more perpendicular to the vertical meridian than to the horizontal meridian because the map is expanded along the vertical relative to the horizontal meridian. The argument can be just as easily framed in the reverse order: since the map is expanded along the vertical meridian, the strips are forced to run more perpendicular to the vertical meridian than to the horizontal meridian.

to the vertical meridian (along the extent of the V1-V2 border), but that they run much more randomly relative to the horizontal meridian. The representation of the vertical meridian appears to be physically longer than that of the horizontal meridian, and (not surprisingly) the magnification is larger along the vertical than along the horizontal meridian. Thus, the perpendicularity of the ocular dominance strips may serve naturally to expand the map along the vertical meridian. This idea is illustrated fully in Figure 26. There is a similar rationale for the anisotropy in overall retinotopic mapping near the V1-V2 border (Van Essen et al., 1984).

A second idea about the eye dominance anisotropy is more speculative, but suggests an interesting experiment. We showed earlier that the shape of the striate operculum can vary quite a bit among animals (see Fig. 15). Because the topography of ocular dominance strips is also idiosyncratic in different animals, it is possible that the 2 findings are related. Following this hypothesis, opercular striate cortices that are relatively "fat" (such as that in Fig. 15A) should be found to have ocular dominance strips running relatively parallel to the horizontal and vertical meridia. Likewise, "thinner" striate cortices (such as that shown in Fig. 15B) should be found to have ocular dominance strips running more perpendicular to the horizontal and vertical meridia.

\section{Spread of physiological activity}

In previous electrophysiological results (Hubel et al., 1974; Hubel and Wiesel, 1977; Dow et al., 1981; Blasdel and Fitzpatrick, 
1984; Van Essen et al., 1984), and in results from the present study, it has become abundantly clear that the spread of physiological activity varies as a function of several interrelated variables. Each of these is described in turn below.

\section{Spatial frequency tuning/receptive-field size}

Electrophysiological measurements of the spread of activity have often been couched in terms of average receptive-field size, or of the distance between receptive fields of average size. Recently, DG and electrophysiological evidence has appeared that suggests that cells with lower- or higher-spatial-frequency tuning (which are associated with larger and smaller receptive-field sizes, respectively) are grouped into subdivisions within a given layer (Tootell et al., 1981, 1982a, 1983a, 1988c, d; Silverman, 1984). Thus, if one is inadvertently recording from a cell group with lower-spatial-frequency tuning, then the estimates of receptive-field non-overlap distance will be commensurately inflated. By the same token, if one is recording from a cell group with relatively high spatial frequency tuning, then estimates may be artifactually decreased.

In order to test these ideas, we examined the amount of DG "fill-in" within the representation of a thin gray ring when surrounded by either a low- or a high-spatial-frequency grating. (The low- and high-spatial-frequency gratings selectively stimulated cells tuned to these spatial frequencies.) The cortical representation of at least one thin gray ring was completely filled in when surrounded by a low-spatial-frequency grating, but was clearly delimited when surrounded by a mid-to-high-spatialfrequency grating. These results solidly support the idea that (within a local cortical region) cells that are selectively responsive to a low-spatial-frequency grating have larger receptive fields, and that cells that are selectively responsive to a high-spatialfrequency grating have smaller receptive fields.

In the parafovea of this case, a comparison between DG and cytochrome oxidase staining patterns of the blankout rings showed that a low-spatial-frequency grating produces high DG uptake on and near the cytochrome oxidase blobs, and a highspatial-frequency grating produces uptake in the interblobs. Similar findings have been presented earlier (Tootell et al., 1982b). This correlates nicely with the demonstration that anatomical connections between blobs spread further than those between interblobs (Livingstone and Hubel, 1984b). The combined picture of these results is that cells with relatively low spatial frequency tuning (and larger receptive fields) are found in the blobs. They connect over a larger cortical area, as one might expect from cells with larger receptive fields. Conversely, cells tuned to higher spatial frequencies, with smaller receptive fields, are found in the interblob region and have connections that are locally more restricted.

\section{Variations with eccentricity}

It has been reported that the horizontal spread of physiological activity is greatest near the fovea, and least near $5^{\circ}$ eccentricity (Dow et al., 1981; Van Essen et al., 1984). When we examined the sharpness of the DG borders across this range, we found that they were in fact more blurred near the fovea relative to their resolution near $5^{\circ}$. This seems to support the electrophysiological evidence (Dow et al., 1981; Van Essen et al., 1984).

However, several complications arise that make this interpretation less straightforward. First, any residual eye movements during the period of DG uptake would blur DG borders much more near the fovea than peripherally, because a constant (small) shift in eye position is represented across a larger cortical region at the fovea. Thus, it could be argued that our apparent DG evidence for a decreased retinotopic resolution near the fovea is only an artifactual consequence of minor eye movements. There are 3 counterarguments to this particular concern. First, the blurring of DG patterns near the fovea was usually symmetric across the visual field (as eye drift is usually not). Second, artifacts due to eye movement were not seen in other retinotopic cases that received equal doses of paralytic (e.g., Fig. $20 B$ ). Third, the fact that we can see DG borders as sharp as those illustrated in Figures 18 and 19 indicates that eye movements are not a major worry.

A second complication arises when we are reminded that the DG evidence on variations of physiological spread with eccentricity actually differs quite a bit, depending on how that spread is measured. We used 2 different kinds of visual stimuli as measures of the physiological spread of activity. To serve as DG analogs of the cortical point image, we used small blinking checks on a uniform gray background, and to measure the minimum non-overlap distance we used thin gray rings surrounded by gratings of various sorts. Of these 2 types of stimuli, only the minimum overlap (blankout ring) case showed a striking shift with eccentricity; the eccentricity variations seen in the cortical point image (blinking checks) case were more modest.

Our interpretation of this discrepancy hinges on the differences between the 2 stimuli. The stimulus-driven DG activity in the blankout ring case was actually due to the grating surrounding the blankout ring. On the basis of our evidence, the minimum non-overlap distance increases closer to the fovea of the region stimulated with a 4.7 cycle/deg grating. However, a $4.7 \mathrm{cycle} / \mathrm{deg}$ grating produces highest uptake within the cytox blobs in the foveal representation (thus acting in this region as a relatively "low" spatial frequency), while it produces highest uptake in the interblob regions near and beyond the $5^{\circ}$ representation (thus acting as a "high" spatial frequency). Such a shift is obvious in comparisons of the effects of 1 cycle/deg ("low") and 4 cycle/deg ("high") spatial frequencies in the parafovea (scc Figs. 20,21). Thus, the apparcnt increase in the non-overlap measure may actually reflect the relative receptive-field extents of blob versus interblob neurons rather than an eccentricity variation per se. To resolve this issue in the DG studies, it will be necessary to stimulate a monkey similarly to that in Figure $20 \mathrm{~A}$, except with a higher-spatial-frequency grating as a background stimulus. However, the much smaller variation of DG falloff in the cortical point image case (Fig. 18) strongly suggests that the shift with eccentricity illustrated in Figures 20 and 22 is at least partially due to the spatial specificity of the background stimulus.

\section{Variations with lamina}

Since average receptive-field size appears to vary with lamina, the spread of physiological activity presumably also varies with lamina. Interpretation of the DG retinotopic resolution in the geniculostriate input layers is relatively straightforward. In both the minimum non-overlap and cortical point image cases, retinotopic resolution was sharpest in the parvocellular-recipient layer $4 \mathrm{Cb}$, and significantly more diffuse in the magnocellularrecipient layer 4Ca. Similar differences in horizontal spread between layers $4 \mathrm{Ca}$ and $4 \mathrm{Cb}$ were also seen in the counterphase grating casc (Fig. 23). Thesc DG diffcrences presumably directly reflect differences in the spread of afferent arborization in the 2 
layers (Blasdel and Lund, 1983). Resolution in 4A was about as sharp as it is in $4 \mathrm{Cb}$, and sharper than that in $4 \mathrm{Ca}$, which supports the idea that $4 \mathrm{~A}$ receives prominent input from the parvocellular LGN layers.

Intrinsic striate connections can also be rationalized with the DG data. For instance, in the point image case, the resolution in layer $4 \mathrm{~B}$ is approximately as diffuse as that in its major source layer $4 \mathrm{Ca}$, and more diffuse than that in $4 \mathrm{Cb}$, from which it does not receive major input.

In both the point image and the minimum non-overlap case, the retinotopic resolution in striate layer 3 is slightly degraded relative to that in primary parvorecipient layers $4 \mathrm{Cb}$ and $4 \mathrm{~A}$, but is significantly sharper than that in magnorccipient layer $4 \mathrm{Ca}$. Since the retinotopic resolution of the layer $3 \mathrm{DG}$ results is more discrete than that in $4 \mathrm{Ca}$ (but less than that in $4 \mathrm{Cb}$ and $4 \mathrm{~A}$ ), this supports the idea that the major input to layer 3 is derived from the parvocellular layers via $4 \mathrm{Cb}$ (or directly; Horton, 1984), rather than from the magnocellular layers via $4 \mathrm{Ca}$ (Blasdel et al., 1985; Fitzpatrick et al., 1985). Although an alternative pathway, from the intercalated LGN layers in the squirrel monkey, has been demonstrated to layer 3 (Fitzpatrick et al., 1983), one would guess this to be a retinotopically diffuse projection if it existed in the macaque because of the large receptive fields of the intercalated cells in other species (Irvin et al., 1986).

It is interesting to try to interpret the absence of activation in layers 2 and 6 of the point image case (actively stimulated ring; Fig. 18) in light of the results from corresponding layers of the minimum non-ovcrlap case (blankout ring; Fig. 23). In the point image case, the complete absence of activation in layers 2 and 6 indicates a nonlinear threshold for DG uptake in the cells projecting to layers 2 and 6, and/or in the cells of layers 2 and 6 themselves. However, such a nonlinearity could be exaggerated by a wide divergence in the retinotopic projection to layers 2 and 6 . Such a retinotopic divergence apparently occurs in layer 2 , in which the most obvious blankout ring of the highfrequency region is almost completely filled in (see Fig. 23). In layer 6 , the blankout ring is not filled in, indicating a fairly sharp retinotopic substrate in this layer. Thus, in layer 6 , the evidence for a thresholding mechanism is more compelling.

Other laminar differences are of note. In both the point image and minimum non-overlap case, the retinotopic DG patterns (indeed, almost all stimulus-specific DG patterns) differ between layers 2 and 3 . Unlike DG differences in other layers, the DG patterns in layers 2 and 3 change gradually rather than abruptly between these 2 layers. This supports the lack of distinct stratification between these 2 layers in numerous other anatomical studies.

In a number of previous studies (Fisken et al., 1975; Rockland and Lund, 1983; Blasdel et al., 1985), the intrinsic connections within layer 4B have been shown to be extremely wide-ranging, measuring up to $4 \mathrm{~mm}$. The falloff of DG uptake in the point image case in layer $4 \mathrm{~B}$ was only about $340 \mu \mathrm{m}$, and falloffs in the minimum non-overlap case were similar; both were well under the 1-2 $\mathrm{mm}$ that one might estimate as the half-spread of anatomically demonstrated connections in layer $4 \mathrm{~B}$. It is puzzling that such a robust set of anatomical connections would not be mirrored in a more diffuse retinotopic DG map. On the other hand, unusually large receptive fields or a disordered retinotopic map have not been reported in layer 4B single-unit data (Blasdel and Fitzpatrick, 1984; Livingstone and Hubel, 1984).

\section{Orientation-specific tests}

In one animal we presented a counterphased, square-wave grating of a very low spatial frequency. The stimulus was kept stationary in the visual field, and the edges of each grating stripe produced orientation-specific DG uptake. As in orientationspecific cases produced by moving gratings, DG orientation "columns" did not appear in parvocellular-recipient layers $4 \mathrm{Cb}$ and 4A, but did appear in magnocellular-recipient layer $4 \mathrm{Ca}$. The retinotopic spread of orientation-specific activity was generally greater in magnocellular-dominated layers (such as $4 \mathrm{~B}$ and $4 \mathrm{Ca}$ ) than in parvocellular-dominated layers (such as $4 \mathrm{~A}$ and $4 \mathrm{Cb}$ ). This increased spread of activity manifested itself as spotty regions of high DG uptake, including, and spreading beyond, the bounds of the stripe representation in adjacent $4 \mathrm{~A}$ and $4 \mathrm{Ca}$. In many places, the conclusion is inescapable that the spotty regions of high DG uptake in layer $4 \mathrm{Ca}$ extend into vertical registration with ocular dominance columns that are not obviously activated in layers $4 \mathrm{Cb}$ or in $4 \mathrm{~A}$. This supports other DG evidence for an extensive set of connections in layer $4 \mathrm{Ca}$ that extend beyond the bounds of a given ocular dominance strip (see Fig. 23 and Tootell et al., 1988a).

The relatively restricted spread of orientation-specific activity in layer 3 of this case is consistent with DG data from the point image and the minimum non-overlap case, which show a fairly sharp retinotopic resolution in layer 3 that is more restricted than that in layer $4 \mathrm{Ca}$ and less discrete than that in $4 \mathrm{Cb}$. Again, this presumably indicates that the $4 \mathrm{Cb}$ input to layer 3 is more prominent than that from $4 \mathrm{Ca}$.

The spread of activity in layer 6 of this case is quite extensive, compared to that in the minimum non-overlap case. Taken together, these 2 pieces of information may indicate that the long-range anatomical connections demonstrated in layer 6 (e.g., Blasdel et al., 1985) are involved in connecting cells of like orientation.

\section{Relationship of DG spread to electrophysiological spread}

Our initial impression of the DG maps was that they were retinotopically more discrete than one would expect from the electrophysiological data. However, when one examines the issue quantitatively, the discrepancy between the DG and electrophysiological measurements is quite small, perhaps within the range of measurement error. That is, the DG falloffs are about as sharp as one would expect from previous electrophysiological estimates of receptive-field size plus some straightforward linear assumptions about the relationship of DG uptake to extracellular receptive-field sensitivity.

We begin by estimating average receptive-field size and scatter. Both Dow et al. (1981) and Van Essen et al. (1984) report that cells at $3^{\circ}$ eccentricity have receptive fields with a mean size of about $20^{\prime}$ on a side. The absolute minimum and maximum receptive-field sizes at this eccentricity are unknown, but our estimates from their scatterplots are near $10^{\prime}$ and $40^{\prime}$ on a side, respectively. Below, we consider the additional contribution of retinotopic scatter to the blurring of DG uptake patterns.

Let us assume that the average receptive field has a sensitivity profile in which the envelope of absolute sensitivity (disregarding the sign of the response) is Gaussian in shape. By this approximation, the standard deviations of the minimum, mean, and maximum receptive-field sizes will be about $2.5,5$, and $10^{\prime}$ of arc ( $\mathrm{min}$ arc) on a side, respectively. Such a model receptive field will respond at half-maximum (or above) within a distance 
of $1.18 \pm \mathrm{SD}$ from the center (that is, within about 3,6 , and $12 \mathrm{~min}$ arc from the center, respectively).

For comparison, the half-amplitude of our cortical point image measurements ranges from about 140 to $500 \mu \mathrm{m}$ at $3^{\circ} \mathrm{ec}-$ centricity, depending on the layer. At this eccentricity, these cortical distances correspond to about $2.2-7.5 \mathrm{~min}$ arc. Ignoring the contribution of receptive-field scatter for a moment, the halfamplitude (in our examples, at half-width) of DG uptake can be compared most aptly with the half-maximum receptive-field sensitivity distances described above.

The half-amplitude of retinotopic uptake (2.2-7.5 min arc) is only slightly less than the receptive-field half-maxima (3-12 min arc). The addition of retinotopic scatter $\left(5.5 \mathrm{~min}\right.$ arc at $3^{\circ}$, according to Dow et al., 1981) to the receptive-field measurements of retinotopic disorder will tend to create a somewhat larger discrepancy between the 2 measures.

Thus, the DG borders are about twice as sharp as one might expect from the single-unit reports of receptive-field size. However, experimental considerations work to minimize that discrepancy. First, in electrophysiological studies there is always the possibility that eye movements have significantly inflated the measurements of retinotopic disorganization; in more rapid DG mapping experiments, the possibility of eye movements is much less worrisome, and easier to spot if it occurs. Second, in our DG tests we often used rays and rings with sharp borders. Thus, the DG borders might selectively reflect the activity of cells with smaller-than-average (higher-spatial-frequency), rather than average-sized, receptive fields.

The match between the DG border falloffs and singlc-unit receptive-field measurements is thus fairly good. A priori, it is not clear that the match would necessarily have been so close. For one thing, the DG and electrophysiological data may reflect activity in different cellular compartments. The DG may reflect dendritic and synaptic activity that might not appear at the level of the somal action potential, recorded electrophysiologically. It is also by no means clear that a linear increase in single-unit activity will produce a correspondingly linear increase in DG uptake. Such considerations might yet need to be invoked in order to explain fine-grained differences between DG and singleunit data in future studies. If we assume that the DG border falloffs are different enough from the single-unit reports that an explanation is called for, one logical extension of these ideas is that the gain of DG uptake with increasing activation is steeper than the corresponding gain for extracellularly measured spikes per second, or that there is a higher threshold for DG uptake than for spike activity.

\section{Retinotopic results and acuity limits}

In a quantitative comparison of DG falloffs and acuity limits, we concluded that the half-amplitude of the DG borders in striate layer $4 \mathrm{Cb}$ are approximately equal to the classical acuity limits. In fact, however, the actual retinotopic borders may be even sharper than they appear in our measurements of the DG falloffs. To begin with, the bandwidth of the point spread function (full-width at half-amplitude) of the ${ }^{14} \mathrm{C}$ autoradiographic method itself is about $100 \mu \mathrm{m}$ (Goochee et al., 1980). The DG borders that were measured are therefore the actual DG uptake convoluted by a Gaussian of standard deviation $\sim 42 \mu \mathrm{m}$. Other experimental factors (such as histological diffusion, eye drift during DG uptake, and the use of large-grain $x$-ray film) would also artifactually increase the measured retinotopic falloffs. Considering all these known and possible artifacts, it is clear that the values we have obtained are confounded with the resolution limitations of the DG measurement technique to some extent. The true falloffs must be, if anything, steeper. It is thus possible that striate retinotopic organization is even more discrete than we have given it experimental credit for.

Purely as an exercise, it is interesting to compare the DG falloffs and the hyperacuity limits carefully. A simple way to shore up the evidence for hyperacuity resolution in layer $4 \mathrm{Cb}$ is to suppose that differences in levels of uptake that are smaller than half the total range of stimulus-driven uptake are still adequate to account for hyperacuity. Specifically, the hyperacuity threshold at $3^{\circ}$ eccentricity corresponds to a horizontal spread of $-27 \mu \mathrm{m}$ on the particular cortex we examined in Figure 23. At the steepest portion of the DG falloffs, $27 \mu \mathrm{m}$ on the cortex corresponds to a $5 \%$ difference in DG uptake, relative to baseline levels. On the face of it, a 5\% difference in DG uptake appears adequate to explain psychophysical thresholds based on extensive training and threshold criteria of only $75 \%$ correct.

\section{Physiological versus anatomical spread and the "module" concept}

Our analysis of physiological spread shows it to be fairly complicated. The minimum non-overlap distance/cortical point image varies as a function of (1) the layer in which the activity is sampled, (2) the size of the sampled receptive fields, (3) the orientation of the sampled receptive fields, and, probably, (4) the eccentricity at which the activity is sampled. In other experiments, it also appears that the physiological spread of activity varies as a function of chromatic variables (unpublished observations). As a result of all these factors, the physiological spread we have seen in different DG tests ranges from about $140 \mu \mathrm{m}$ to more than a millimeter, when specified in terms of the half-width at half-amplitude of DG falloff. There is clearly no single measure of physiological spread that can be used without a long list of qualifiers.

Van Essen et al. (1984) have pointed out that the blobs and hypercolumns furnish 2 "conceptually distinct bases for defining modules," in addition to measurements of physical spread. This naturally begs the question, "What is a module?" Presumably, the free evolution of scientific jargon will eventually settle on a "true" definition of the striate cortical module. Until then, we may need to content ourselves with the provisional notion of 2 kinds of striate cortical "module." One is a physiological module with dynamic functional boundaries, which cannot be defined without a long list of functional qualifiers. The blobs furnish an alternative, anatomically based definition of the cortical module. One obvious advantage of the anatomical "module" is, of course, that it can be directly demonstrated using appropriate histological techniques. It may be that the denouement of the whole controversy hinges on the ease with which the competing concepts can be taught to undergraduates and medical students; in this case, the fact that the anatomical modules can be directly photographed is a telling point. Another advantage of the cytox blob module is that it appears intimately related to the spacing and geometry of the various hypercolumns (Horton and Hubel, 1981; Tootell et al., 1982a, b; Horton, 1984), whereas the measurements of physical spread may vary in size with eccentricity (Van Essen et al., 1984).

One of the ways in which anatomical and physiological measurements of spread can be directly related is by determining the size of the visual field corresponding to the distance between adjacent cytox blobs. At the foveal representation, the distance 
between the centers of adjacent cytox blobs corresponds to a visual angle of about $4^{\prime}$ of arc. At $8^{\circ}$ eccentricity, this distance increases to about $1 / 2^{\circ}$.

One of the main reasons for studying vision in the macaque is that its visual system is so closely related to that of man, and in the present context we wanted to know how large a visualfield region was served by a blob domain in the human. In another study (unpublished observations), human striate cortex was unfolded and the blobs counted in a few hemispheres. Although there is some ambiguity in separating blobs in the human, we estimated about 3000 blobs in human striate cortex. This total is significantly smaller than the estimates of Horton and Hedley-Whyte (1984), who suggest that human striate cortex may contain about as many blobs as are found in the macaque. Much of the discrepancy can be traced to differences in estimates of human striate cortex. We adopted the estimate of Stensaas et al. (1974) of $21.3 \mathrm{~cm}^{2}$, which was based on measurements of 52 hemispheres. Horton and Hedley-Whyte (1984) favored a higher estimate of $30-36 \mathrm{~cm}^{2}$. By our estimates, the distance between human cytox blobs would correspond to about $10^{\prime}$ of arc at the foveal representation. At other eccentricities, the corresponding size of the visual-field representation in human striate cortex can be approximated by multiplying the $\mathrm{Y}$-axis values in Figure 25 by about 2.5 .

\section{References}

Blasdel. G. G., and D. Fitzpatrick (1984) Physiological organization of layer 4 in macaque striate cortex. J. Neurosci. 4: 880-895.

Blasdel, G. G., and J. S. Lund (1983) Termination of afferent axons in macaque striate cortex. J. Neurosci. 3: 1389-1413.

Blasdel, G. G., J. S. Lund, and D. Fitzpatrick (1985) Intrinsic connections of macaque striate cortex: Axonal projections of cells outside lamina 4C. J. Neurosci. 5: 3350-3369.

Borwein, B., D. Borwein, J. Medeiros, and J. W. McGowan (1980) The ultrastructure of monkey fovea photoreceptors, with special reference to the structure, shape, size and spacing of foveal cones. Am. J. Anat. 159: 125-146.

Bough, E. W. (1970) Stereoscopic vision in the macaque monkey: A behavioral demonstration. Nature 225: 42-44.

Bunt, A. H., D. S. Minckler, and G. H. Johansen (1977) Demonstration of a bilateral projection of the central retina of the monkey with horseradish peroxidase neuronography. J. Comp. Neurol. 171:619630.

Burkhalter, A., D. J. Felleman, W. T. Newsome, and D. C. Van Essen (1986) Anatomical and physiological asymmetries related to visual areas V3 and VP in macaque extrastriate cortex. Vision Res. 26: 6380.

Cowey, A., and C. M. Ellis (1967) Visual acuity of rhesus and squirrel monkcy. J. Comp. Physiol. Psychol. 64: 80-84.

Cusick, C. G., H. J. Gould III, and J. H. Kaas (1984) Interhemispheric connections of visual cortex of owl monkey (Aotus trivirgatus), marmosets (Callithrix jaccus) and galagos (Galago cressicaudatus). J. Comp. Neurol. 230: 311-336.

Daniel, P. M., and D. Whitteridge (1961) The representation of the visual field on the cerebral cortex in monkeys. J. Physiol. 159: 203221.

De Valois, R. I., H. C. Morgan, M. C. Polson, W. R. Mead, and E. M. Hull (1974a) Psychophysical studies on monkey vision I. Macaque luminosity and color tests. Vision Res. 14: 53-67.

De Valois, R. L., H. C. Morgan, and D. M. Snodderly (1974b) Psychophysical studies of monkey vision III. Spatial contrast sensitivity tests of macaque and human observers. Vision Res. 14: 751-781.

De Valois, R. L., D. G. Albrecht, and L. G. Thorell (1982) Spatial frequency selectivity of cells in macaque visual cortex. Vision Res. 22: 545-559.

Dow, B., and P. Gouras (1973) Color and spatial specificity of single units in rhesus monkey foveal striate cortex. J. Neurophysiol. 36: 79100.

Dow, B. M., A. Z. Snyder, R. G. Vautin, and R. Bauer (1981) Mag- nification factor and receptive field size in foveal striate cortex of monkey. Exp. Brain Res. 44: 213-288.

Dow, B. M., A. Z. Snyder, R. G. Vautin, and R. Bauer (1985) The mapping of visual space onto foveal striate cortex in the macaque monkey. J. Neurosci. 5: 890-902.

Felleman, D. J., G. J. Carman, and D. C. Van Essen (1984) Evidence for a functional distinction between areas V3 and VP of macaque extrastriate cortex. Invest. Opththalmol. Vis. Sci. (Suppl.) 25: 278.

Fisken, R. A., L. J. Garey, and T. P. S. Powell (1975) The intrinsic association and commissural connections of area 17 of the visual cortex. Phil. Trans. R. Soc. Lond. [Biol.]272: 487-536.

Fitzpatrick, D., K. Itoh, and I. T. Diamond (1983) The laminar organization of the lateral geniculate body and the striate cortex in squirrel monkey (Saimiri sciureus). J. Neurosci. 3: 673-702.

Fitzpatrick, D., J. S. Lund, and G. G. Blasdel (1985) Intrinsic connections of macaque striate cortex: Afferent and efferent connections of lamina 4C. J. Neurosci. 5: 3329-3349.

Gilbert, C. D. (1977) Laminar differences in receptive field properties of cells in cat primary visual cortex. J. Physiol. (Lond.) 268: 391421.

Goochee, C., W. Rasband, and L. Sokoloff (1980) Computerized densitometry and color coding of $\left[{ }^{14} \mathrm{C}\right]$ deoxyglucose autoradiographs. Ann. Neurol. 7: 359-370.

Granger, E. M., and J. C. Heurtley (1973) Visual chromaticity modulation transfer function. J. Opt. Soc. Am. 63: 1173-1174.

Guld, C., and A. Bertulis (1976) Representation of fovea in the striate cortex of vervet monkey, Cercopithecus aethiops pygerythrus. Vision Res. 16: 629-631.

Horton, J. C. (1984) Cytochrome oxidase patches: A new cytoarchitectonic feature of monkey cortex. Phil. Trans. R. Soc. Lond. [Biol.] 304: 199-253

Horton, J. C., and E. T. Hedley-Whyte (1984) Mapping of cytochrome oxidase patches and ocular dominance columns in human visual cortex. Phil. Trans. R. Soc. Lond. [Biol.] 304: 255-272.

Horton, J. C., and D. H. Hubel (1981) Regular patchy distribution of cytochrome oxidase staining in primary visual cortex of the macaque monkey. Nature 292: 762-764.

Hubel, $D$. $H$., and T. N. Wiesel (1968) Receptive fields and functional architecture of monkey striate cortex. J. Physiol. (Lond) 195: 215243.

Hubel, D. H., and T. N. Wiesel (1974) Sequence regularity and gcometry of orientation columns in monkey striate cortex. J. Comp. Neurol. 158: 267-294.

Hubel, D. H., and T. N. Wiesel (1977) Functional architecture of macaque visual cortex. Ferrier lecture. Proc. R. Soc. Lond. [Biol.] 198: $1-59$.

Hubel, D. H., T. N. Wiesel, and S. LeVay (1974) Visual field of representation in layer IVC of monkey striate cortex. Soc. Neurosci. Abstr. 1974: 264.

Hubel, D. H., T. N. Wiesel, and M. P. Stryker (1978) Anatomical demonstration of orientation columns in macaque monkey. J. Comp. Neurol. 177: 361-380.

Irvin, G. E., T. T. Norton, M. A. Sesma, and V. A. Casagrande (1986) W-like response properties of interlaminar zone cells in the lateral geniculate nucleus of a primate. Brain Res. 362: 254-270.

Kirk, D. L., W. R. Levick, B. G. Cleland, and H. Wassle (1976a) Crossed and uncrossed representation of the visual field by brisksustained and brisk-transient cat retinal ganglion cells. Vision Res. 16: $225-231$.

Kirk, D. L., W. R. Levick, and B. G. Cleland (1976b) The crossed or uncrossed destination of axons of sluggish-concentric and non-concentric cat retinal ganglion cells, with an overall synthesis of the visual field representation. Vision Res. 16: 233-236.

LeVay, S. D., D. H. Hubel, and T. N. Wiesel (1975) The pattern of ocular dominance in macaque visual cortex revealed by a reduced silver stain. J. Comp. Neurol. 159: 559-576.

LeVay, S., M. Connally, J. Houde, and D. C. Van Essen (1985) The complete patterns of ocular dominance stripes in the striate cortex and visual field of the macaque monkey. J. Neurosci. 5: 486-501.

Livingstone, M. S., and D. H. Hubel (1984a) Anatomy and physiology of a color system in the primate visual cortex. J. Neurosci. 4: 309356.

Livingstone, M. S., and D. H. Hubel (1984b) Specificity of intrinsic connections in primary visual cortex. J. Neurosci. 4: 2830-2835.

Lund, J. S., and R. G. Boothe (1975) Interlaminar connections and 
pyramidal neuron organization in the visual cortex, area 17 , of the macaque monkey. J. Comp. Neurol. 159: 305-334.

McCourt, M. E. (1982) A spatial frequency dependent grating induction effect. Vision Res. 22: 119-134.

Mcllwain, J. T. (1986) Point images in the visual system: New interest in an old idea. Trends Neurosci. 9: 354-358.

Merker, B., and E. Schwartz (1985) Computer aided anatomy: Reconstruction and characterization of the opercular surface of macaque striate cortex. Investig. Ophthalmol. Vis. Sci. (Suppl.) 26:164.

Michael, C. R. (1981) Columnar organization of color cells in monkey's striate cortex. J. Neurophysiol. 46: 587-604.

Movshon, J. A., I. D. Thompson and D. J. Tolhurst (1978) Spatial and temporal contrast sensitivity of neurones in area 17 and 18 of the cat's visual cortex. J. Physiol. (Lond). 283: 101-120.

Palmer, L. A., and A. C. Rosenquist (1974) Visual receptive fields of single striate cortical units projecting to the superior colliculus in the cat. Brain Res. 67: 27-42.

Perry, V. H., and A. Cowey (1985) The gangion cell and cone distributions in the monkey retina: Implications for central magnification factors. Vision Res. 25: 1795-1810.

Perry, V. H., R. Oehler, and A. Cowey (1984) Retinal ganglion cells that project to the dorsal lateral geniculate nucleus in the macaque monkey. Neuroscience 12: 1101-1123.

Rockland, K. S., and J. S. Lund (1983) Intrinsic laminar lattice connections in primate visual cortex. J. Comp. Neurol. 216: 303-318.

Sakitt, B. (1982) Why the cortical magnification factor in rhesus cannot be isotropic. Vision Res. 22: 417-421.

Sarmiento, R. (1975) The stereoacuity of macaque monkey. Vision Res. 15: 493-498.

Schwartz, E. L. (1980) Computational anatomy and functional architecture of striate cortex: A spatial mapping approach to perceptual coding. Vision Res. 20: 645-669.

Schwartz, E. L. (1985) On the mathematical structure of the visuotopic mapping of macaque striate cortex. Science 227: 1065-1066.

Silverman, M. S. (1984) Deoxyglucose and electrophysiological evidence for spatial frequency columns in cat striate cortex. Ph.D. thesis, University of California, San Francisco.

Stensaas, S. S., D. K. Eddington, and W. H. Dobelle (1974) The topography and variability of the primary visual cortex in man. J. Neurosurg. 40: 747-755.

Stone, J., L. Leicester, and S. M. Sherman (1973) The naso-temporal division of the monkey's retina. J. Comp. Neurol. 150: 333-348.

Talbot, S. A., and W. H. Marshall (1941) Physiological studies on neural mechanisms of visual localization and discrimination. Am. J. Opththalmol. 74: 1255-1264.

Tootell, R. B. H., and G. G. Blasdel (1987) In vivo demonstration of presumptive blob and interblob regions by manipulation of stimulus spatial frequency in macaque striate cortex. Soc. Neurosci. Abstr. 13: 2.

Tootell, R. B. H., and M. S. Silverman (1981) A comparison of cytochrome oxidase and deoxyglucose patterns in macaque visual cortex. Soc. Neurosci. Abstr. 7: 356.

Tootell, R. B. H., and M. S. Silverman (1985) Two methods for flatmounting cortical tissue. J. Neurosci. Methods 15: 177-190.
Tootell, R. B. H., M. S. Silverman, and R. L. De Valois (1981) Spatial frequency columns in primary visual cortex. Science 214: 813-815.

Tootell, R. B. H., M. S. Silverman, E. Switkes, and R. L. De Valois (1982a) Deoxyglucose analysis of retinotopic organization in primate striate cortex. Science 218: 902-904.

Tootell, R. B. H., M. S. Silverman, E. Switkes, and R. L. DeValois (1982h) Organization of cortical modules. Soc. Neurosci. Abstr. 8. 707.

Tootell, R. B. H., M. S. Silverman, R. L. De Valois and G. H. Jacobs (1983a) Functional organization of the second visual area of primates. Science 220: 737-739.

Tootell, R. B. H., M. S. Silverman, R. L. De Valois, and G. H. Jacobs (1983b) Functional anatomy of primate V2. Invest. Ophthalmol. Vis. Sci. (Suppl.) 24; 106.

Tootell, R. B. H., S. L. Hamilton, M. S. Silverman, and E. Switkes (1988a) Functional anatomy of macaque striate cortex. I. Ocular dominance, binocular interactions, and baseline conditions. J. Neurosci. 8: 1500-1530.

Tootell, R. B. H., M. S. Silverman, E. L. Hamilton, R. L. De Valois, and E. Switkes (1988b) Functional anatomy of macaque striate cortex. III. Color. J. Neurosci. 8: 1569-1593.

Tootell, R. B. H., S. L. Hamilton, and E. Switkes (1988c) Functional anatomy of macaque striate cortex. IV. Contrast and magno-parvo streams. J. Neurosci. 8: 1594-1609.

Tootell, R. B. H., M. S. Silverman, S. L. Hamilton, E. Switkes, and R. L. De Valois (1988d) Functional anatomy of macaque striate cortex. V. Spatial frequency. J. Neurosci. 8: 1610-1624.

T'so, D., C. Gilbert, and T. N. Wiesel (1983) Horizontal connections in striate cortex as revealed by cross-correlation analysis. Soc. Neurosci. Abstr. 9: 476.

Tusa, R. J., L. A. Palmer, and A. C. Rosenquist (1978) The retinotopic organization of area 17 (striate cortex) in the cat. J. Comp. Neurol. 177: 213-236.

Van Essen, D. C., and J. H. R. Maunsell (1980) Two-dimensional maps of the cerebral cortex. I. Comp. Neurol. 191: 255-281.

Van Essen, D. C., and J. H. R. Maunsell (1983) Hierarchical organization and functional streams in the visual cortex. Trends Neurosci. 6: 370-374.

Van Essen, D. C., W. T. Newsome, and J. H. R. Maunsell (1984) The visual field representation in striate cortex of the macaque monkey: Asymmetries, anisotropies and individual variability. Vision Res. 24: 429-448.

Watanabe, A., H. Sakata, and H. Isono (1976) Chromatic spatial sine wave response of the human visual system. NHK Lab Note 198: 110.

Westheimer G. (1979) The spatial sense of the eye. Invest. Ophthalmol. Vis. Sci. 18: 893-912.

Westheimer, G. (1982) The spatial grain of the perifoveal visual field. Vision Res. 22: 157-162.

Whitteridge, D. (1965) Geometrical relations between the retina and the visual cortex. In Mathematics and Computer Science in Biology and Medicine, Medical Research Council, London. 